\title{
Synthesis of 2-Imino-1,3,4-thiadiazoles from
}

\section{Hydrazides and Isothiocyanates via Sequential Oxidation and $\mathrm{P}\left(\mathrm{NMe}_{2}\right)_{3}$-Mediated Annulation Reactions}

Zhengyan Huang, Qianqian Zhang, Qiongli Zhao, Wenquan Yu* and Junbiao Chang* College of Chemistry, Zhengzhou University, Zhengzhou, Henan Province 450001, China.

\section{CONTENTS:}

1. General Information

2. General Procedure for the Synthesis of Products 4

3. References

4. NMR Spectra of Compounds 4 
1. General Information. ${ }^{1} \mathrm{H}$ and ${ }^{13} \mathrm{C}$ NMR spectra were recorded on a $400 \mathrm{MHz}(100$ $\mathrm{MHz}$ for ${ }^{13} \mathrm{C}$ NMR) spectrometer. Chemical shift values are reported in ppm (parts per million) with tetramethylsilane (TMS) as an internal standard. The peak patterns are indicated as follows: s, singlet; d, doublet; t, triplet; $\mathrm{q}$, quartet; hept, heptet; m, multiplet; dd, doublet of doublets; dt, doublet of triplets; td, triplet of doublets; tt, triplet of triplets. The coupling constants $(J)$ are reported in hertz $(\mathrm{Hz})$. Melting points were determined on a micromelting point apparatus and are uncorrected. High-resolution mass spectra (HRMS) were determined on a Q-TOF Mass Spectrometer equipped with an electrospray ion source (ESI), operated in the positive mode. Flash column chromatography was performed over silica gel 200-300 mesh and the eluents were distilled prior to use. Solvents for the synthesis of compounds 2 and 4 were analytical reagent grade and used without any pretreatment.

2. General Procedure for the Synthesis of Products 4. A solution of the hydazide (1, $0.5 \mathrm{mmol})$ in $\mathrm{MeCN}(5 \mathrm{~mL})$ was treated sequentially with $\mathrm{I}_{2}(0.6 \mathrm{mmol})$ and $\mathrm{K}_{2} \mathrm{CO}_{3}$ (1.5 mmol), and then stirred at room temperature $\left(25^{\circ} \mathrm{C}\right)$ for $0.5 \mathrm{~h}(1 \mathrm{~h}$ for $\mathbf{1 p}, \mathbf{1 q}$, and 1ad) until TLC indicated the disappearance of $\mathbf{1}$. The reaction was subsequently quenched with sat. $\mathrm{Na}_{2} \mathrm{~S}_{2} \mathrm{O}_{3}(4 \mathrm{~mL})$, diluted with $\mathrm{H}_{2} \mathrm{O}(20 \mathrm{~mL})$, and extracted with EtOAc $(10 \mathrm{~mL} \times 3)$. The combined organic layer was dried over anhydrous $\mathrm{Na}_{2} \mathrm{SO}_{4}$ and concentrated in vacuo to give the crude $N$-acyldiazene (2).

A mixture of the corresponding isothiocyanate $(3,1 \mathrm{mmol})$ and $\mathrm{P}\left(\mathrm{NMe}_{2}\right)_{3}(1.1$ mmol) in toluene $(6 \mathrm{~mL})$ was stirred at $20^{\circ} \mathrm{C}$ for $0.5 \mathrm{~h}$, and then treated with a solution of the above crude $N$-acyldiazene (2) in toluene $(4 \mathrm{~mL})$. Upon the completion of the reaction at $20^{\circ} \mathrm{C}$ when TLC indicated the disappearance of the $\mathrm{N}$-acyldiazene, the reaction was quenched with $\mathrm{H}_{2} \mathrm{O}(20 \mathrm{~mL})$ and extracted with EtOAc $(10 \mathrm{~mL} \times 3)$. 
The combined organic layer was dried over anhydrous $\mathrm{Na}_{2} \mathrm{SO}_{4}$, concentrated, and then purified through silica gel column chromatography to afford the 2-imino-1,3,4-thiadiazole product (4).

(Z)-N,3,5-Triphenyl-1,3,4-thiadiazol-2(3H)-imine (4a). 1 h; eluent: EtOAc/PE 1:300; yield: $110 \mathrm{mg}, 67 \%$ (0.5 mmol scale); yield: $1.14 \mathrm{~g}, 69 \%$ (5 mmol scale); white solid, mp 115-117 ${ }^{\circ} \mathrm{C}$ (lit. $\left..{ }^{1} \mathrm{mp} 113-116{ }^{\circ} \mathrm{C}\right) ;{ }^{1} \mathrm{H}$ NMR (400 MHz, $\left.\mathrm{CDCl}_{3}\right) 8.11$ (d, $J=7.6$ $\mathrm{Hz}, 2 \mathrm{H}), 7.70-7.68(\mathrm{~m}, 2 \mathrm{H}), 7.48-7.44(\mathrm{~m}, 2 \mathrm{H}), 7.43-7.36(\mathrm{~m}, 5 \mathrm{H}), 7.27(\mathrm{t}, J=7.6$

$\mathrm{Hz}, 1 \mathrm{H}), 7.14-7.08(\mathrm{~m}, 3 \mathrm{H}) ;{ }^{13} \mathrm{C} \mathrm{NMR}\left(100 \mathrm{MHz}, \mathrm{CDCl}_{3}\right) \delta 155.4,152.4,146.5$, $139.8,130.5,130.4,129.6,128.9,128.7,126.1,126.0,124.0,122.2$, 120.9; HRMS (ESI-TOF) $\mathrm{m} / \mathrm{z}$ : $[\mathrm{M}+\mathrm{H}]^{+}$calcd for $\mathrm{C}_{20} \mathrm{H}_{16} \mathrm{~N}_{3} \mathrm{~S} 330.1059$, found 330.1062 .

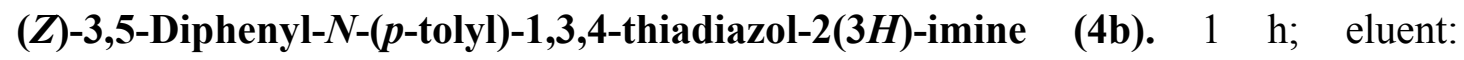
EtOAc/PE 1:300; yield: $110 \mathrm{mg}, 64 \%$; white solid, mp $114-115{ }^{\circ} \mathrm{C} ;{ }^{1} \mathrm{H}$ NMR (400 $\left.\mathrm{MHz} \mathrm{CDCl}_{3}\right) \delta 8.11(\mathrm{~d}, J=8.0 \mathrm{~Hz}, 2 \mathrm{H}), 7.70-7.68(\mathrm{~m}, 2 \mathrm{H}), 7.48-7.40(\mathrm{~m}, 5 \mathrm{H}), 7.26$ $(\mathrm{t}, J=7.6 \mathrm{~Hz}, 1 \mathrm{H}), 7.18(\mathrm{~d}, J=8.0 \mathrm{~Hz}, 2 \mathrm{H}), 6.99(\mathrm{~d}, J=6.8 \mathrm{~Hz}, 2 \mathrm{H}), 2.35(\mathrm{~s}, 3 \mathrm{H}) ;{ }^{13} \mathrm{C}$ NMR $\left(100 \mathrm{MHz}, \mathrm{CDCl}_{3}\right) \delta 155.2,150.0,146.4,139.8,133.4,130.5,130.4,130.2$, 128.9, 128.7, 126.1, 125.9, 122.2, 120.7, 21.0; HRMS (ESI-TOF) m/z: $[\mathrm{M}+\mathrm{H}]^{+}$calcd for $\mathrm{C}_{21} \mathrm{H}_{18} \mathrm{~N}_{3} \mathrm{~S}$ 344.1216, found 344.1215.

(Z)- $N$-(4-Methoxyphenyl)-3,5-diphenyl-1,3,4-thiadiazol-2(3H)-imine (4c). $1.5 \mathrm{~h}$; eluent: EtOAc/PE 1:200; yield: $80 \mathrm{mg}$, 44\%; white solid, mp $129-130{ }^{\circ} \mathrm{C}$; ${ }^{1} \mathrm{H}$ NMR $\left(400 \mathrm{MHz}, \mathrm{CDCl}_{3}\right) \delta 8.10(\mathrm{dd}, J=8.8,0.8 \mathrm{~Hz}, 2 \mathrm{H}), 7.71-7.68(\mathrm{~m}, 2 \mathrm{H}), 7.48-7.44(\mathrm{~m}$, 2H), 7.43-7.39 (m, 3H), $7.26(\mathrm{t}, J=7.6 \mathrm{~Hz}, 1 \mathrm{H}), 7.05-7.01(\mathrm{~m}, 2 \mathrm{H}), 6.94-6.90(\mathrm{~m}$, 2H), $3.81(\mathrm{~s}, 3 \mathrm{H}) ;{ }^{13} \mathrm{C} \mathrm{NMR}\left(100 \mathrm{MHz}, \mathrm{CDCl}_{3}\right) \delta 156.2,155.4,146.3,145.9,139.9$, 130.5, 130.4, 128.9, 128.7, 126.1, 125.9, 122.2, 121.8, 114.8, 55.5; HRMS (ESI-TOF) $\mathrm{m} / \mathrm{z}:[\mathrm{M}+\mathrm{H}]^{+}$calcd for $\mathrm{C}_{21} \mathrm{H}_{18} \mathrm{~N}_{3} \mathrm{OS} 360.1165$, found 360.1167 .

(Z)-N-(4-Chlorophenyl)-3,5-diphenyl-1,3,4-thiadiazol-2(3H)-imine (4d). 1 h; eluent: EtOAc/PE 1:400; yield: $135 \mathrm{mg}, 74 \%$; white solid, mp 131-133 ${ }^{\circ} \mathrm{C} ;{ }^{1} \mathrm{H}$ NMR (400 $\left.\mathrm{MHz}, \mathrm{CDCl}_{3}\right) \delta 8.06(\mathrm{~d}, J=8.0 \mathrm{~Hz}, 2 \mathrm{H}), 7.71-7.68(\mathrm{~m}, 2 \mathrm{H}), 7.49-7.40(\mathrm{~m}, 5 \mathrm{H})$, 7.34-7.27 (m, 3H), $7.02(\mathrm{~d}, J=8.4 \mathrm{~Hz}, 2 \mathrm{H}) ;{ }^{13} \mathrm{C} \mathrm{NMR}\left(100 \mathrm{MHz}, \mathrm{CDCl}_{3}\right) \delta 155.9$, $150.9,146.6,139.6,130.6,130.3,129.7,129.02$, 128.97, 128.8, 126.3, 126.2, 122.44, 
122.40; HRMS (ESI-TOF) m/z: [M + H] ${ }^{+}$calcd for $\mathrm{C}_{20} \mathrm{H}_{15} \mathrm{ClN}_{3} \mathrm{~S} 364.0670$, found 364.0669 .

(Z)-3,5-Diphenyl- $N$-(4-(trifluoromethyl)phenyl)-1,3,4-thiadiazol-2(3H)-imine (4e). $1 \mathrm{~h}$; eluent: EtOAc/PE 1:250; yield: $168 \mathrm{mg}, 85 \%$; white solid, mp $146-148{ }^{\circ} \mathrm{C} ;{ }^{1} \mathrm{H}$ NMR (400 MHz, $\left.\mathrm{CDCl}_{3}\right) \delta 8.07(\mathrm{~d}, J=8.4 \mathrm{~Hz}, 2 \mathrm{H}), 7.73-7.70(\mathrm{~m}, 2 \mathrm{H}), 7.63(\mathrm{~d}, J=$ $8.4 \mathrm{~Hz}, 2 \mathrm{H}), 7.51-7.42(\mathrm{~m}, 5 \mathrm{H}), 7.32(\mathrm{t}, J=7.6 \mathrm{~Hz}, 1 \mathrm{H}), 7.18(\mathrm{~d}, J=8.4 \mathrm{~Hz}, 2 \mathrm{H}) ;{ }^{13} \mathrm{C}$ NMR $\left(100 \mathrm{MHz}, \mathrm{CDCl}_{3}\right) \delta 156.0,155.1,146.8,139.5,130.7,130.2,129.0,128.8$, $126.9\left(\mathrm{q}, J_{\mathrm{C}-\mathrm{F}}=3.8 \mathrm{~Hz}\right), 126.5,126.2,125.7\left(\mathrm{q}, J_{\mathrm{C}-\mathrm{F}}=32.4 \mathrm{~Hz}\right), 124.4\left(\mathrm{q}, J_{\mathrm{C}-\mathrm{F}}=270.0\right.$ $\mathrm{Hz}$ ), 122.6, 121.3; HRMS (ESI-TOF) $\mathrm{m} / \mathrm{z}:[\mathrm{M}+\mathrm{H}]^{+}$calcd for $\mathrm{C}_{21} \mathrm{H}_{15} \mathrm{~F}_{3} \mathrm{~N}_{3} \mathrm{~S} 398.0933$, found 398.0931 .

(Z)-4-((3,5-Diphenyl-1,3,4-thiadiazol-2(3H)-ylidene)amino)benzonitrile (4f). $1 \mathrm{~h}$, eluent: EtOAc/PE 1:50; yield: $156 \mathrm{mg}, 88 \%$; white solid, mp $145-147{ }^{\circ} \mathrm{C}$; ${ }^{1} \mathrm{H}$ NMR $\left(400 \mathrm{MHz}, \mathrm{CDCl}_{3}\right) \delta 8.02(\mathrm{~d}, J=7.6 \mathrm{~Hz}, 2 \mathrm{H}), 7.71-7.69$ (m, 2H), 7.64 (d, $J=8.4 \mathrm{~Hz}$, 2H), 7.50-7.42 (m, 5H), $7.31(\mathrm{t}, J=7.6 \mathrm{~Hz}, 1 \mathrm{H}), 7.15(\mathrm{~d}, J=8.4 \mathrm{~Hz}, 2 \mathrm{H}) ;{ }^{13} \mathrm{C} \mathrm{NMR}$ $\left(100 \mathrm{MHz}, \mathrm{CDCl}_{3}\right) \delta 156.1,155.7,147.0,139.2,133.8,130.9,129.9,129.0,128.8$, 126.7, 126.2, 122.7, 121.8, 119.3, 106.7; HRMS (ESI-TOF) m/z: $[\mathrm{M}+\mathrm{H}]^{+}$calcd for $\mathrm{C}_{21} \mathrm{H}_{15} \mathrm{~N}_{4} \mathrm{~S}$ 355.1012, found 355.1013.

(Z)-N-(4-Nitrophenyl)-3,5-diphenyl-1,3,4-thiadiazol-2(3H)-imine (4g). ${ }^{2} 1 \mathrm{~h}$, eluent: $\mathrm{CH}_{2} \mathrm{Cl}_{2} / \mathrm{PE} \mathrm{1:4;} \mathrm{yield:} 180 \mathrm{mg}, 96 \%$; yellow solid, mp $181-183{ }^{\circ} \mathrm{C}$; ${ }^{1} \mathrm{H}$ NMR (400 $\left.\mathrm{MHz}, \mathrm{CDCl}_{3}\right) \delta 8.27-8.24(\mathrm{~m}, 2 \mathrm{H}), 8.04-8.01(\mathrm{~m}, 2 \mathrm{H}), 7.73-7.71(\mathrm{~m}, 2 \mathrm{H}), 7.52-7.44$ $(\mathrm{m}, 5 \mathrm{H}), 7.34(\mathrm{t}, J=7.6 \mathrm{~Hz}, 1 \mathrm{H}), 7.21-7.18(\mathrm{~m}, 2 \mathrm{H}) ;{ }^{13} \mathrm{C} \mathrm{NMR}\left(100 \mathrm{MHz}, \mathrm{CDCl}_{3}\right) \delta$ $157.6,156.3,147.2,143.5,139.1,131.0,129.8,129.1,128.9,126.9,126.2,125.7$, 122.9, 121.4; HRMS (ESI-TOF) m/z: $[\mathrm{M}+\mathrm{H}]^{+}$calcd for $\mathrm{C}_{20} \mathrm{H}_{15} \mathrm{~N}_{4} \mathrm{O}_{2} \mathrm{~S} 375.0910$, found 375.0912 .

(Z)-3,5-Diphenyl- $N$-(m-tolyl)-1,3,4-thiadiazol-2(3H)-imine $\quad(4 \mathrm{~h}) . \quad 1 \mathrm{~h}$, eluent: EtOAc/PE 1:500; yield: $105 \mathrm{mg}, 61 \%$; white solid, mp 90-92 ${ }^{\circ} \mathrm{C} ;{ }^{1} \mathrm{H}$ NMR (400 MHz, $\left.\mathrm{CDCl}_{3}\right) \delta 8.12-8.09(\mathrm{~m}, 2 \mathrm{H}), 7.71-7.69(\mathrm{~m}, 2 \mathrm{H}), 7.48-7.44(\mathrm{~m}, 2 \mathrm{H}), 7.43-7.40(\mathrm{~m}$, $3 \mathrm{H}), 7.27(\mathrm{t}, J=7.6 \mathrm{~Hz}, 2 \mathrm{H}), 6.96-6.89(\mathrm{~m}, 3 \mathrm{H}), 2.36(\mathrm{~s}, 3 \mathrm{H}) ;{ }^{13} \mathrm{C}$ NMR (100 MHz, $\left.\mathrm{CDCl}_{3}\right) \delta 155.3,152.5,146.6,139.9,139.6,130.6,130.5,129.6,128.9,128.8,126.2$, 126.0, 124.9, 122.2, 121.8, 117.7, 21.6; HRMS (ESI-TOF) $\mathrm{m} / \mathrm{z}:[\mathrm{M}+\mathrm{H}]^{+}$calcd for 
$\mathrm{C}_{21} \mathrm{H}_{18} \mathrm{~N}_{3} \mathrm{~S}$ 344.1216, found 344.1218.

(Z)-3,5-Diphenyl- $N$-(o-tolyl)-1,3,4-thiadiazol-2(3H)-imine (4i). 1 h, eluent: EtOAc/PE 1:500; yield: $88 \mathrm{mg}, 51 \%$; white solid, mp 89-91 ${ }^{\circ} \mathrm{C}$; ${ }^{1} \mathrm{H}$ NMR (400 MHz, $\left.\mathrm{CDCl}_{3}\right) \delta 8.18-8.15(\mathrm{~m}, 2 \mathrm{H}), 7.69-7.67(\mathrm{~m}, 2 \mathrm{H}), 7.48-7.44(\mathrm{~m}, 2 \mathrm{H}), 7.41-7.38(\mathrm{~m}$, $3 \mathrm{H}), 7.28-7.20$ (m, 3H, overlapped with the peak of chloroform), 7.05 (td, $J=7.6,1.2$ $\mathrm{Hz}, 1 \mathrm{H}), 7.00(\mathrm{~d}, J=7.6 \mathrm{~Hz}, 1 \mathrm{H}), 2.26(\mathrm{~s}, 3 \mathrm{H}) ;{ }^{13} \mathrm{C} \mathrm{NMR}\left(100 \mathrm{MHz}, \mathrm{CDCl}_{3}\right) \delta 154.9$, $151.4,146.7,139.9,130.8,130.5,130.4,130.2,128.8,128.7,127.2,126.1,125.9$, 124.1, 122.0, 119.2, 18.0; HRMS (ESI-TOF) m/z: $[\mathrm{M}+\mathrm{H}]^{+}$calcd for $\mathrm{C}_{21} \mathrm{H}_{18} \mathrm{~N}_{3} \mathrm{~S}$ 344.1216, found 344.1218 .

(Z)-N-(2,6-Dichlorophenyl)-3,5-diphenyl-1,3,4-thiadiazol-2(3H)-imine (4j). $4 \mathrm{~h}$, eluent: EtOAc/PE 1:500; yield: 98 mg, 49\%; white solid, mp 177-179 ${ }^{\circ} \mathrm{C}$; ${ }^{1} \mathrm{H}$ NMR $\left(400 \mathrm{MHz}, \mathrm{CDCl}_{3}\right) \delta$ 8.15-8.12 (m, 2H), 7.69-7.66 (m, 2H), 7.52-7.47 (m, 2H), $7.42-7.39(\mathrm{~m}, 3 \mathrm{H}), 7.36(\mathrm{~d}, J=8.0 \mathrm{~Hz}, 2 \mathrm{H}), 7.33-7.29(\mathrm{~m}, 1 \mathrm{H}), 6.98$ (t, $J=8.0 \mathrm{~Hz}$, $1 \mathrm{H}) ;{ }^{13} \mathrm{C} \mathrm{NMR}\left(100 \mathrm{MHz}, \mathrm{CDCl}_{3}\right) \delta 158.1,147.8,146.7,139.2,130.7,130.0,128.91$, 128.87, 128.7, 127.8, 126.6, 126.1, 124.6, 122.8; HRMS (ESI-TOF) m/z: $[\mathrm{M}+\mathrm{H}]^{+}$ calcd for $\mathrm{C}_{20} \mathrm{H}_{14} \mathrm{Cl}_{2} \mathrm{~N}_{3} \mathrm{~S} 398.0280$, found 398.0283 .

(Z)-N-(3,5-Diphenyl-1,3,4-thiadiazol-2(3H)-ylidene)benzamide $(4 \mathrm{k}){ }^{3} 4 \mathrm{~h}$, eluent: EtOAc/PE 1:100; yield: $126 \mathrm{mg}, 71 \%$; white solid, mp $171-173{ }^{\circ} \mathrm{C} ;{ }^{1} \mathrm{H}$ NMR (400 $\left.\mathrm{MHz}, \mathrm{CDCl}_{3}\right) \delta 8.29-8.27(\mathrm{~m}, 2 \mathrm{H}), 8.11-8.08(\mathrm{~m}, 2 \mathrm{H}), 7.93-7.91(\mathrm{~m}, 2 \mathrm{H}), 7.61-7.57$ $(\mathrm{m}, 2 \mathrm{H}), 7.53-7.42(\mathrm{~m}, 7 \mathrm{H}) ;{ }^{13} \mathrm{C}$ NMR $\left(100 \mathrm{MHz}, \mathrm{CDCl}_{3}\right) \delta 174.7,164.8,156.2$, $139.4,135.8,132.3,131.2,129.9,129.7,129.2,128.8,128.2,128.1,126.7,124.6$; HRMS (ESI-TOF) m/z: $[\mathrm{M}+\mathrm{H}]^{+}$calcd for $\mathrm{C}_{21} \mathrm{H}_{16} \mathrm{~N}_{3} \mathrm{OS} 358.1009$, found 358.1011.

(Z)-N,3-Diphenyl-5-(p-tolyl)-1,3,4-thiadiazol-2(3H)-imine $(\mathbf{4 m}){ }^{4}{ }^{4} \mathrm{~h}$, eluent: EtOAc/PE 1:300; yield: $118 \mathrm{mg}, 69 \%$; white solid, mp $118-119{ }^{\circ} \mathrm{C} ;{ }^{1} \mathrm{H}$ NMR (400 $\left.\mathrm{MHz}, \mathrm{CDCl}_{3}\right) \delta 8.12-8.10(\mathrm{~m}, 2 \mathrm{H}), 7.59(\mathrm{~d}, J=8.4 \mathrm{~Hz}, 2 \mathrm{H}), 7.49-7.44(\mathrm{~m}, 2 \mathrm{H})$, 7.40-7.36 (m, 2H), 7.29-7.27 (m, 1H), 7.22 (d, $J=8.0 \mathrm{~Hz}, 2 \mathrm{H}), 7.14-7.08$ (m, 3H), $2.38(\mathrm{~s}, 3 \mathrm{H}) ;{ }^{13} \mathrm{C} \mathrm{NMR}\left(100 \mathrm{MHz}, \mathrm{CDCl}_{3}\right) \delta 155.6,152.6,146.7,140.9,139.9,129.7$, 129.6, 128.8, 127.8, 126.1, 126.0, 124.0, 122.3, 121.0, 21.5; HRMS (ESI-TOF) m/z: $[\mathrm{M}+\mathrm{H}]^{+}$calcd for $\mathrm{C}_{21} \mathrm{H}_{18} \mathrm{~N}_{3} \mathrm{~S} 344.1216$, found 344.1217 .

(Z)-5-(4-Methoxyphenyl)- $N, 3$-diphenyl-1,3,4-thiadiazol-2(3H)-imine (4n). $2 \mathrm{~h}$, 
eluent: EtOAc/PE 1:200; yield: $113 \mathrm{mg}, 63 \%$; white solid, mp $123-125{ }^{\circ} \mathrm{C}$; ${ }^{1} \mathrm{H}$ NMR $\left(400 \mathrm{MHz}, \mathrm{CDCl}_{3}\right) \delta$ 8.12-8.09 (m, 2H), 7.65-7.61 (m, 2H), 7.49-7.44 (m, 2H), 7.40-7.36 (m, 2H), 7.28-7.24 (m, 1H, overlapped with the peak of chloroform), 7.14-7.08 (m, 3H), 6.94-6.90 (m, 2H), $\left.3.84(\mathrm{~s}, 3 \mathrm{H}) ;{ }^{13} \mathrm{C} \mathrm{NMR} \mathrm{(100} \mathrm{MHz,} \mathrm{CDCl}_{3}\right) \delta$ $161.4,155.6,152.6,146.4,139.9,129.7,128.8,127.7,125.9,123.9,123.2,122.2$, 121.0, 114.3, 55.5; HRMS (ESI-TOF) m/z: $[\mathrm{M}+\mathrm{H}]^{+}$calcd for $\mathrm{C}_{21} \mathrm{H}_{18} \mathrm{~N}_{3} \mathrm{OS} 360.1165$, found 360.1167 .

(Z)-5-(4-Chlorophenyl)- $N, 3-d i p h e n y l-1,3,4-t h i a d i a z o l-2(3 H)$-imine $\quad$ (40). $1.5 \mathrm{~h}$, eluent: EtOAc/PE 1:300; yield: $121 \mathrm{mg}, 67 \%$; white solid, mp $117-119{ }^{\circ} \mathrm{C} ;{ }^{1} \mathrm{H}$ NMR $\left(400 \mathrm{MHz}, \mathrm{CDCl}_{3}\right) \delta$ 8.09-8.06 (m, 2H), 7.64-7.61 (m, 2H), 7.49-7.44 (m, 2H), 7.40-7.36 (m, 4H), 7.31-7.26 (m, 1H), 7.15-7.11 (m, 1H), 7.09-7.06 (m, 2H); ${ }^{13} \mathrm{C}$ NMR $\left(100 \mathrm{MHz}, \mathrm{CDCl}_{3}\right) \delta 155.3,152.4,145.4,139.7,136.4,129.7,129.2,129.0$, 128.8, 127.3, 126.3, 124.2, 122.3, 120.9; HRMS (ESI-TOF) m/z: $[\mathrm{M}+\mathrm{H}]^{+}$calcd for $\mathrm{C}_{20} \mathrm{H}_{15} \mathrm{ClN}_{3} \mathrm{~S} 364.0670$, found 364.0671.

(Z)-4-(4-Phenyl-5-(phenylimino)-4,5-dihydro-1,3,4-thiadiazol-2-yl)benzonitrile

(4p). 1 h, eluent: EtOAc/PE 1:60; yield: $115 \mathrm{mg}, 65 \%$; yellow solid, mp 154-156 ${ }^{\circ} \mathrm{C}$; ${ }^{1} \mathrm{H}$ NMR (400 MHz, $\mathrm{CDCl}_{3}$ ) $\delta$ 8.07-8.04 (m, 2H), 7.79-7.76 (m, 2H), 7.70-7.67 (m, 2H), 7.51-7.46 (m, 2H), 7.42-7.37 (m, 2H), 7.33-7.29 (m, 1H), 7.17-7.13 (m, 1H), 7.08-7.05 (m, 2H); ${ }^{13} \mathrm{C}$ NMR (100 MHz, $\left.\mathrm{CDCl}_{3}\right) \delta 154.8,152.1,144.3,139.4,134.5$, $132.7,129.8,128.9,126.7,126.5,124.4,122.5,120.7,118.2,113.6$; HRMS (ESI-TOF) $\mathrm{m} / \mathrm{z}$ : $[\mathrm{M}+\mathrm{H}]^{+}$calcd for $\mathrm{C}_{21} \mathrm{H}_{15} \mathrm{~N}_{4} \mathrm{~S} 355.1012$, found 355.1014 .

(Z)-5-(4-Nitrophenyl)- $N, 3-d i p h e n y l-1,3,4-t h i a d i a z o l-2(3 H)$-imine (4q). ${ }^{5} 2$ h, eluent: EtOAc/PE 1:150; yield: $115 \mathrm{mg}, 61 \%$; red solid, mp 154-156 ${ }^{\circ} \mathrm{C} ;{ }^{1} \mathrm{H}$ NMR (400 MHz, $\left.\mathrm{CDCl}_{3}\right) \delta 8.30-8.26(\mathrm{~m}, 2 \mathrm{H}), 8.08-8.05(\mathrm{~m}, 2 \mathrm{H}), 7.87-7.84(\mathrm{~m}, 2 \mathrm{H}), 7.52-7.47(\mathrm{~m}$, 2H), 7.43-7.38 (m, 2H), $7.33(\mathrm{tt}, J=7.6,1.6 \mathrm{~Hz}, 1 \mathrm{H}), 7.16(\mathrm{tt}, J=7.6,1.2 \mathrm{~Hz}, 1 \mathrm{H})$, 7.10-7.06 (m, 2H); $\left.{ }^{13} \mathrm{C} \mathrm{NMR} \mathrm{(100} \mathrm{MHz,} \mathrm{CDCl}_{3}\right) \delta 154.8,152.0,148.5,143.9,139.3$, 136.2, 129.8, 128.9, 126.8, 126.7, 124.5, 124.3, 122.5, 120.7; HRMS (ESI-TOF) m/z: $[\mathrm{M}+\mathrm{H}]^{+}$calcd for $\mathrm{C}_{20} \mathrm{H}_{15} \mathrm{~N}_{4} \mathrm{O}_{2} \mathrm{~S} 375.0910$, found 375.0912 .

(Z)-5-(3-Chlorophenyl)- $N, 3-d i p h e n y l-1,3,4-t h i a d i a z o l-2(3 H)$-imine (4r). 1 h, eluent: EtOAc/PE 1:800; yield: $138 \mathrm{mg}, 76 \%$; colorless oil; ${ }^{1} \mathrm{H}$ NMR $\left(400 \mathrm{MHz}, \mathrm{CDCl}_{3}\right) \delta$ 
8.10-8.07 (m, 2H), 7.74-7.73 (m, 1H), $7.53(\mathrm{dt}, J=7.6,1.6 \mathrm{~Hz}, 1 \mathrm{H}), 7.50-7.45(\mathrm{~m}$, 2H), 7.41-7.32 (m, 4H), 7.29 (tt, $J=7.6,1.6 \mathrm{~Hz}, 1 \mathrm{H}), 7.16-7.12(\mathrm{~m}, 1 \mathrm{H}), 7.10-7.06$ $(\mathrm{m}, 2 \mathrm{H}) ;{ }^{13} \mathrm{C} \mathrm{NMR}\left(100 \mathrm{MHz}, \mathrm{CDCl}_{3}\right) \delta 155.1,152.3,145.0,139.6,135.1,132.2$, $130.4,130.2,129.8,128.8,126.3,126.0,124.3,124.2,122.3,120.9$; HRMS (ESI-TOF) m/z: [M + H] $]^{+}$calcd for $\mathrm{C}_{20} \mathrm{H}_{15} \mathrm{ClN}_{3} \mathrm{~S}$ 364.0670, found 364.0671.

(Z)-5-(2-Methoxyphenyl)- $N, 3-d i p h e n y l-1,3,4-t h i a d i a z o l-2(3 H)$-imine $(4 \mathrm{~s}) .3 \mathrm{~h}$, eluent: EtOAc/PE 1:150; yield: $83 \mathrm{mg}, 46 \%$; white solid, mp $133-135{ }^{\circ} \mathrm{C} ;{ }^{1} \mathrm{H}$ NMR $\left(400 \mathrm{MHz}, \mathrm{CDCl}_{3}\right) \delta 8.14-8.11(\mathrm{~m}, 2 \mathrm{H}), 8.09(\mathrm{dd}, J=7.6,1.6 \mathrm{~Hz}, 1 \mathrm{H}), 7.48-7.43(\mathrm{~m}$, 2H), 7.41-7.35 (m, 3H), 7.28-7.24 (m, 1H, overlapped with the peak of chloroform), 7.12-7.07 (m, 3H), 7.06-7.02 (m, 1H), 6.95-6.93 (m, 1H), $3.84(\mathrm{~s}, 3 \mathrm{H}) ;{ }^{13} \mathrm{C} \mathrm{NMR}$ $\left(100 \mathrm{MHz}, \mathrm{CDCl}_{3}\right) \delta 157.0,156.5,152.3,142.9,139.9,131.5,129.6,128.7,127.7$, $125.9,123.5,122.3,121.2,121.1,119.4,111.7,55.8 ;$ HRMS (ESI-TOF) m/z: [M + $\mathrm{H}]^{+}$calcd for $\mathrm{C}_{21} \mathrm{H}_{18} \mathrm{~N}_{3} \mathrm{OS} 360.1165$, found 360.1168 .

(Z)-5-(2-Fluorophenyl)- $N, 3-d i p h e n y l-1,3,4-t h i a d i a z o l-2(3 H)$-imine (4t). 2 h, eluent: EtOAc/PE 1:300; yield: $122 \mathrm{mg}, 70 \%$; white solid, mp 153-155 ${ }^{\circ} \mathrm{C} ;{ }^{1} \mathrm{H}$ NMR (400 $\left.\mathrm{MHz} \mathrm{CDCl}_{3}\right) \delta 8.12-8.09(\mathrm{~m}, 2 \mathrm{H}), 7.99(\mathrm{td}, J=7.6,1.6 \mathrm{~Hz}, 1 \mathrm{H}), 7.50-7.45(\mathrm{~m}, 2 \mathrm{H})$, 7.43-7.36 (m, 3H), 7.31-7.27 (m, 1H), 7.25-7.20 (m, 1H, overlapped with the peak of chloroform), 7.16-7.08 (m, 4H); ${ }^{13} \mathrm{C}$ NMR (100 MHz, $\left.\mathrm{CDCl}_{3}\right) \delta 159.8\left(\mathrm{~d}, J_{\mathrm{C}-\mathrm{F}}=\right.$ $251.7 \mathrm{~Hz}), 155.9\left(\mathrm{~d}, J_{\mathrm{C}-\mathrm{F}}=5.2 \mathrm{~Hz}\right), 152.1,141.0\left(\mathrm{~d}, J_{\mathrm{C}-\mathrm{F}}=4.4 \mathrm{~Hz}\right), 139.7,131.9(\mathrm{~d}$, $\left.J_{\mathrm{C}-\mathrm{F}}=8.4 \mathrm{~Hz}\right), 129.7,128.8,128.1\left(\mathrm{~d}, J_{\mathrm{C}-\mathrm{F}}=2.4 \mathrm{~Hz}\right), 126.2,124.6\left(\mathrm{~d}, J_{\mathrm{C}-\mathrm{F}}=3.4 \mathrm{~Hz}\right)$, 124.0, 122.4, 121.0, $118.6\left(\mathrm{~d}, J_{\mathrm{C}-\mathrm{F}}=10.3 \mathrm{~Hz}\right), 116.5\left(\mathrm{~d}, J_{\mathrm{C}-\mathrm{F}}=21.4 \mathrm{~Hz}\right)$; HRMS (ESI-TOF) m/z: $[\mathrm{M}+\mathrm{H}]^{+}$calcd for $\mathrm{C}_{20} \mathrm{H}_{15} \mathrm{FN}_{3} \mathrm{~S}$ 348.0965, found 348.0966.

(Z)-5-(Naphthalen-2-yl)-N,3-diphenyl-1,3,4-thiadiazol-2(3H)-imine $\quad(4 \mathrm{u}) . \quad 1 \mathrm{~h}$, eluent: EtOAc/PE 1:200; yield: $136 \mathrm{mg}, 72 \%$; white solid, mp $145-146{ }^{\circ} \mathrm{C}$; ${ }^{1} \mathrm{H}$ NMR $\left(400 \mathrm{MHz} \mathrm{CDCl}_{3}\right) \delta$ 8.16-8.13 (m, 2H), 7.98-7.94 (m, 2H), 7.86-7.80 (m, 3H), 7.52-7.46 (m, 4H), 7.42-7.38 (m, 2H), 7.31-7.27 (m, 1H), 7.17-7.10 (m, 3H); ${ }^{13} \mathrm{C}$ NMR $\left(100 \mathrm{MHz}, \mathrm{CDCl}_{3}\right) \delta 155.5,152.6,146.6,139.8,134.2,133.0,129.8,128.84$, 128.76, 128.4, 128.0, 127.9, 127.4, 127.0, 126.6, 126.1, 124.1, 122.6, 122.3, 121.0; HRMS (ESI-TOF) m/z: [M + H] $]^{+}$calcd for $\mathrm{C}_{24} \mathrm{H}_{18} \mathrm{~N}_{3} \mathrm{~S} 380.1216$, found 380.1217 .

(Z)-5-(Furan-2-yl)-N,3-diphenyl-1,3,4-thiadiazol-2(3H)-imine (4v). 2 h, eluent: 
EtOAc/PE 1:200; yield: $74 \mathrm{mg}, 46 \%$; white solid, mp $108-110{ }^{\circ} \mathrm{C} ;{ }^{1} \mathrm{H}$ NMR (400 $\left.\mathrm{MHz}, \mathrm{CDCl}_{3}\right) \delta 8.06-8.03(\mathrm{~m}, 2 \mathrm{H}), 7.50(\mathrm{dd}, J=2.0,0.8 \mathrm{~Hz}, 1 \mathrm{H}), 7.48-7.44(\mathrm{~m}, 2 \mathrm{H})$, 7.39-7.35 (m, 2H), 7.30-7.25 (m, 1H), 7.14-7.10 (m, 1H), 7.08-7.06 (m, 2H), 6.80 $(\mathrm{dd}, J=3.6,0.4 \mathrm{~Hz}, 1 \mathrm{H}), 6.51(\mathrm{q}, J=2.0 \mathrm{~Hz}, 1 \mathrm{H}) ;{ }^{13} \mathrm{C} \mathrm{NMR}\left(100 \mathrm{MHz}, \mathrm{CDCl}_{3}\right) \delta$ $154.8,152.3,145.1,144.3,139.5,137.9,129.7,128.8,126.2,124.1,122.4,120.8$, 112.1, 110.4; HRMS (ESI-TOF) m/z: $[\mathrm{M}+\mathrm{H}]^{+}$calcd for $\mathrm{C}_{18} \mathrm{H}_{14} \mathrm{~N}_{3} \mathrm{OS}$ 320.0852, found 320.0854 .

(Z)-5-(tert-Butyl)- $N, 3-d i p h e n y l-1,3,4-t h i a d i a z o l-2(3 H)$-imine $(4 \mathrm{w}) .6 \mathrm{~h}$, eluent: EtOAc/PE 1:300; yield: $86 \mathrm{mg}, 56 \%$; white solid, mp 94-96 ${ }^{\circ} \mathrm{C} ;{ }^{1} \mathrm{H}$ NMR (400 MHz, $\left.\mathrm{CDCl}_{3}\right) \delta 8.06-8.04(\mathrm{~m}, 2 \mathrm{H}), 7.45-7.41(\mathrm{~m}, 2 \mathrm{H}), 7.38-7.34(\mathrm{~m}, 2 \mathrm{H}), 7.24-7.20(\mathrm{~m}$, 1H), 7.11-7.08 (m, 1H), 7.06-7.03 (m, 2H), $1.33(\mathrm{~s}, 9 \mathrm{H}) ;{ }^{13} \mathrm{C}$ NMR (100 MHz, $\left.\mathrm{CDCl}_{3}\right) \delta 158.4,156.5,153.0,140.1,129.7,128.8,125.6,123.8,122.0,121.2,36.8$, 29.6; HRMS (ESI-TOF) m/z: $[\mathrm{M}+\mathrm{H}]^{+}$calcd for $\mathrm{C}_{18} \mathrm{H}_{20} \mathrm{~N}_{3} \mathrm{~S}$ 310.1372, found 310.1373 .

(Z)-5-Cyclohexyl- $N, 3-d i p h e n y l-1,3,4-t h i a d i a z o l-2(3 H)$-imine (4x). 12 h, eluent: EtOAc/PE 1:300; yield: $96 \mathrm{mg}, 57 \%$; white solid, mp 55-57 ${ }^{\circ} \mathrm{C} ;{ }^{1} \mathrm{H}$ NMR (400 MHz, $\left.\mathrm{CDCl}_{3}\right) \delta 8.04-8.01(\mathrm{~m}, 2 \mathrm{H}), 7.45-7.40(\mathrm{~m}, 2 \mathrm{H}), 7.37-7.32(\mathrm{~m}, 2 \mathrm{H}), 7.24-7.20(\mathrm{~m}$, 1H), 7.08 (tt, $J=7.6,1.2 \mathrm{~Hz}, 1 \mathrm{H}), 7.05-7.02(\mathrm{~m}, 2 \mathrm{H}), 2.72(\mathrm{tt}, J=11.2,3.6 \mathrm{~Hz}, 1 \mathrm{H})$, $2.02-1.98(\mathrm{~m}, 2 \mathrm{H}), 1.83-1.79(\mathrm{~m}, 2 \mathrm{H}), 1.72-1.67(\mathrm{~m}, 1 \mathrm{H}), 1.49-1.17(\mathrm{~m}, 5 \mathrm{H}) ;{ }^{13} \mathrm{C}$ NMR $\left(100 \mathrm{MHz}, \mathrm{CDCl}_{3}\right) \delta 155.9,154.5,152.8,139.9,129.5,128.7,125.6,123.7$, 121.9, 121.0, 40.8, 32.0, 25.63, 25.61; HRMS (ESI-TOF) $\mathrm{m} / \mathrm{z}:[\mathrm{M}+\mathrm{H}]^{+}$calcd for $\mathrm{C}_{20} \mathrm{H}_{22} \mathrm{~N}_{3} \mathrm{~S} 336.1529$, found 336.1531 .

(Z)-5-Isopropyl- $N, 3-d i p h e n y l-1,3,4-t h i a d i a z o l-2(3 H)$-imine $\mathbf{( 4 y )} 3 \mathrm{~h}$, eluent: EtOAc/PE 1:400; yield: $89 \mathrm{mg}, 60 \%$; white solid, mp 58-60 ${ }^{\circ} \mathrm{C} ;{ }^{1} \mathrm{H}$ NMR (400 MHz, $\left.\mathrm{CDCl}_{3}\right) \delta 8.04-8.01(\mathrm{~m}, 2 \mathrm{H}), 7.45-7.40(\mathrm{~m}, 2 \mathrm{H}), 7.37-7.33(\mathrm{~m}, 2 \mathrm{H}), 7.24-7.20(\mathrm{~m}$, $1 \mathrm{H}$, overlapped with the peak of chloroform), 7.11-7.07 (m, 1H), 7.05-7.03 (m, 2H), 3.02 (hept, $J=7.2 \mathrm{~Hz}, 1 \mathrm{H}), 1.29(\mathrm{~d}, J=6.8 \mathrm{~Hz}, 6 \mathrm{H}) ;{ }^{13} \mathrm{C} \mathrm{NMR}\left(100 \mathrm{MHz}, \mathrm{CDCl}_{3}\right) \delta$ $156.0,155.4,152.8,139.9,129.6,128.7,125.7,123.8,122.0,121.0,31.9,21.6$; HRMS (ESI-TOF) m/z: [M + H] $]^{+}$calcd for $\mathrm{C}_{17} \mathrm{H}_{18} \mathrm{~N}_{3} \mathrm{~S} 296.1216$, found 296.1219.

(Z)-5-Ethyl- $N, 3$-diphenyl-1,3,4-thiadiazol-2(3H)-imine (4z). 7 h, eluent: EtOAc/PE 
1:400; yield: $95 \mathrm{mg}, 67 \%$; white solid, mp 42-44 ${ }^{\circ} \mathrm{C} ;{ }^{1} \mathrm{H}$ NMR $\left(400 \mathrm{MHz}, \mathrm{CDCl}_{3}\right) \delta$ 8.03-8.00 (m, 2H), 7.45-7.40 (m, 2H), 7.37-7.32 (m, 2H), 7.25-7.20 (m, 1H, overlapped with the peak of chloroform), 7.11-7.07 (m, 1H), 7.04-7.02 (m, 2H), 2.72 $(\mathrm{q}, J=7.6 \mathrm{~Hz}, 2 \mathrm{H}), 1.27(\mathrm{t}, J=7.6 \mathrm{~Hz}, 3 \mathrm{H}) ;{ }^{13} \mathrm{C} \mathrm{NMR}\left(100 \mathrm{MHz}, \mathrm{CDCl}_{3}\right) \delta 156.1$, $152.7,150.9,139.8,129.6,128.8,125.7,123.8,122.0,121.0,25.3,12.5$; HRMS (ESI-TOF) m/z: $[\mathrm{M}+\mathrm{H}]^{+}$calcd for $\mathrm{C}_{16} \mathrm{H}_{16} \mathrm{~N}_{3} \mathrm{~S} 282.1059$, found 282.1061.

(Z)-N,5-Diphenyl-3-(p-tolyl)-1,3,4-thiadiazol-2(3H)-imine $(\mathbf{4 a a}){ }^{4} 3 \mathrm{~h}$, eluent: EtOAc/PE 1:250; yield: $89 \mathrm{mg}, 52 \%$; white solid, mp 94-96 ${ }^{\circ} \mathrm{C} ;{ }^{1} \mathrm{H}$ NMR (400 MHz, $\left.\mathrm{CDCl}_{3}\right) \delta 7.93(\mathrm{~d}, J=8.4 \mathrm{~Hz}, 2 \mathrm{H}), 7.70-7.68(\mathrm{~m}, 2 \mathrm{H}), 7.43-7.36(\mathrm{~m}, 5 \mathrm{H}), 7.27(\mathrm{~d}, J=$ $8.4 \mathrm{~Hz}, 2 \mathrm{H}), 7.14-7.07$ (m, 3H), 2.39 (s, 3H); ${ }^{13} \mathrm{C} \mathrm{NMR}\left(100 \mathrm{MHz}, \mathrm{CDCl}_{3}\right) \delta$ 155.6, $152.6,146.3,137.3,136.1,130.6,130.4,129.7,129.4,128.9,126.1,123.9,122.5$, 121.0, 21.1; HRMS (ESI-TOF) $\mathrm{m} / \mathrm{z}$ : $[\mathrm{M}+\mathrm{H}]^{+}$calcd for $\mathrm{C}_{21} \mathrm{H}_{18} \mathrm{~N}_{3} \mathrm{~S} 344.1216$, found 344.1217.

(Z)-3-(4-Chlorophenyl)- $N, 5$-diphenyl-1,3,4-thiadiazol-2(3H)-imine $\quad$ (4ab). $1 \mathrm{~h}$, eluent: EtOAc/PE 1:500; yield: $145 \mathrm{mg}, 80 \%$; white solid, mp $142-143{ }^{\circ} \mathrm{C} ;{ }^{1} \mathrm{H}$ NMR $\left(400 \mathrm{MHz}, \mathrm{CDCl}_{3}\right) \delta 8.13-8.11(\mathrm{~m}, 2 \mathrm{H}), 7.71-7.68(\mathrm{~m}, 2 \mathrm{H}), 7.44-7.38(\mathrm{~m}, 7 \mathrm{H}), 7.15$ $(\operatorname{td}, J=7.2,0.8 \mathrm{~Hz}, 1 \mathrm{H}), 7.09-7.07(\mathrm{~m}, 2 \mathrm{H}) ;{ }^{13} \mathrm{C} \mathrm{NMR}\left(100 \mathrm{MHz}, \mathrm{CDCl}_{3}\right) \delta 155.2$, $152.2,147.0,138.4,131.1,130.7,130.3,129.8,129.0,128.8,126.2,124.2,123.2$, 120.9; HRMS (ESI-TOF) m/z: $[\mathrm{M}+\mathrm{H}]^{+}$calcd for $\mathrm{C}_{20} \mathrm{H}_{15} \mathrm{ClN}_{3} \mathrm{~S} 364.0670$, found 364.0673.

(Z)-4-(5-Phenyl-2-(phenylimino)-1,3,4-thiadiazol-3(2H)-yl)benzonitrile (4ac). $2 \mathrm{~h}$, eluent: EtOAc/PE 1:50; yield: $110 \mathrm{mg}, 62 \%$; white solid, mp $159-162{ }^{\circ} \mathrm{C}$; ${ }^{1} \mathrm{H}$ NMR $\left(400 \mathrm{MHz}, \mathrm{CDCl}_{3}\right) \delta 8.45(\mathrm{dt}, J=9.2,2.0 \mathrm{~Hz}, 2 \mathrm{H}), 7.75-7.70(\mathrm{~m}, 4 \mathrm{H}), 7.48-7.40(\mathrm{~m}$, 5H), 7.20-7.16 (m, 1H), 7.10-7.07 (m, 2H); $\left.{ }^{13} \mathrm{C} \mathrm{NMR} \mathrm{(100} \mathrm{MHz,} \mathrm{CDCl}_{3}\right) \delta 154.9$, $151.7,148.2,143.5,132.9,131.1,129.91,129.87,129.1,126.4,124.7,121.1,120.7$, 118.9, 108.2; HRMS (ESI-TOF) m/z: $[\mathrm{M}+\mathrm{H}]^{+}$calcd for $\mathrm{C}_{21} \mathrm{H}_{15} \mathrm{~N}_{4} \mathrm{~S} 355.1012$, found 355.1015 .

(Z)-3-(4-Nitrophenyl)- $N, 5$-diphenyl-1,3,4-thiadiazol-2(3H)-imine $\quad(4 \mathrm{ad}) .^{6} \quad 1 \mathrm{~h}$, eluent: $\mathrm{CH}_{2} \mathrm{Cl}_{2} / \mathrm{PE} \mathrm{1:8;} \mathrm{yield:} 130 \mathrm{mg}, 70 \%$; yellow solid, mp 204-205 ${ }^{\circ} \mathrm{C}$; ${ }^{1} \mathrm{H}$ NMR $\left(400 \mathrm{MHz}, \mathrm{CDCl}_{3}\right) \delta 8.52(\mathrm{~d}, J=9.2 \mathrm{~Hz}, 2 \mathrm{H}), 8.32(\mathrm{~d}, J=9.2 \mathrm{~Hz}, 2 \mathrm{H}), 7.74-7.72(\mathrm{~m}$, 
2H), 7.49-7.41 (m, 5H), $7.19(\mathrm{t}, J=7.6 \mathrm{~Hz}, 1 \mathrm{H}), 7.10(\mathrm{~d}, J=7.6 \mathrm{~Hz}, 2 \mathrm{H}) ;{ }^{13} \mathrm{C} \mathrm{NMR}$ $\left(100 \mathrm{MHz}, \mathrm{CDCl}_{3}\right) \delta 154.8,151.6,148.6,145.1,144.2,131.3,129.9,129.8,129.1$, 126.4, 124.8, 124.6, 120.7, 120.6; HRMS (ESI-TOF) $\mathrm{m} / \mathrm{z}:[\mathrm{M}+\mathrm{H}]^{+}$calcd for $\mathrm{C}_{20} \mathrm{H}_{15} \mathrm{~N}_{4} \mathrm{O}_{2} \mathrm{~S} 375.0910$, found 375.0907.

(Z)-3-(3-Chlorophenyl)-N,5-diphenyl-1,3,4-thiadiazol-2(3H)-imine (4ae). 1 h, eluent: EtOAc/PE 1:200; yield: 109 mg, 60\%; white solid, mp 141-142 ${ }^{\circ} \mathrm{C} ;{ }^{1} \mathrm{H}$ NMR $\left(400 \mathrm{MHz}, \mathrm{CDCl}_{3}\right) \delta 8.22-8.21(\mathrm{~m}, 1 \mathrm{H}), 8.16-8.13(\mathrm{~m}, 1 \mathrm{H}), 7.72-7.69(\mathrm{~m}, 2 \mathrm{H})$, 7.45-7.36 (m, 6H), 7.25-7.22 (m, 1H, overlapped with the peak of chloroform), 7.17-7.13 (m, 1H), 7.10-7.08 (m, 2H); ${ }^{13} \mathrm{C}$ NMR (100 MHz, $\left.\mathrm{CDCl}_{3}\right) \delta$ 155.0, 152.1, $147.1,140.8,134.3,130.7,130.2,129.72,129.68,128.9,126.2,125.7,124.2,121.7$, 120.8, 119.7; HRMS (ESI-TOF) m/z: $[\mathrm{M}+\mathrm{H}]^{+}$calcd for $\mathrm{C}_{20} \mathrm{H}_{15} \mathrm{ClN}_{3} \mathrm{~S} 364.0670$, found 364.0671 .

(Z)-3-(2-Chlorophenyl)- $N, 5$-diphenyl-1,3,4-thiadiazol-2(3H)-imine (4af). $0.5 \mathrm{~h}$, eluent: EtOAc/PE 1:80; yield: $141 \mathrm{mg}, 78 \%$; colorless oil; ${ }^{1} \mathrm{H}$ NMR (400 MHz, $\mathrm{CDCl}_{3}$ ) א 7.68-7.65 (m, 3H), 7.58-7.56 (m, 1H), 7.45-7.32 (m, 7H), 7.11-7.04 (m, 3H); ${ }^{13} \mathrm{C}$ NMR (100 MHz, $\left.\mathrm{CDCl}_{3}\right) \delta 156.1,152.4,147.1,136.7,132.8,130.9,130.58,130.56$, 130.4, 130.2, 129.6, 129.0, 127.9, 126.2, 124.1, 121.1; HRMS (ESI-TOF) m/z: [M + $\mathrm{H}]^{+}$calcd for $\mathrm{C}_{20} \mathrm{H}_{15} \mathrm{ClN}_{3} \mathrm{~S} 364.0670$, found 364.0672 .

(Z)-3-(tert-Butyl)-N,5-diphenyl-1,3,4-thiadiazol-2(3H)-imine (4ag). $22 \mathrm{~h}$, eluent: EtOAc/PE 1:250; yield: $71 \mathrm{mg}, 46 \%$; colorless oil; ${ }^{1} \mathrm{H}$ NMR (400 $\left.\mathrm{MHz}, \mathrm{CDCl}_{3}\right) \delta$ 7.59-7.57 (m, 2H), 7.38-7.34 (m, 5H), 7.09-7.03 (m, 3H), $1.76(\mathrm{~s}, 9 \mathrm{H}) ;{ }^{13} \mathrm{C} \mathrm{NMR}$ $\left(100 \mathrm{MHz} \mathrm{CDCl}_{3}\right) \delta 155.8,153.0,142.0,131.4,129.6,129.5,128.8,125.7,123.3$, 121.0, 62.1, 28.1; HRMS (ESI-TOF) m/z: $[\mathrm{M}+\mathrm{H}]^{+}$calcd for $\mathrm{C}_{18} \mathrm{H}_{20} \mathrm{~N}_{3} \mathrm{~S} 310.1372$, found 310.1374 . 


\section{References}

1. Wolkoff, P.; Nemeth, S. T.; Gibson, M. S. Reaction of Hydrazonyl Halides with Derivatives of Thiourea and Thiosemicarbazide; A New Source of $C$-Amino- and C-Hydrazino-1,2,4-triazoles. Can. J. Chem. 1975, 53, 3211-3215.

2. Barnikow, G.; Abraham, W. Isothiocyanates. XXVI. Conversion of Amidrazone with Isothiocyanates. Zeitschrift für Chemie 1969, 9, 183-184.

3. Yamamoto, I.; Mamba, A.; Gotoh, H. Heterocyclic Compounds. Part V. Reactions of Phenyl and Benzoyl Isocyanates and Benzoyl Isothiocyanate with Hydrazones. $J$. Chem. Soc., Perkin Trans. 1 1976, 2243-2248.

4. Bose, P. K.; Chaudhury, D. C. R. Thiodiazines. V. J. Indian Chem. Soc. 1927, 4, 257-264.

5. Noto, R.; Lo Meo, P.; Gruttadauria, M.; Werber, G. A Quantitative Study of Substituent Effects on Oxidative Cyclization of Some 2-Aryl-substituted Aldehyde Thiosemicarbazones Induced by Ferric Chloride and Cupric Perchlorate. $J$. Heterocyclic Chem. 1999, 36, 667-674.

6. Butler, R. N.; Ni Bhradaigh, E. P.; Fitzgerald, K. J. Comparative Reactions of Benzonitrilium N-4-Nitrophenylimide with Some 1-Substituted Tetrazol-5-ones, Tetrazole-5-thiones and Tetrazol-5-amines: A Route to 1,2,4-Triazole and 1,3,4-Thiadiazole Derivatives. J. Chem. Res. (S) 1993, 306-307. 
${ }^{1} \mathrm{H}$ NMR $\left(400 \mathrm{MHz}, \mathrm{CDCl}_{3}\right)$

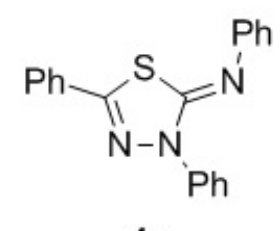

4a

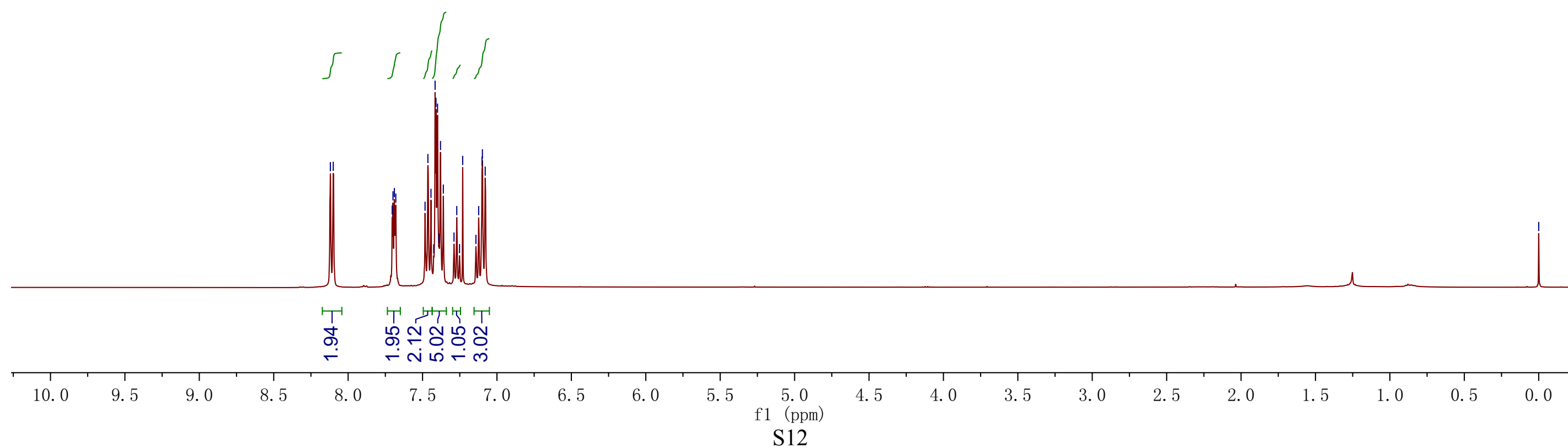


${ }^{13} \mathrm{C} \mathrm{NMR}\left(100 \mathrm{MHz}, \mathrm{CDCl}_{3}\right)$
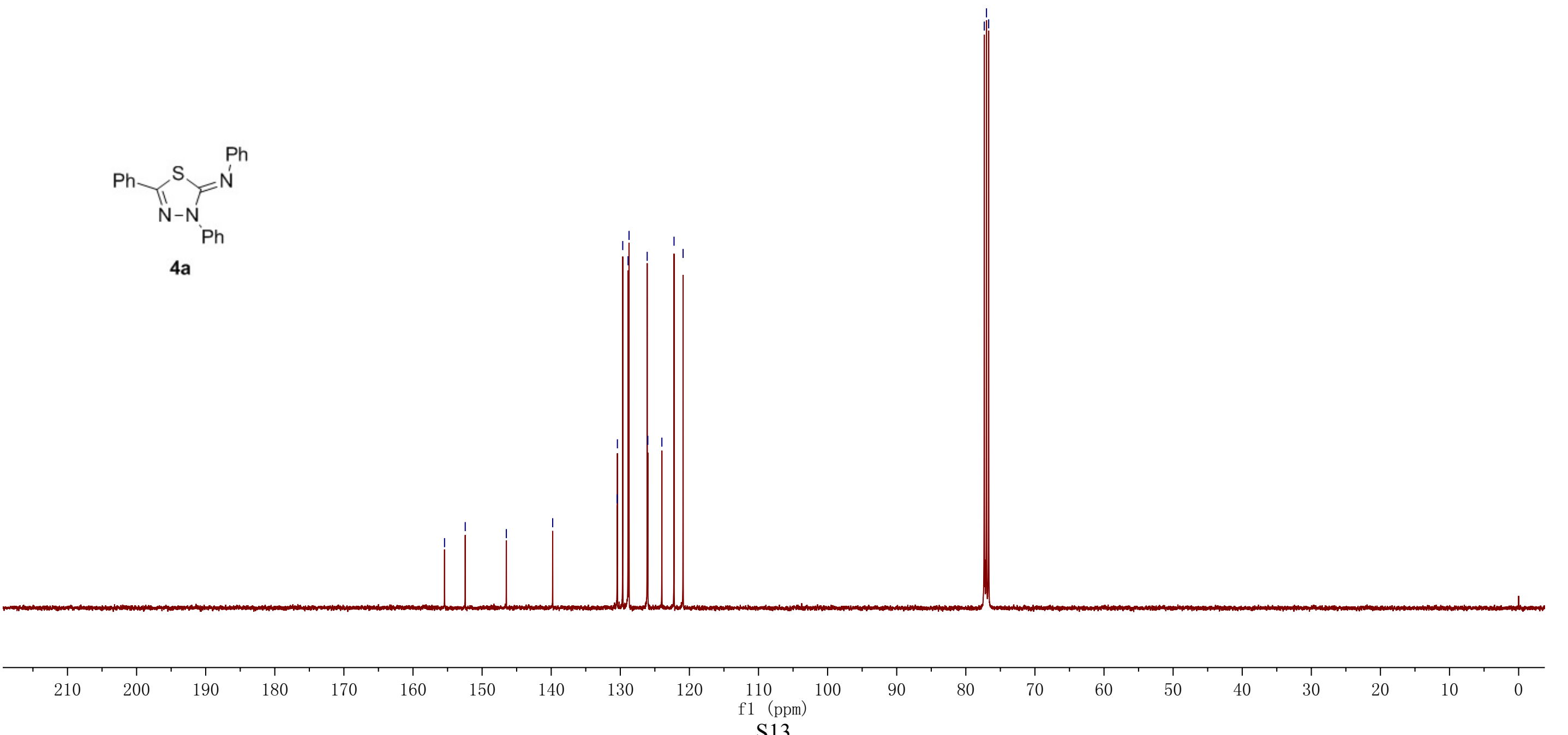
${ }^{1} \mathrm{H}$ NMR (400 MHz, $\left.\mathrm{CDCl}_{3}\right)$

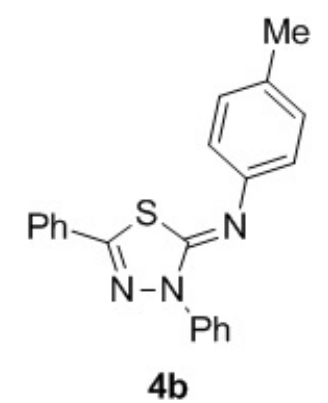

4b

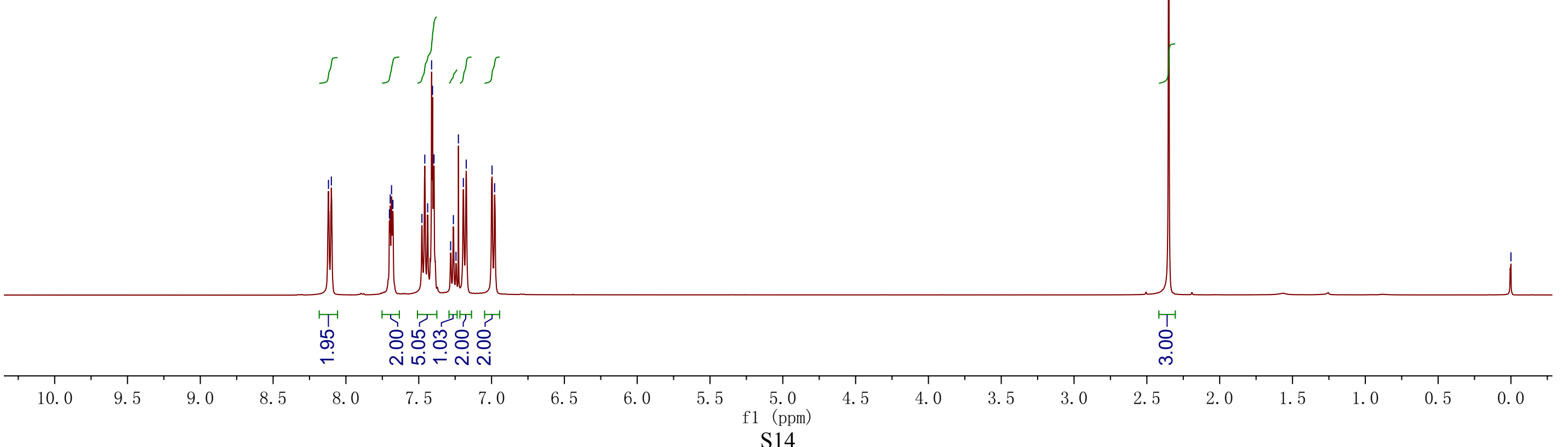


${ }^{13} \mathrm{C}$ NMR $\left(100 \mathrm{MHz}, \mathrm{CDCl}_{3}\right)$

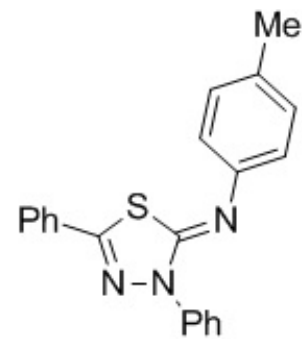

4b

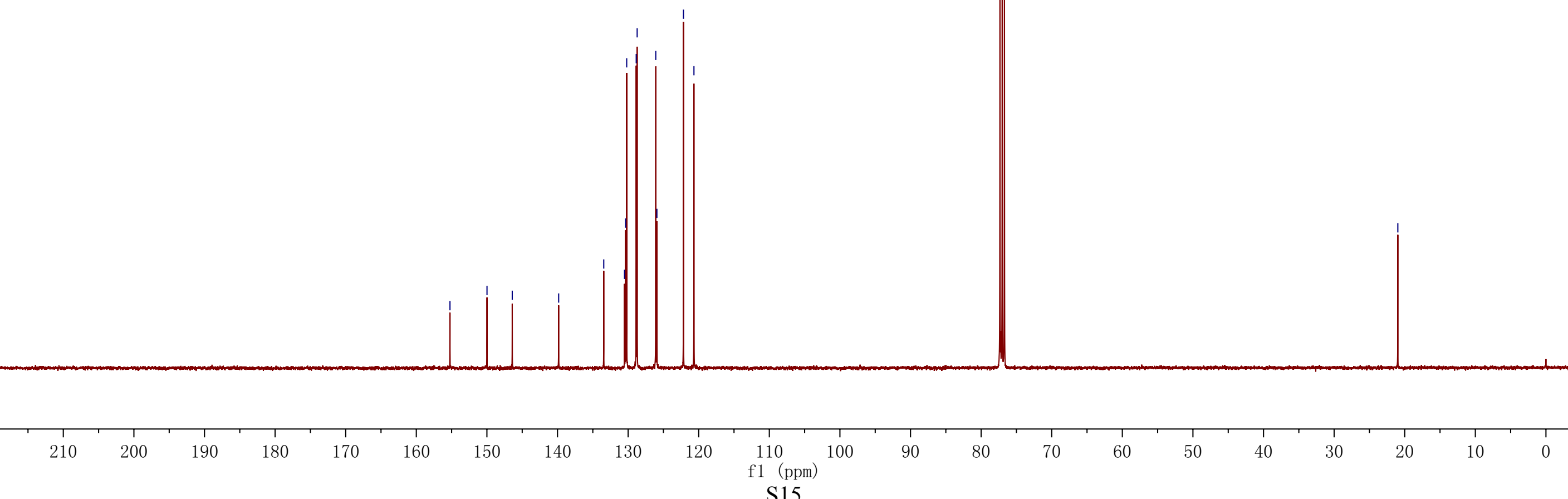


${ }^{1} \mathrm{H}$ NMR $\left(400 \mathrm{MHz}, \mathrm{CDCl}_{3}\right)$

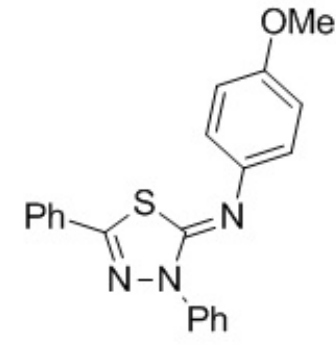

$4 c$
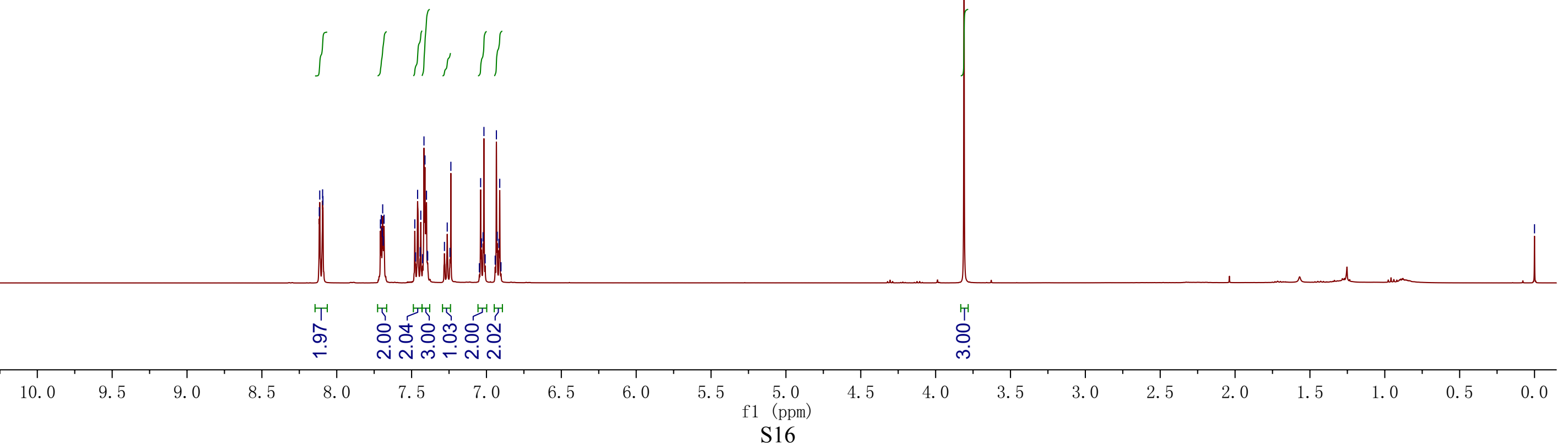
${ }^{13} \mathrm{C}$ NMR (100 MHz, $\mathrm{CDCl}_{3}$ )

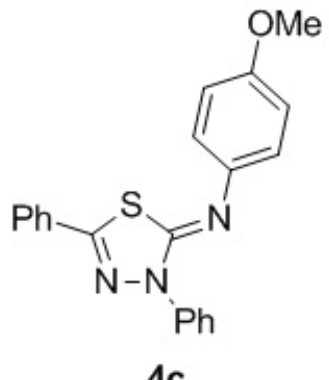

$4 c$

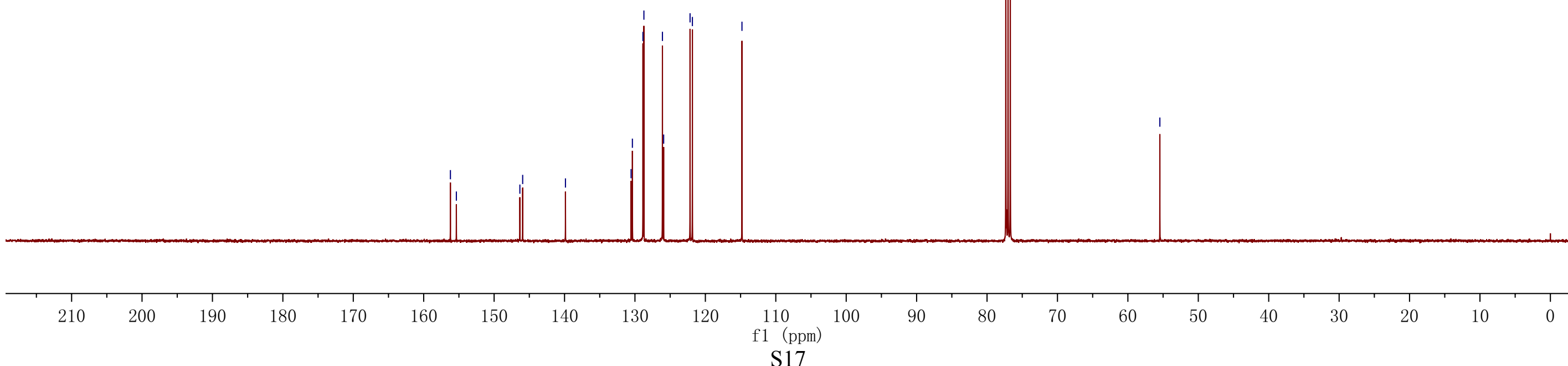



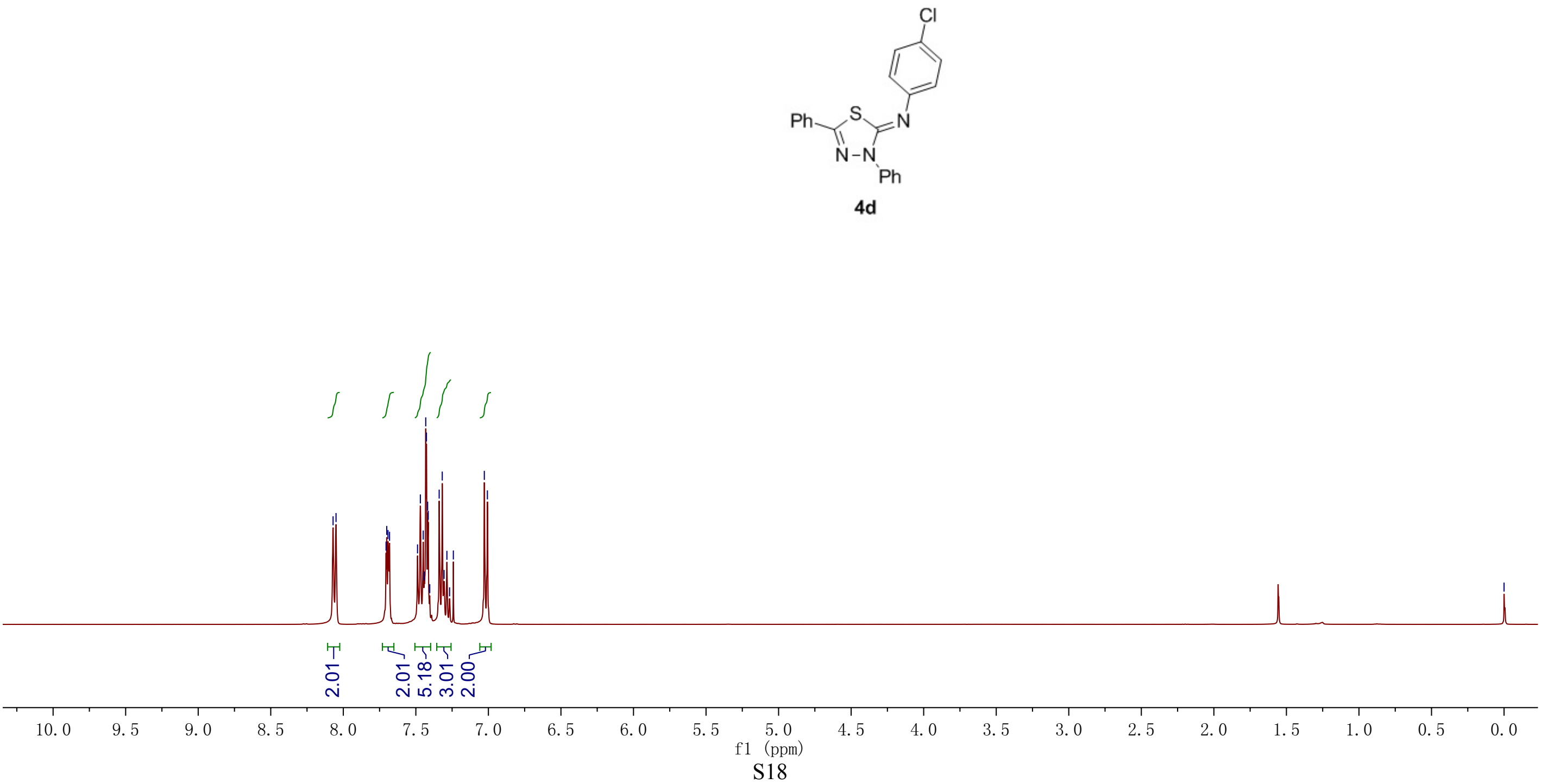
${ }^{13} \mathrm{C}$ NMR (100 MHz, $\left.\mathrm{CDCl}_{3}\right)$
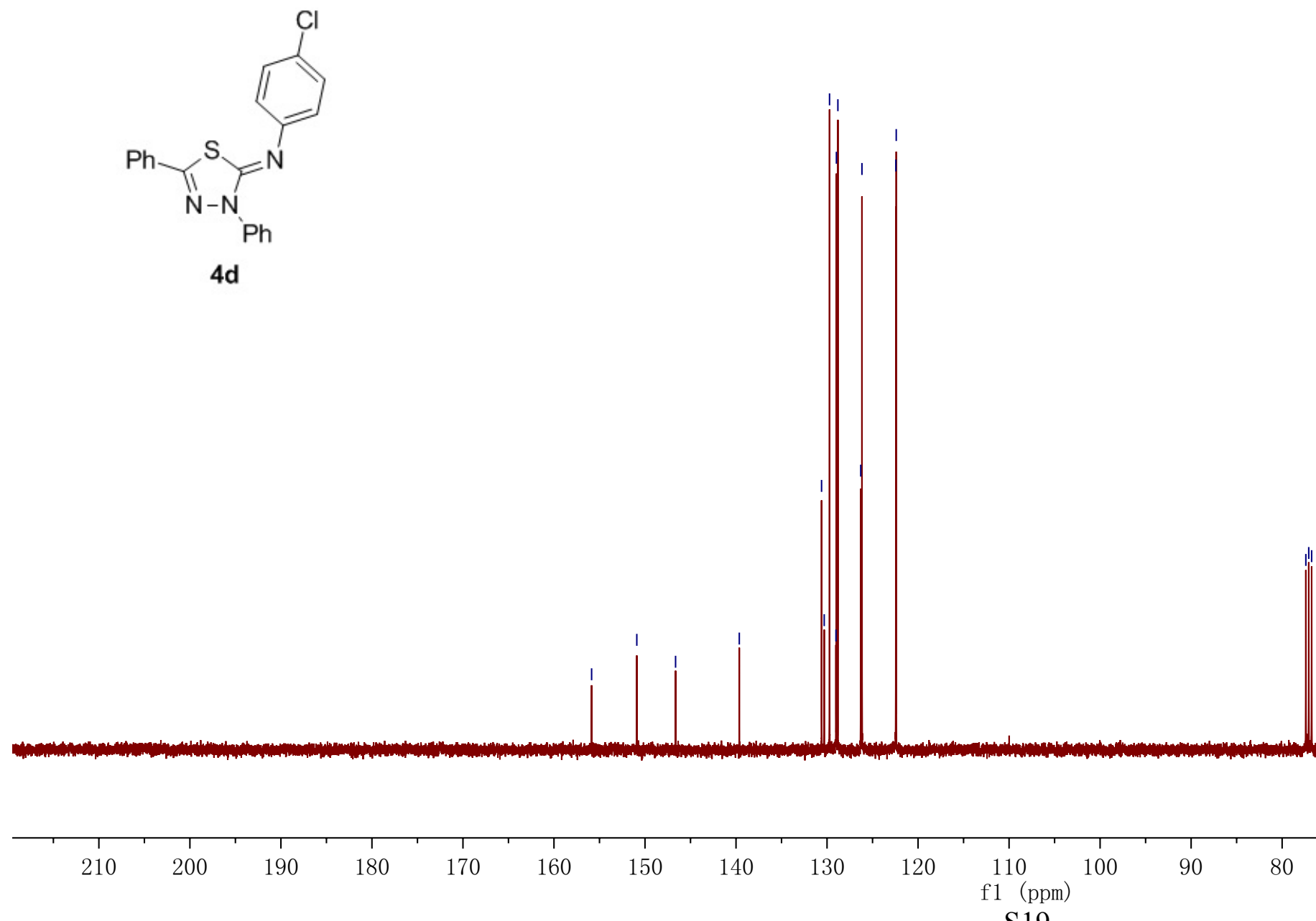
${ }^{1} \mathrm{H}$ NMR $\left(400 \mathrm{MHz}, \mathrm{CDCl}_{3}\right)$
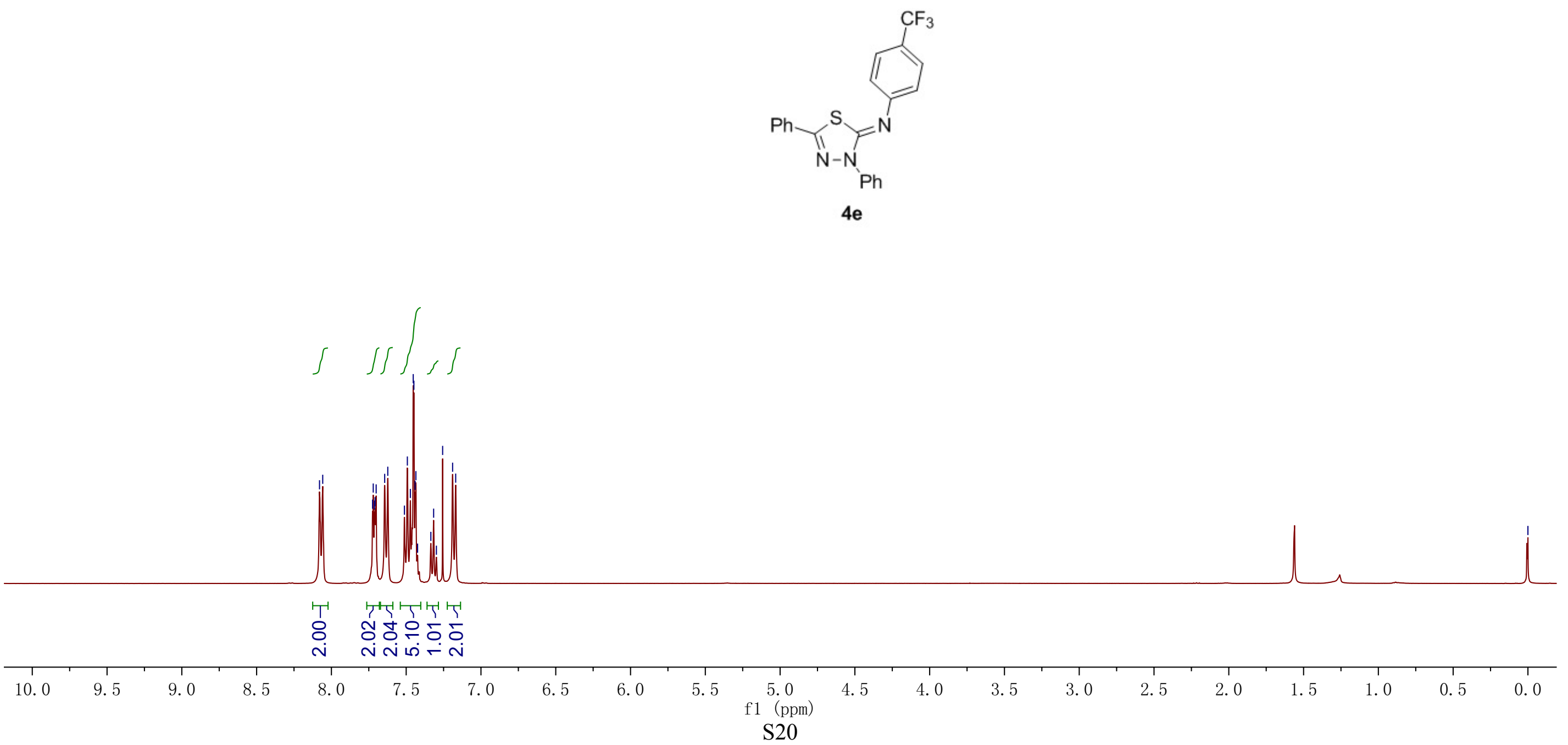
${ }^{13} \mathrm{C}$ NMR $\left(100 \mathrm{MHz}, \mathrm{CDCl}_{3}\right)$

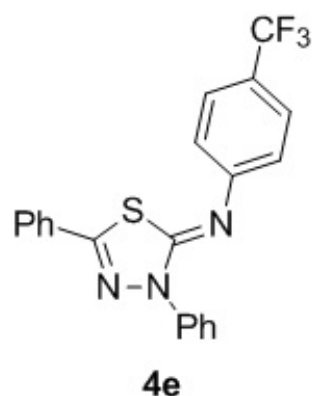

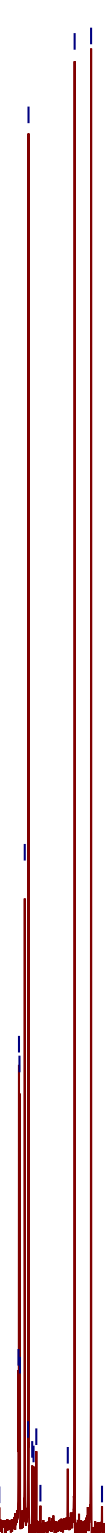

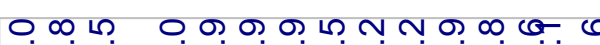

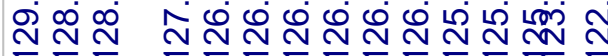

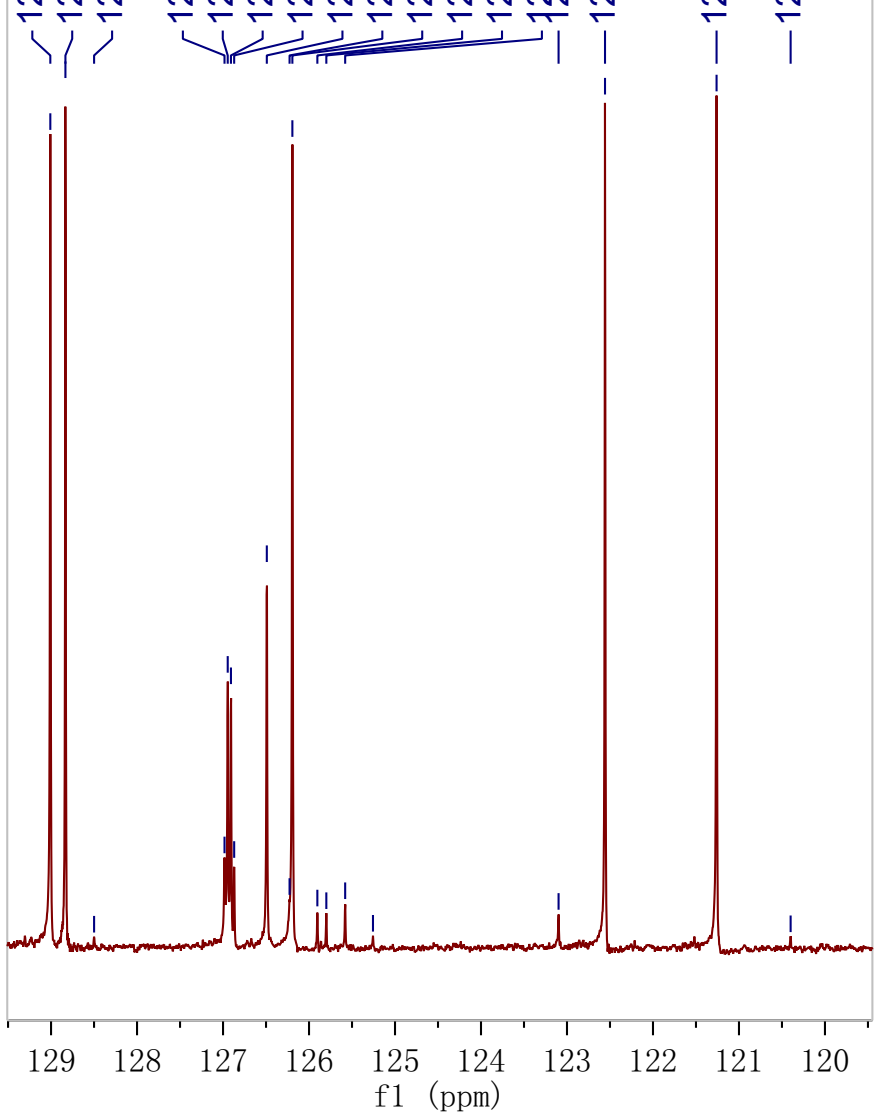

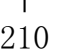

200

190

180

170

160

150

140

130

120

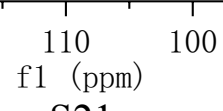


${ }^{1} \mathrm{H}$ NMR $\left(400 \mathrm{MHz}, \mathrm{CDCl}_{3}\right)$
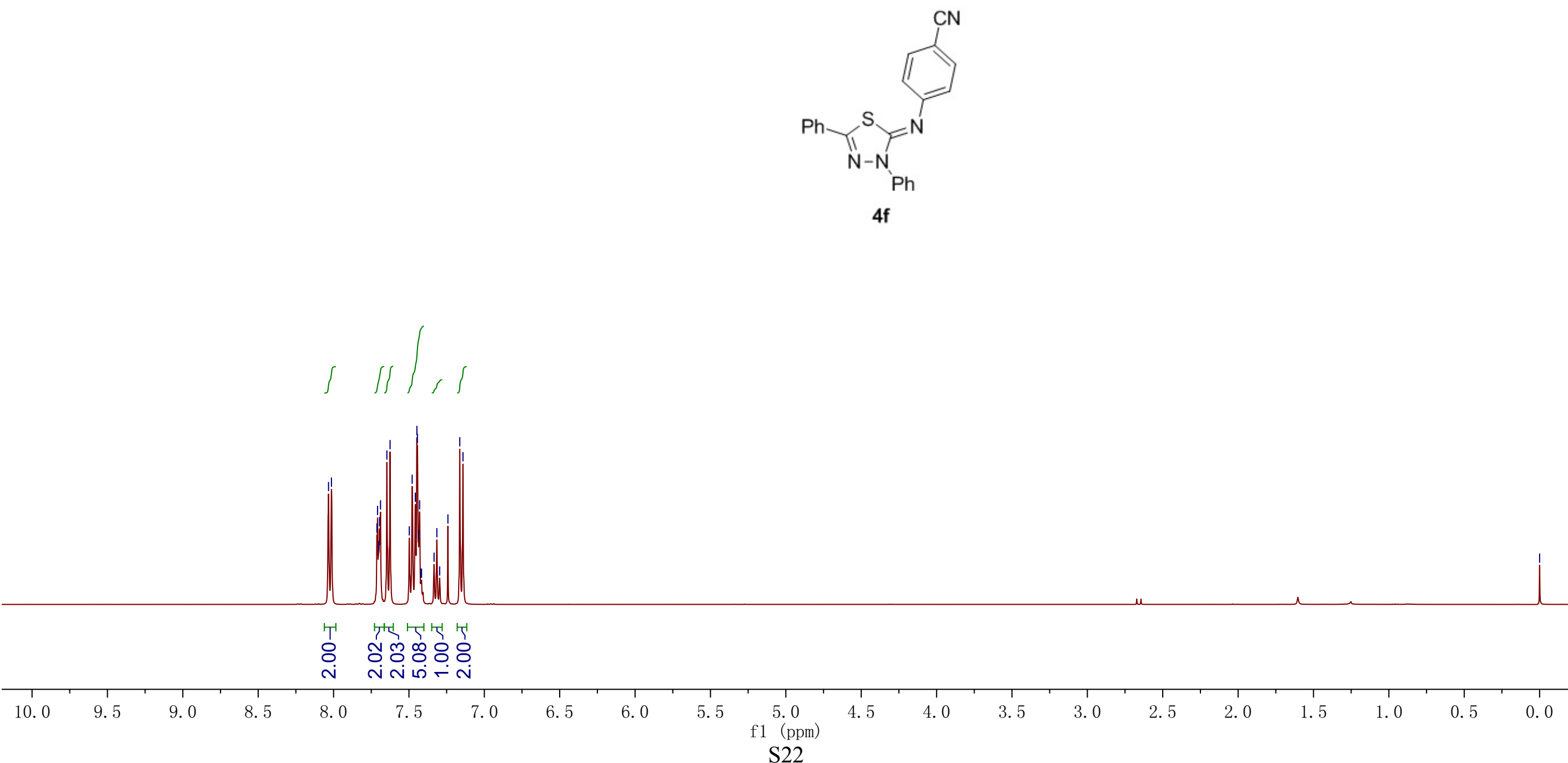
${ }^{13} \mathrm{C}$ NMR $\left(100 \mathrm{MHz}, \mathrm{CDCl}_{3}\right)$

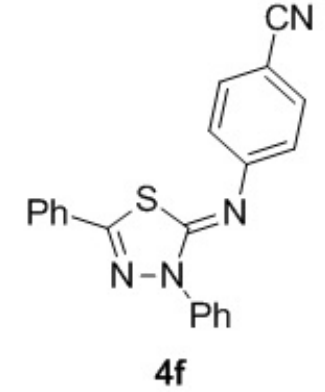

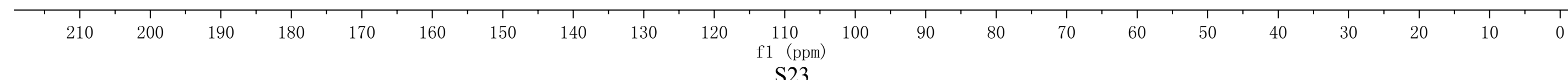


${ }^{1} \mathrm{H}$ NMR $\left(400 \mathrm{MHz}, \mathrm{CDCl}_{3}\right)$

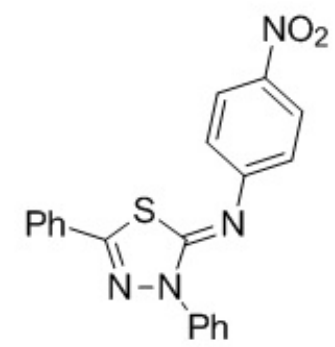

$4 \mathrm{~g}$

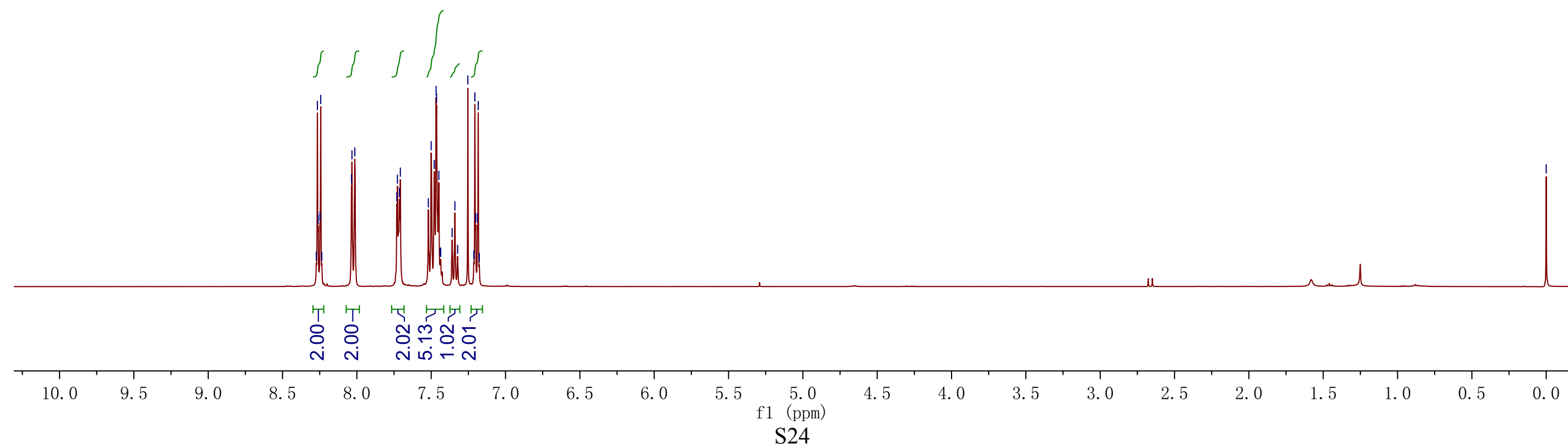


${ }^{13} \mathrm{C}$ NMR $\left(100 \mathrm{MHz}, \mathrm{CDCl}_{3}\right)$

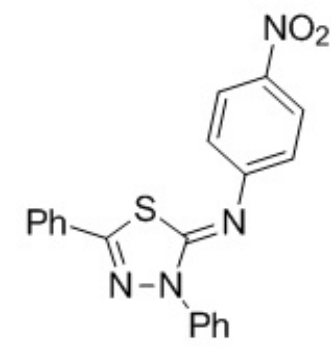

$4 \mathrm{~g}$
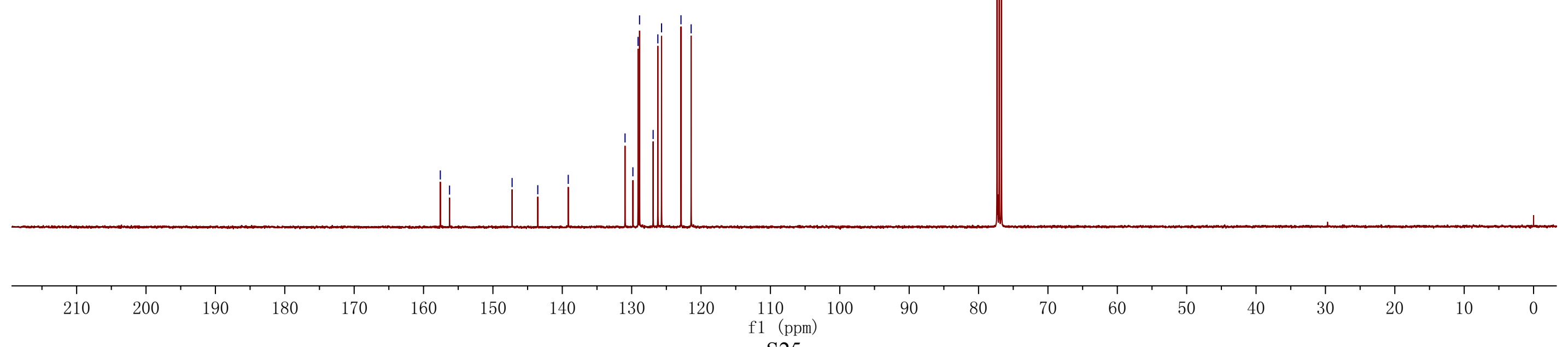
${ }^{1} \mathrm{H}$ NMR $\left(400 \mathrm{MHz}, \mathrm{CDCl}_{3}\right)$

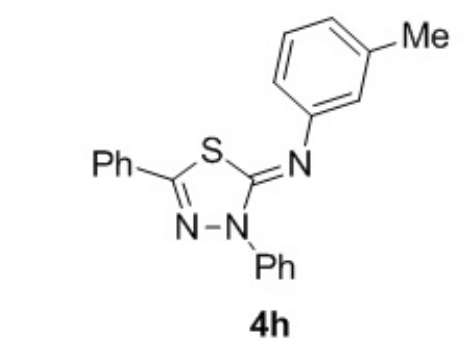

4h

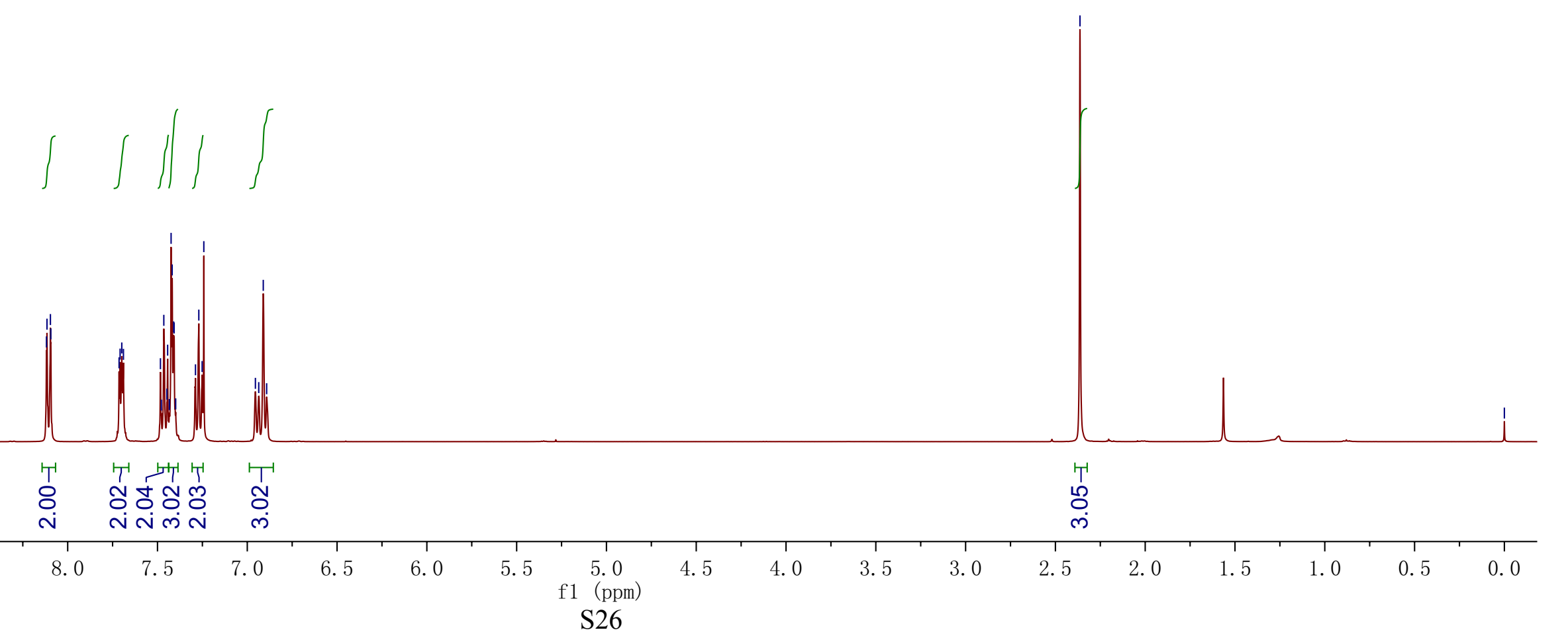


${ }^{13} \mathrm{C}$ NMR $\left(100 \mathrm{MHz}, \mathrm{CDCl}_{3}\right)$
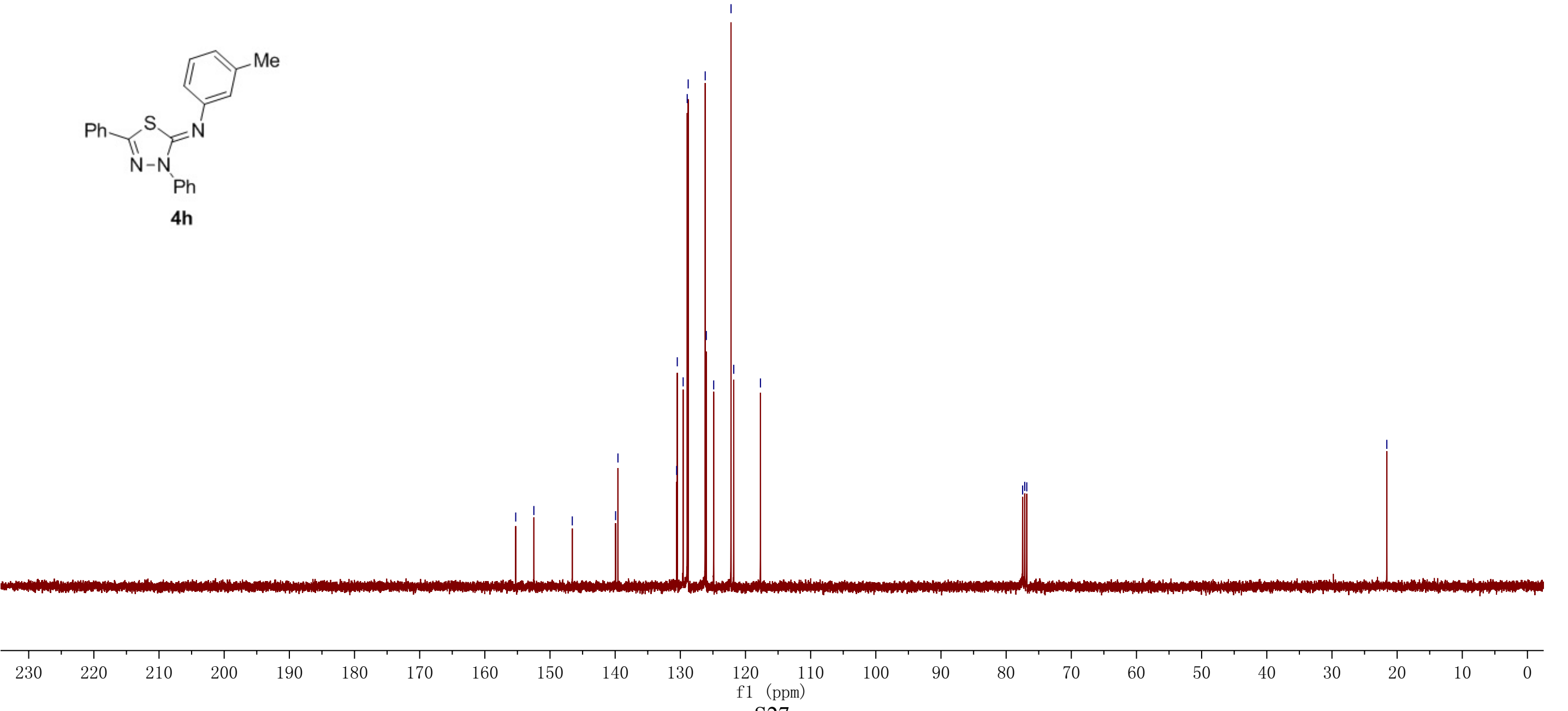
${ }^{1} \mathrm{H}$ NMR $\left(400 \mathrm{MHz}, \mathrm{CDCl}_{3}\right)$

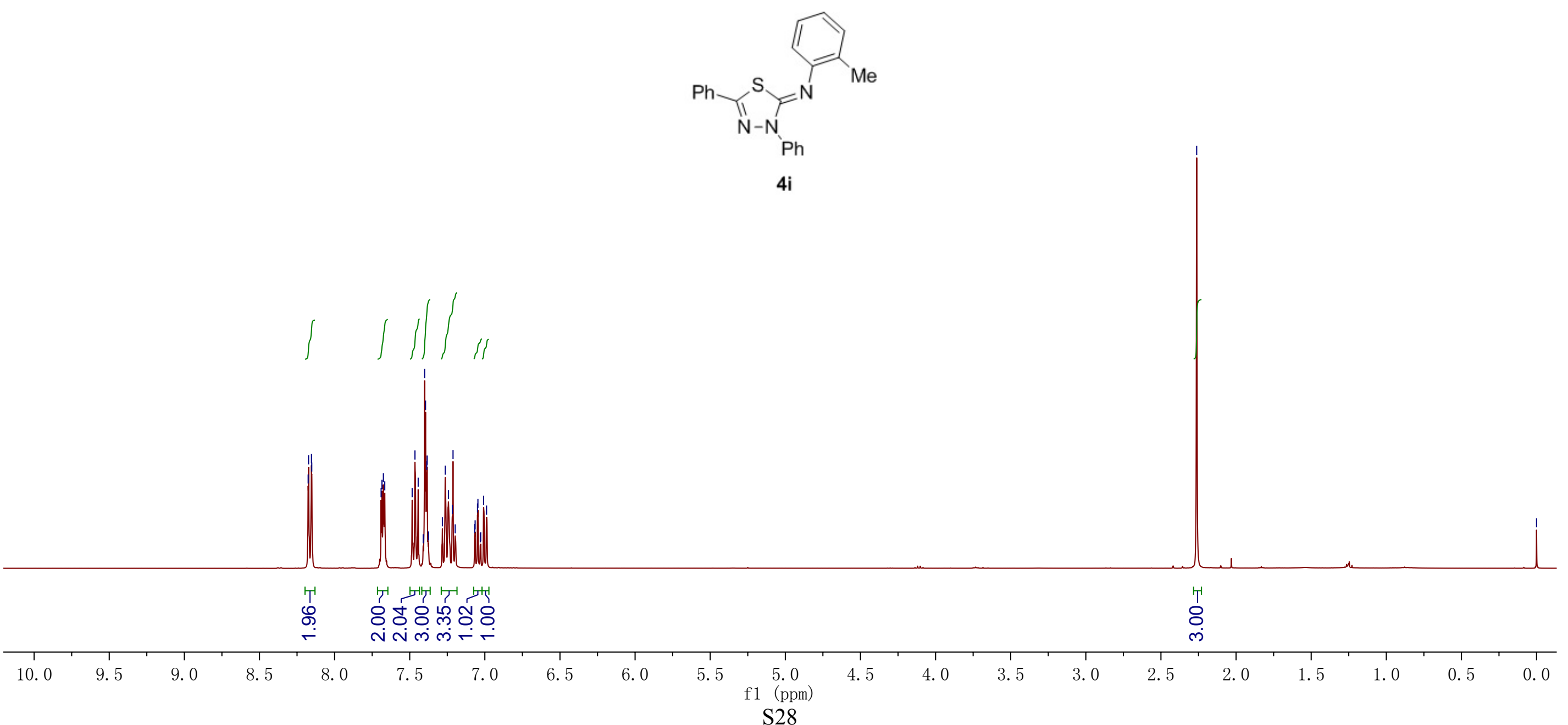


${ }^{13} \mathrm{C}$ NMR $\left(100 \mathrm{MHz}, \mathrm{CDCl}_{3}\right)$
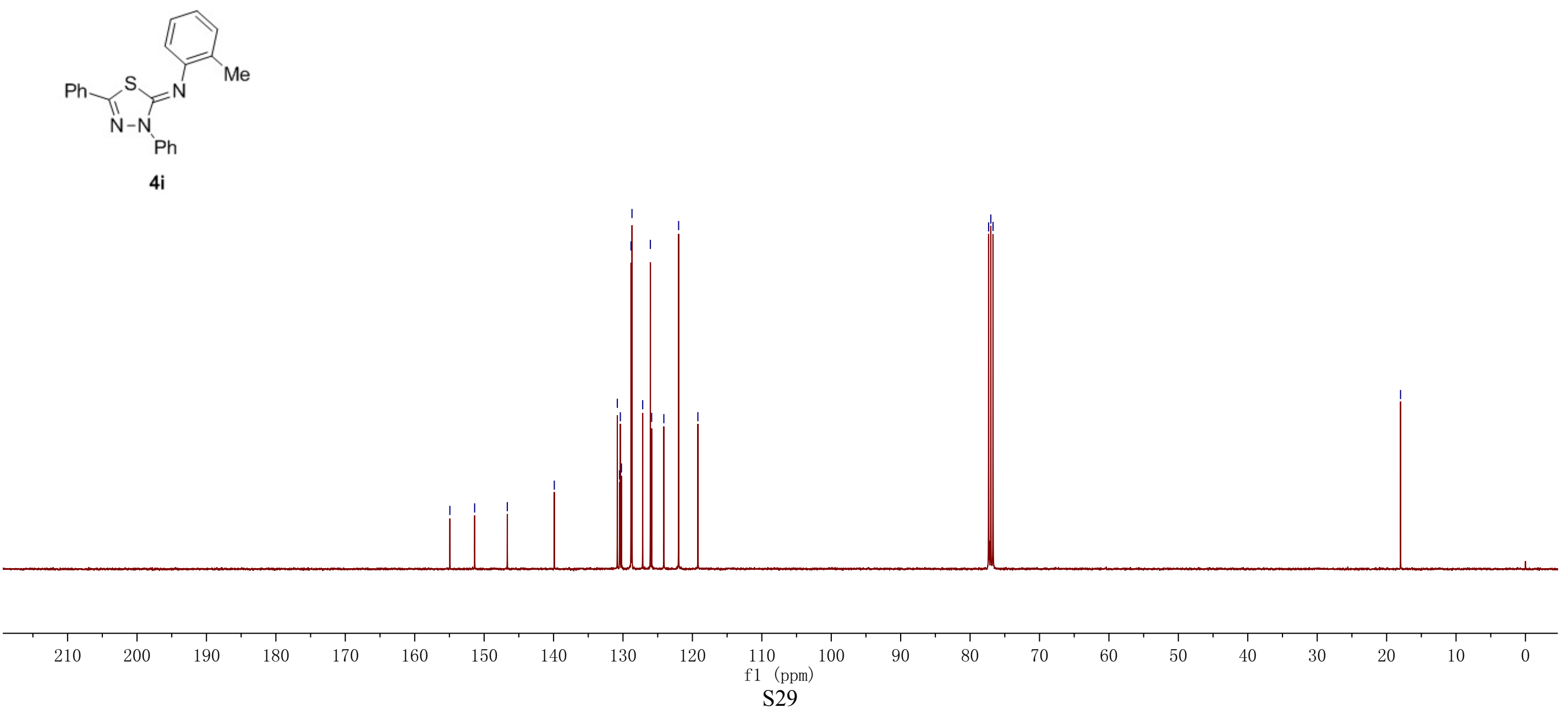


\section{${ }^{1} \mathrm{H}$ NMR $\left(400 \mathrm{MHz}, \mathrm{CDCl}_{3}\right)$}
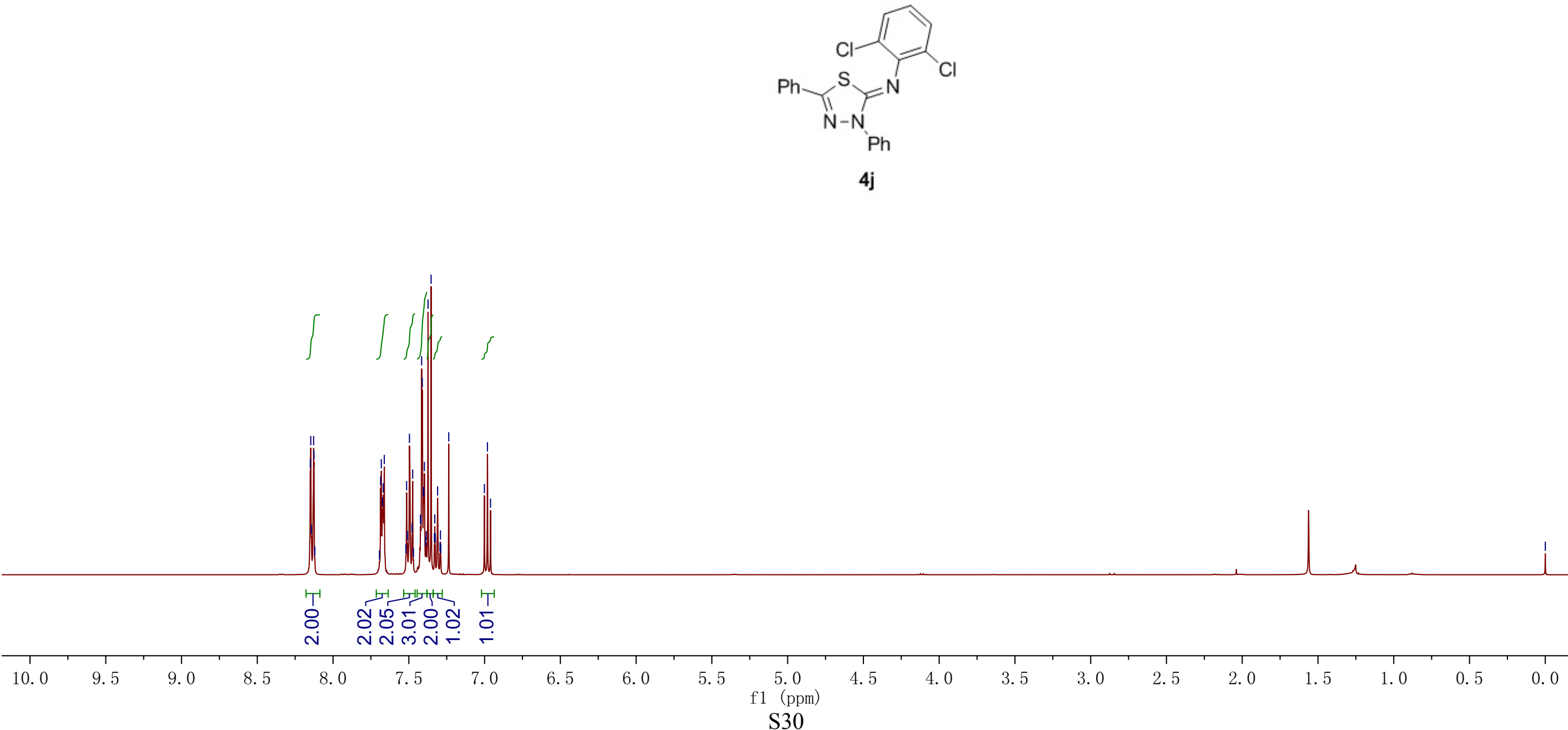


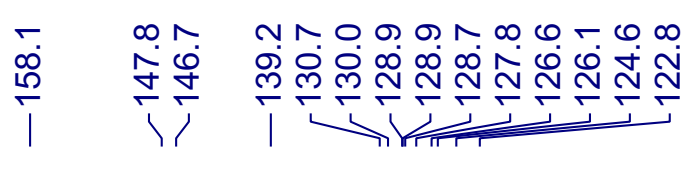

${ }^{13} \mathrm{C} \mathrm{NMR}\left(100 \mathrm{MHz}, \mathrm{CDCl}_{3}\right)$

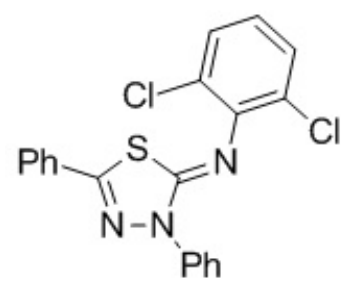

4j
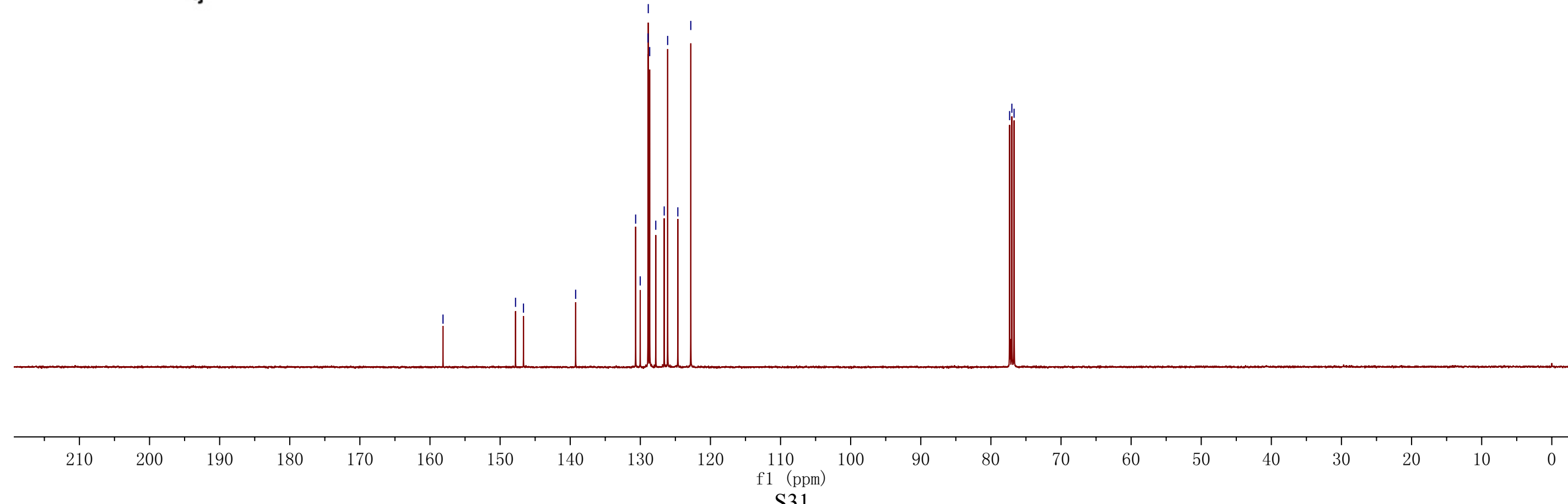
${ }^{1} \mathrm{H}$ NMR (400 MHz, $\mathrm{CDCl}_{3}$ )
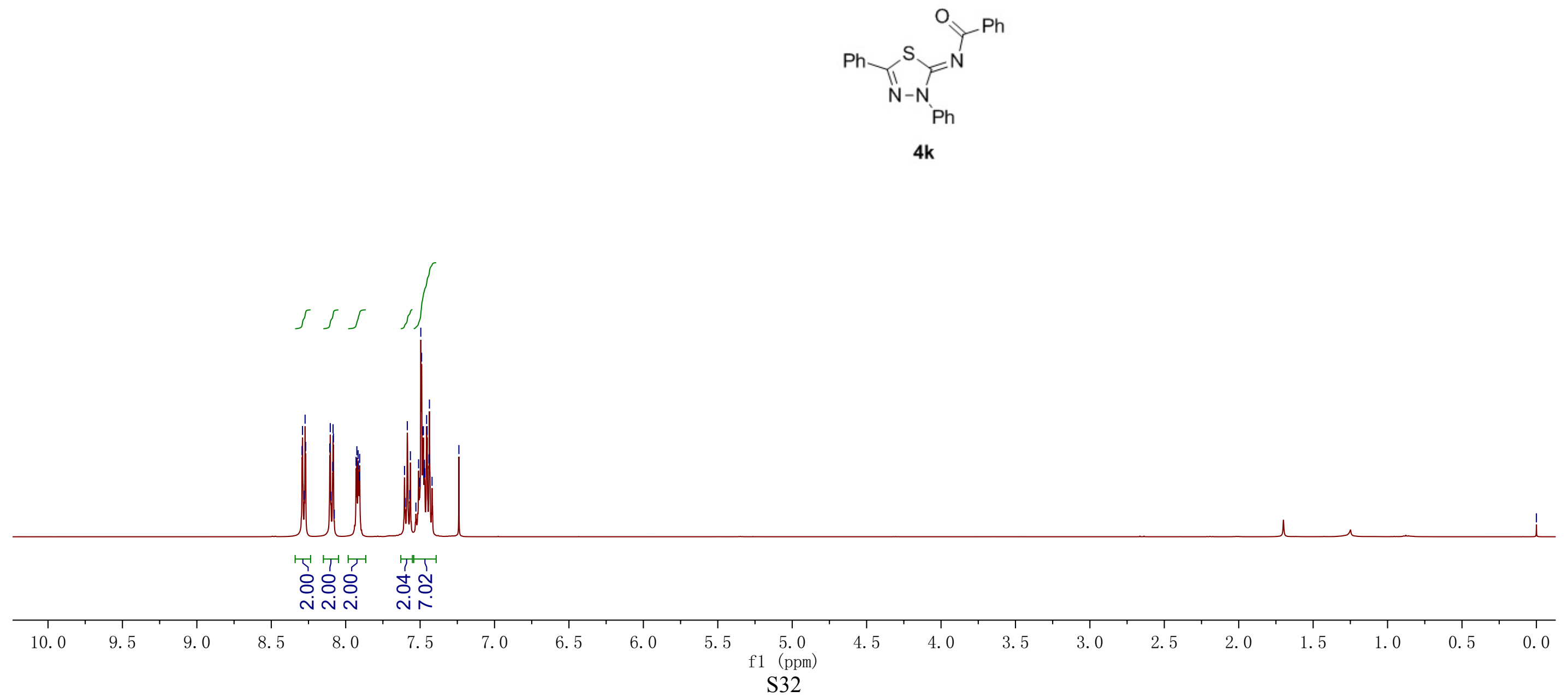
${ }^{13} \mathrm{C}$ NMR $\left(100 \mathrm{MHz}, \mathrm{CDCl}_{3}\right)$

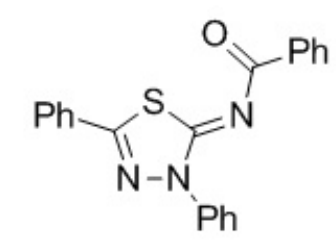

$4 k$

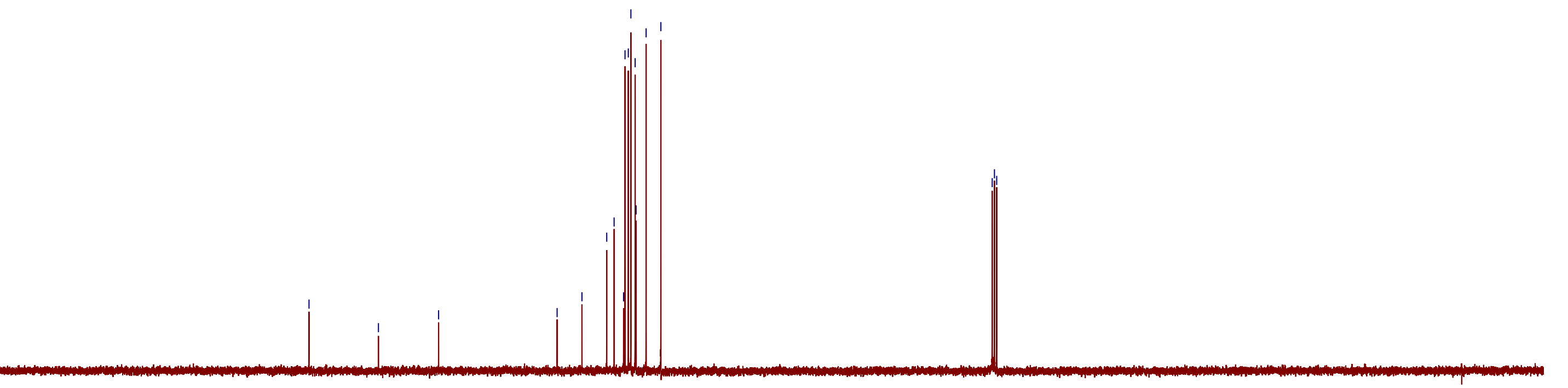

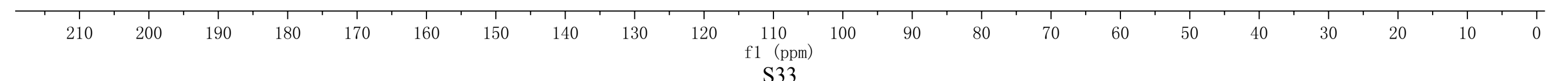


${ }^{1} \mathrm{H}$ NMR (400 MHz, $\mathrm{CDCl}_{3}$ )

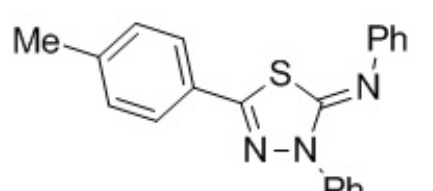

$4 \mathrm{~m}$
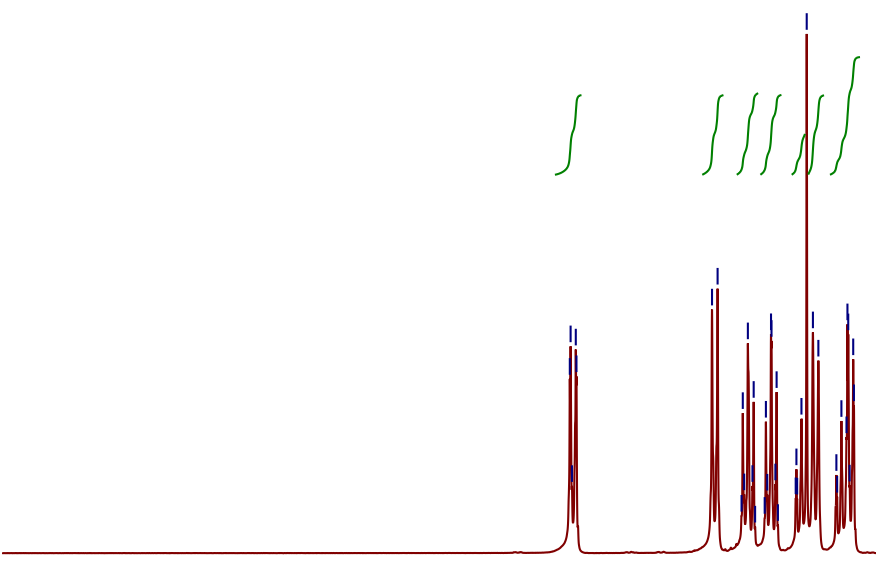

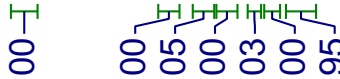

n hing nis

$8,0+7,5,7,0$

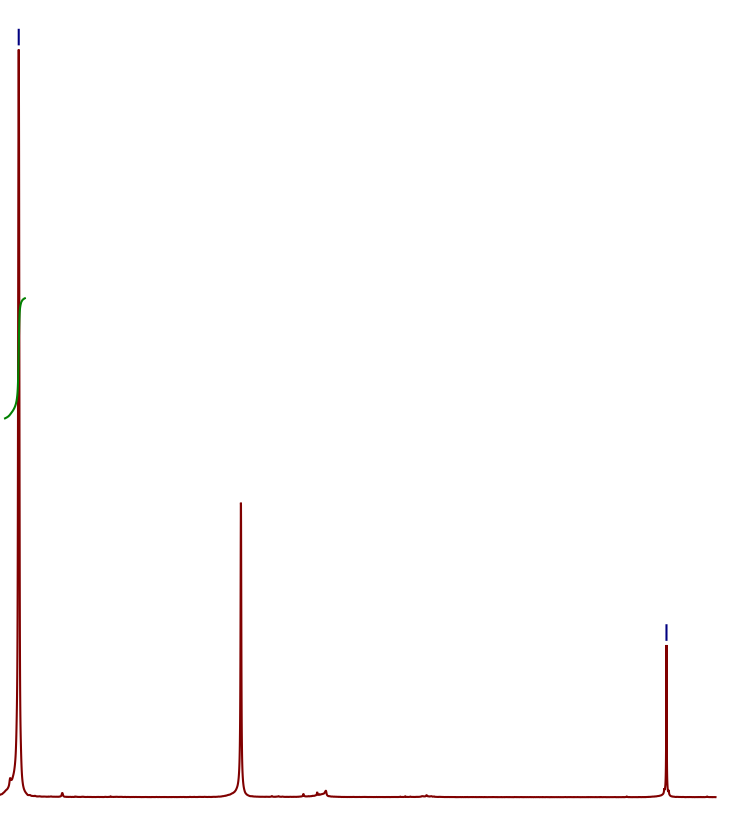


${ }^{13} \mathrm{C}$ NMR (100 MHz, $\left.\mathrm{CDCl}_{3}\right)$

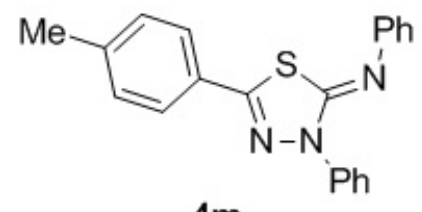

$4 m$

$\mathrm{Ph}$
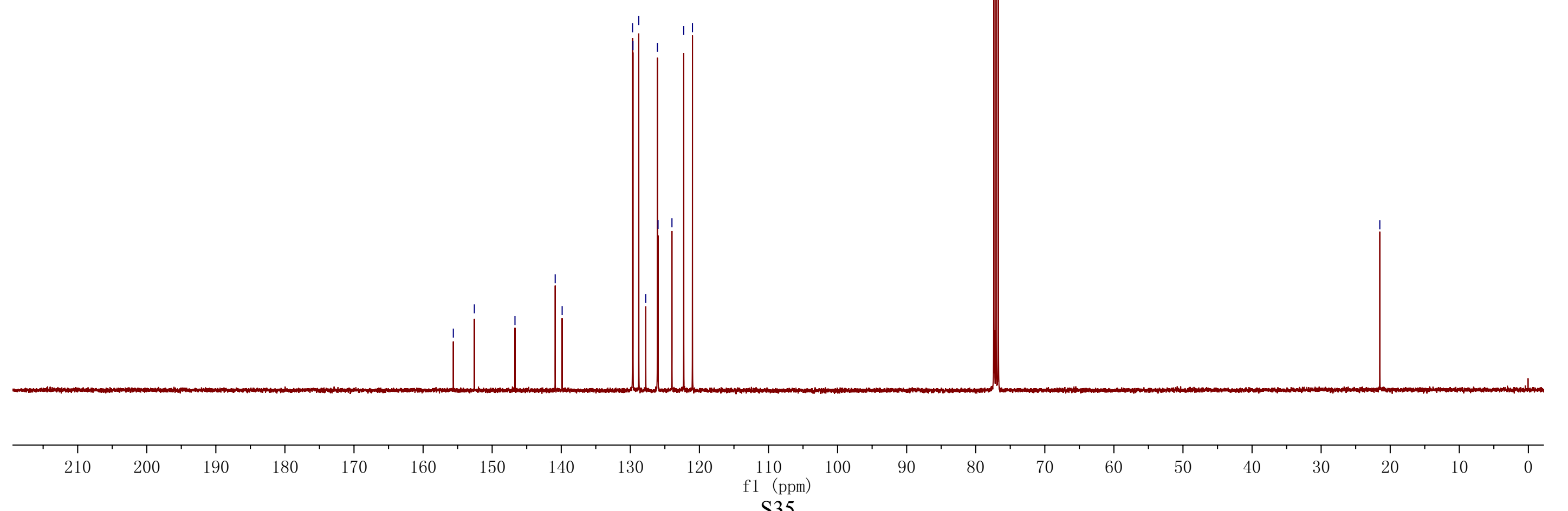
${ }^{1} \mathrm{H}$ NMR $\left(400 \mathrm{MHz}, \mathrm{CDCl}_{3}\right)$

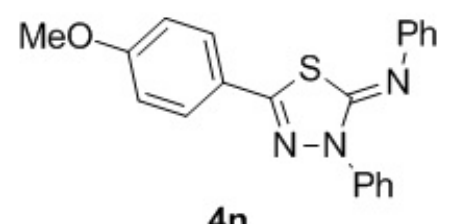

$4 n$
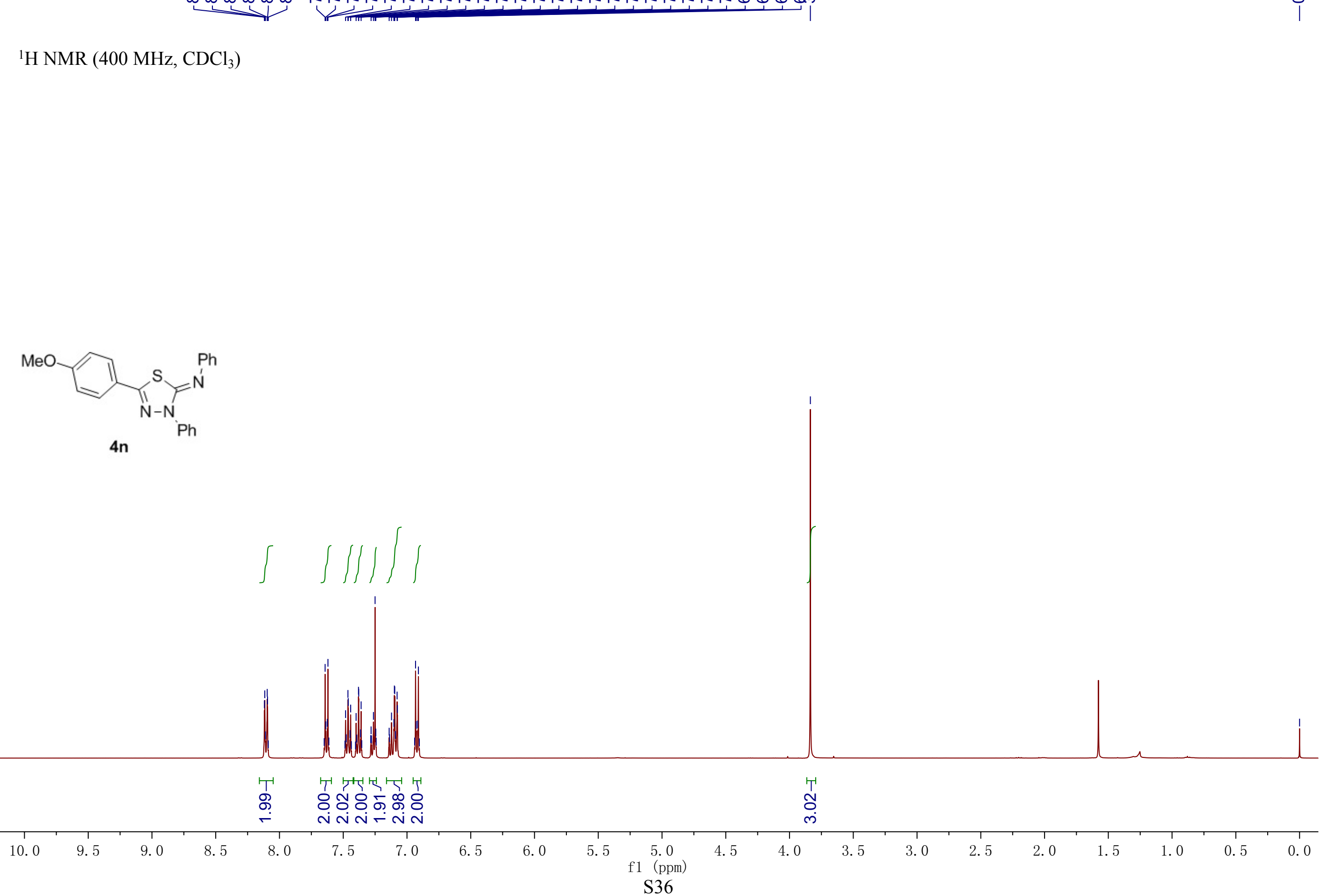
${ }^{13} \mathrm{C}$ NMR (100 MHz, $\left.\mathrm{CDCl}_{3}\right)$

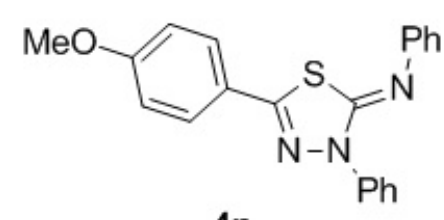

$4 n$

$\mathrm{Ph}$

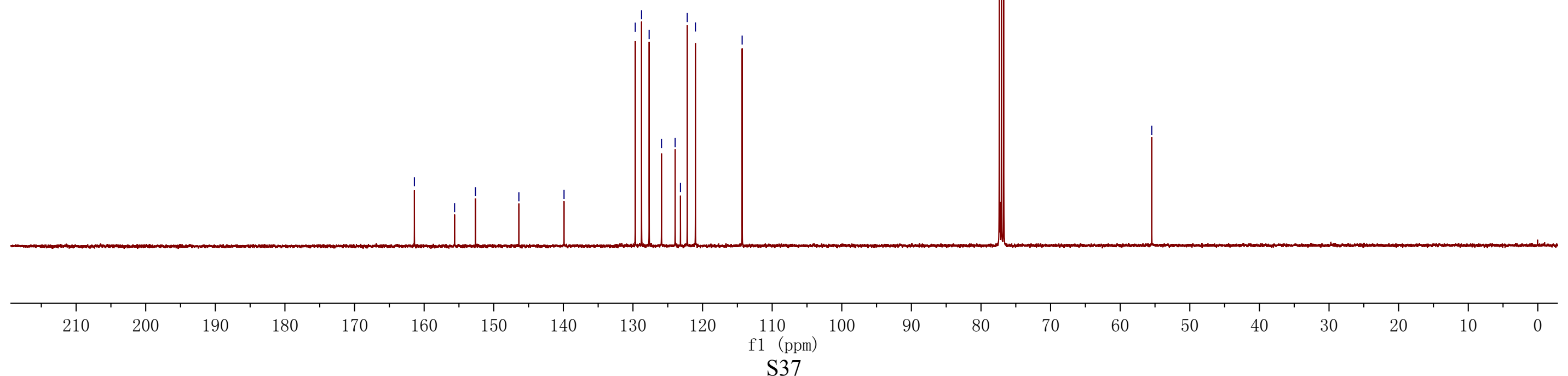


${ }^{1} \mathrm{H}$ NMR $\left(400 \mathrm{MHz}, \mathrm{CDCl}_{3}\right)$

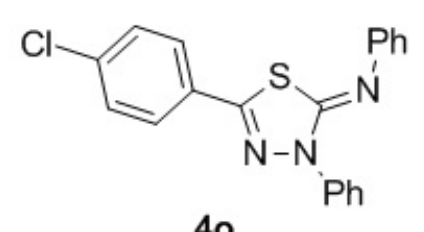

40

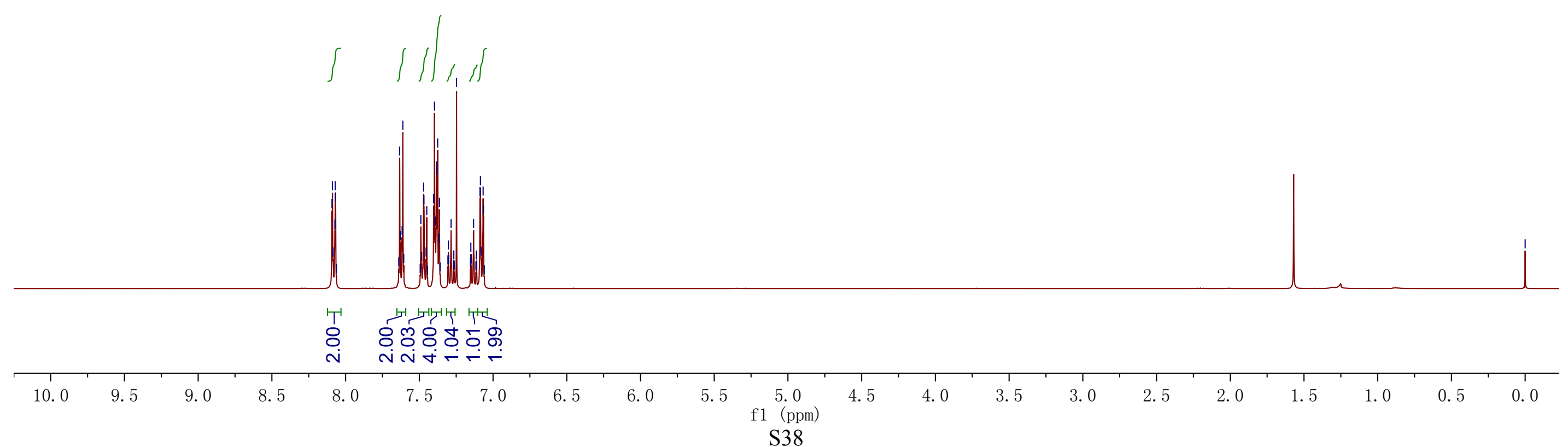


${ }^{13} \mathrm{C}$ NMR $\left(100 \mathrm{MHz}, \mathrm{CDCl}_{3}\right)$

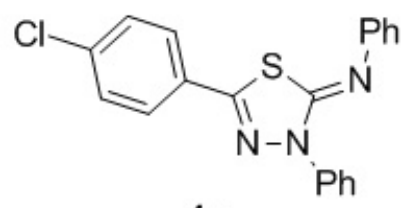

40

$\mathrm{Ph}$
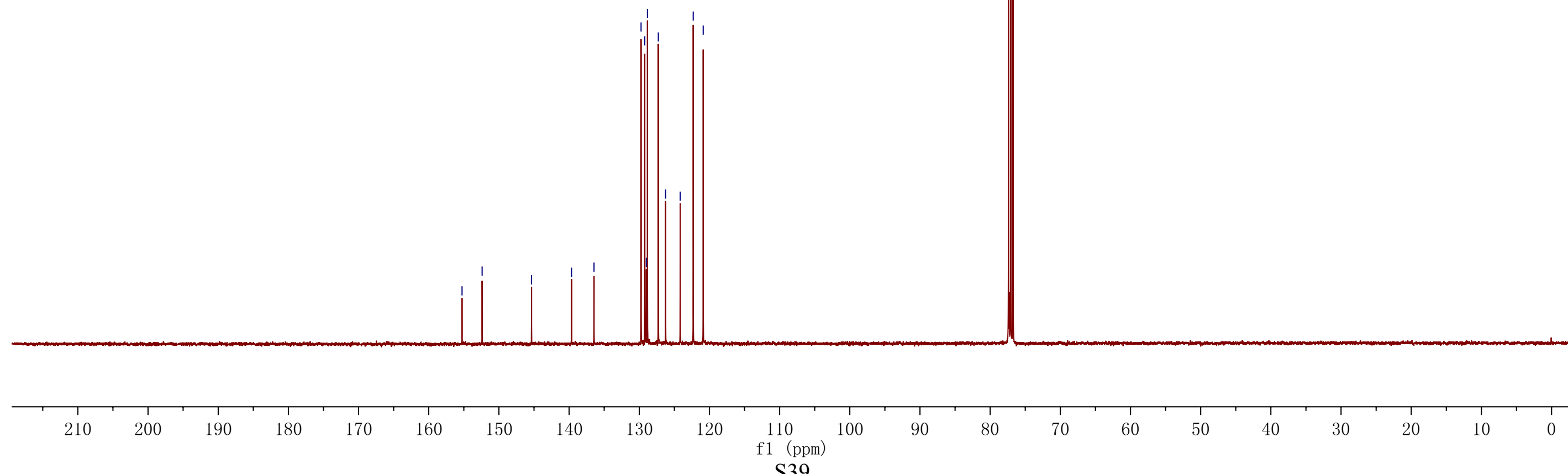
${ }^{1} \mathrm{H}$ NMR (400 MHz, $\mathrm{CDCl}_{3}$ )

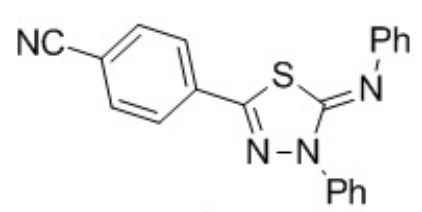

$4 p$

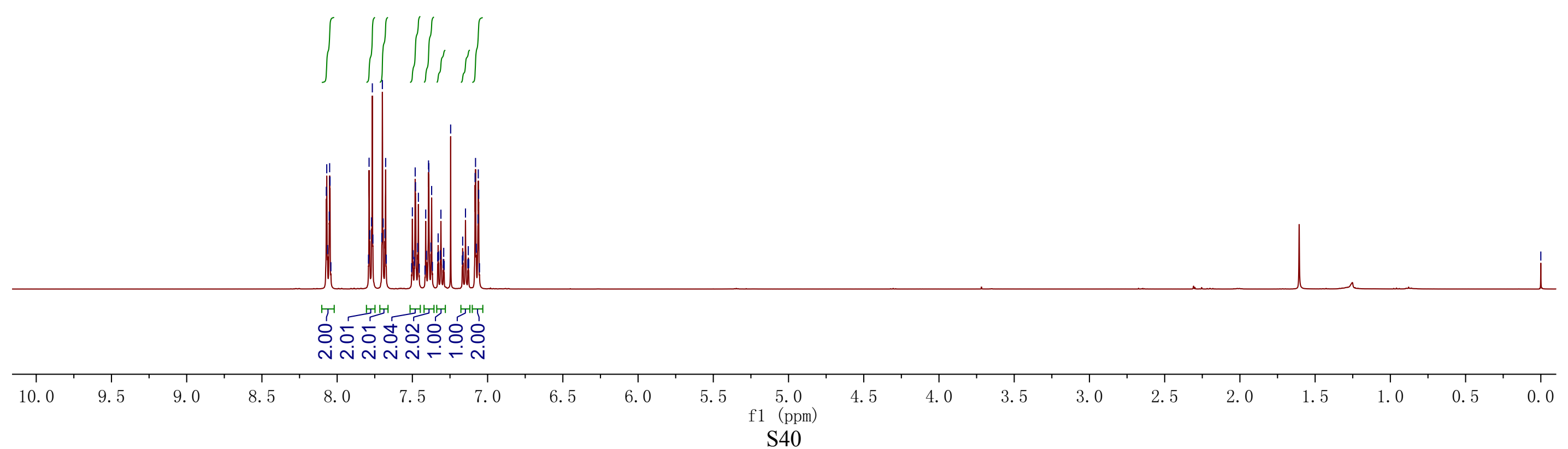


${ }^{13} \mathrm{C}$ NMR (100 MHz, $\left.\mathrm{CDCl}_{3}\right)$

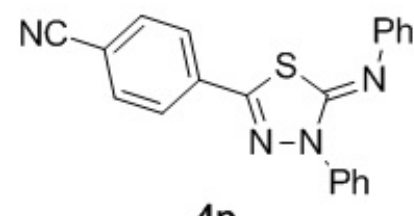

$4 p$

$$
\text { Ph }
$$

$$
\text { (n) }
$$
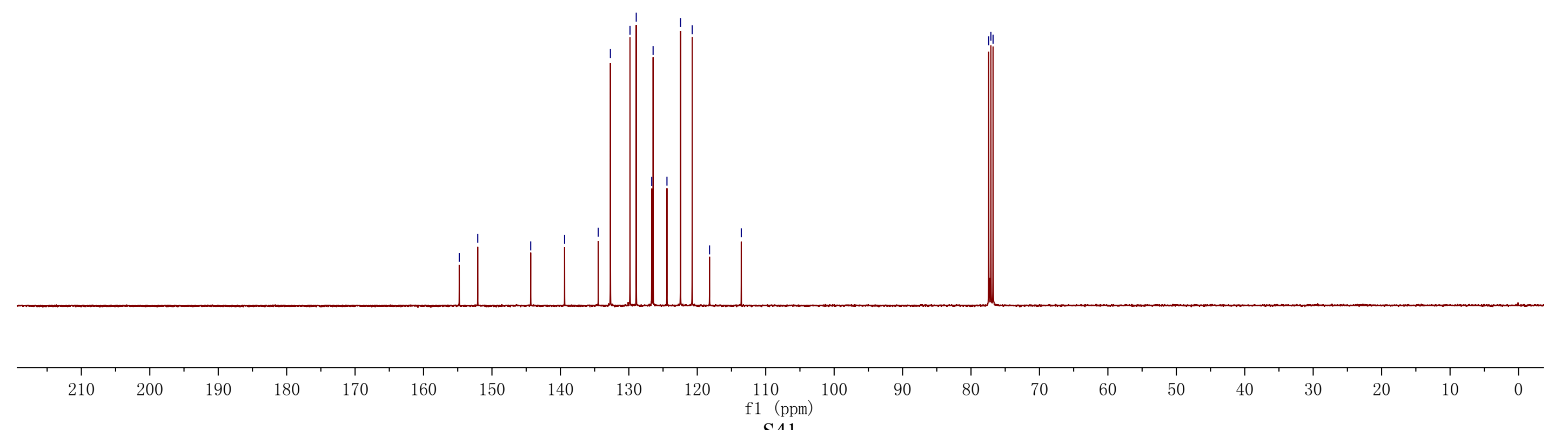

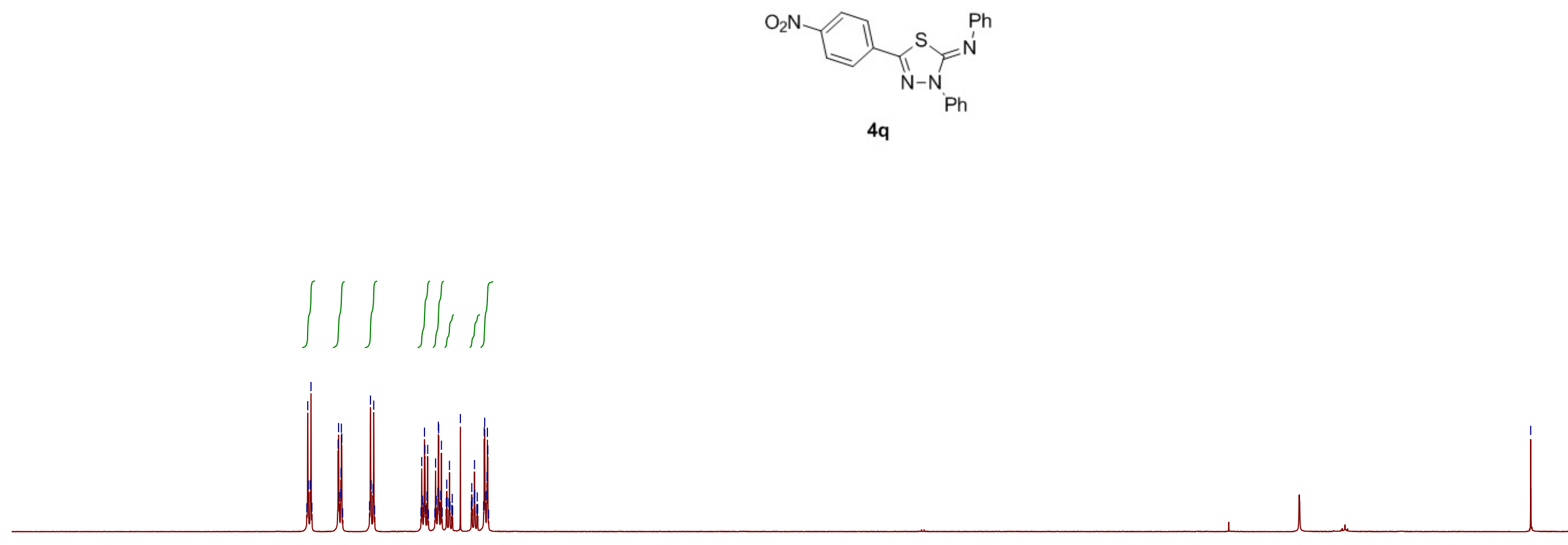

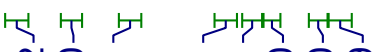

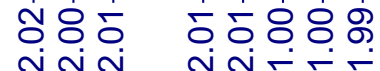

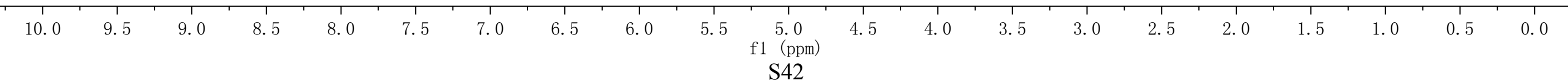


${ }^{13} \mathrm{C}$ NMR (100 MHz, $\left.\mathrm{CDCl}_{3}\right)$

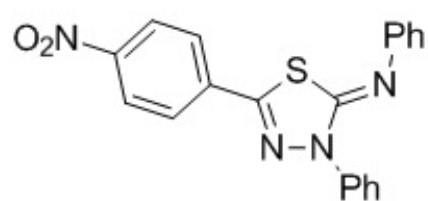

$4 q$

$\mathrm{Ph}$

$\mathrm{Ph}$

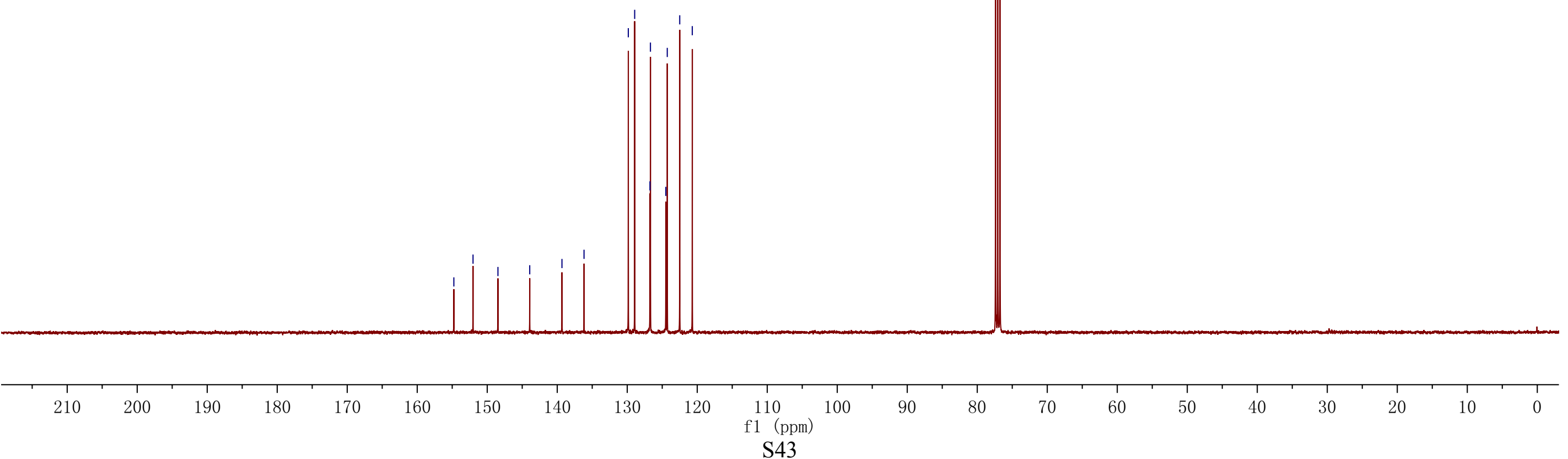




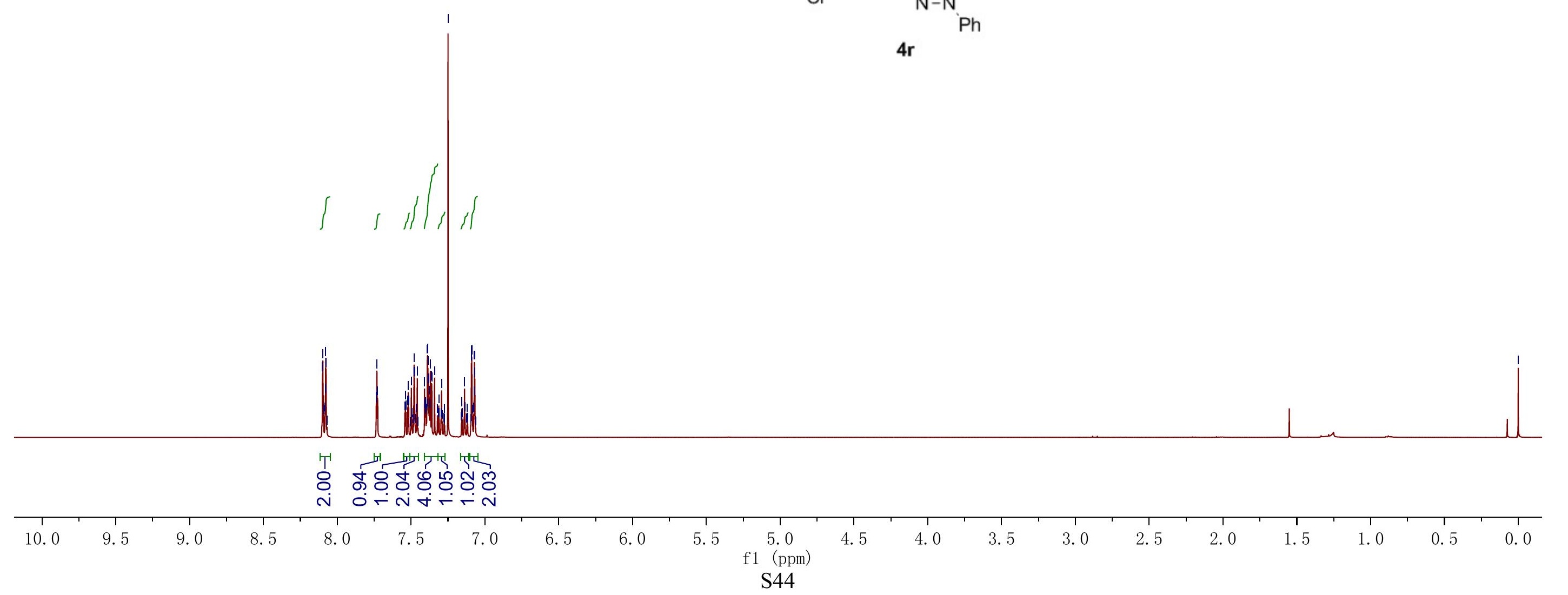


${ }^{13} \mathrm{C}$ NMR (100 MHz, $\left.\mathrm{CDCl}_{3}\right)$

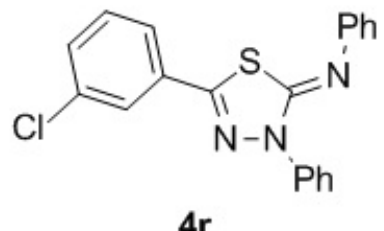

\section{h}

(n)

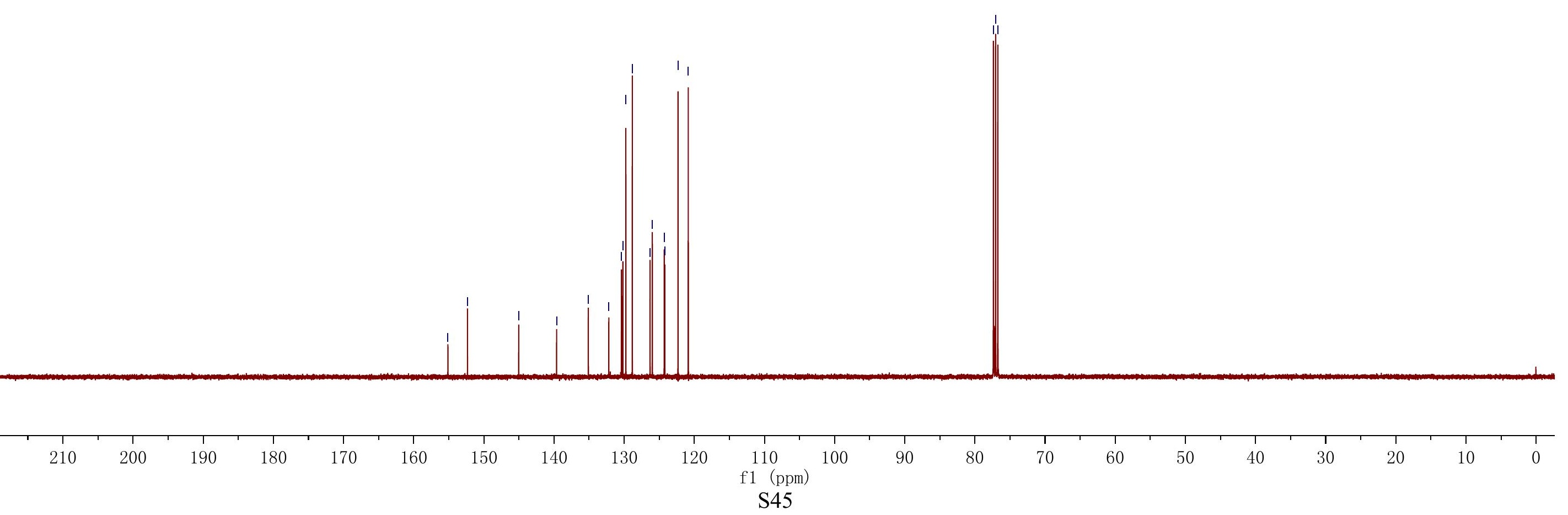


${ }^{1} \mathrm{H}$ NMR (400 MHz, $\mathrm{CDCl}_{3}$ )
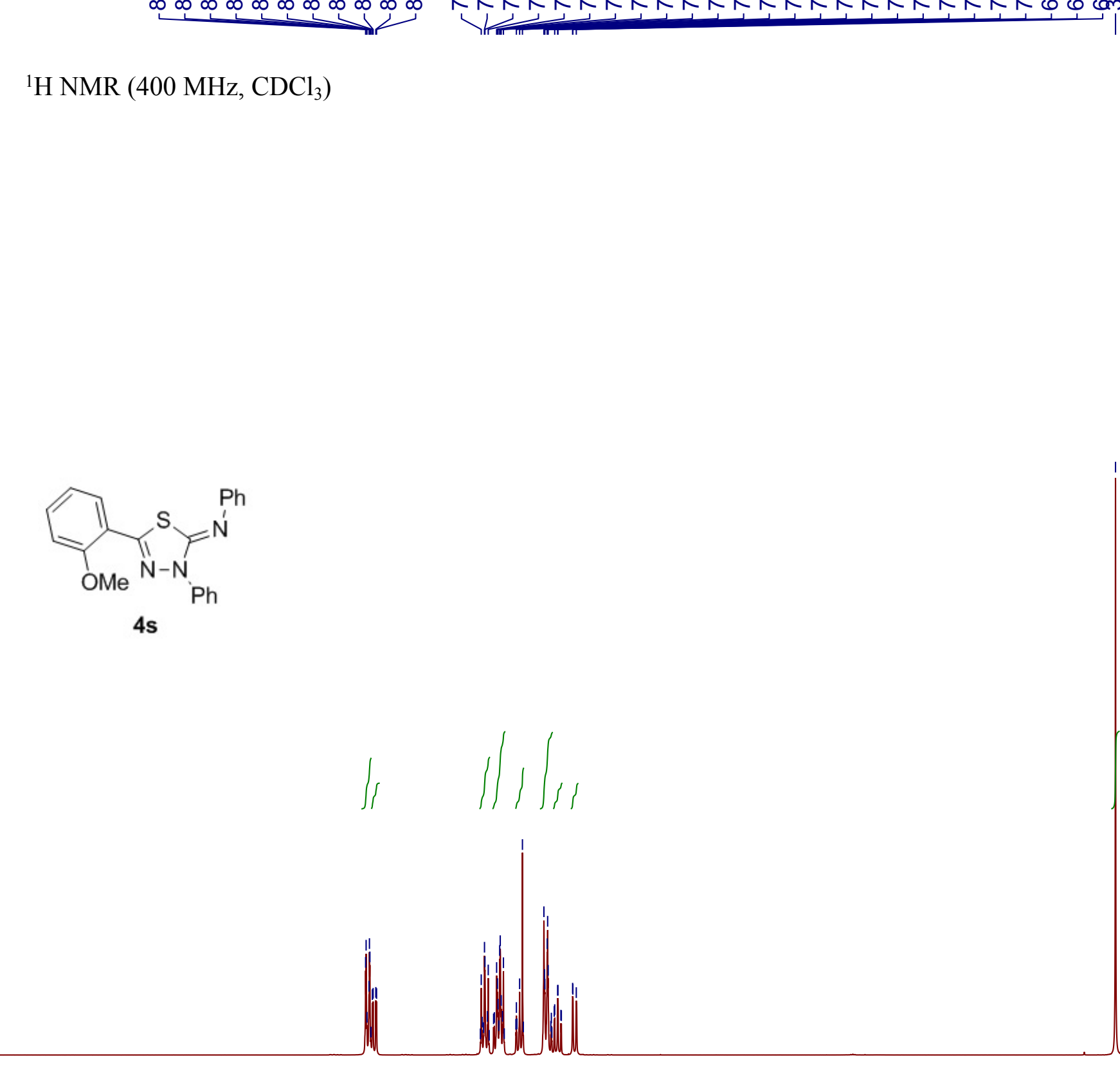

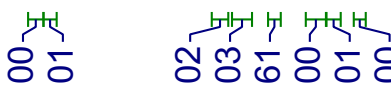

N

$9.5 \quad 9.0 \quad 8.5$

$8.0 \quad 7.5$

$7.0 \quad 6.5$

6.0

5.5 
${ }^{13} \mathrm{C}$ NMR (100 MHz, $\left.\mathrm{CDCl}_{3}\right)$

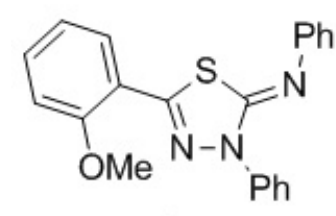

$4 \mathrm{~s}$
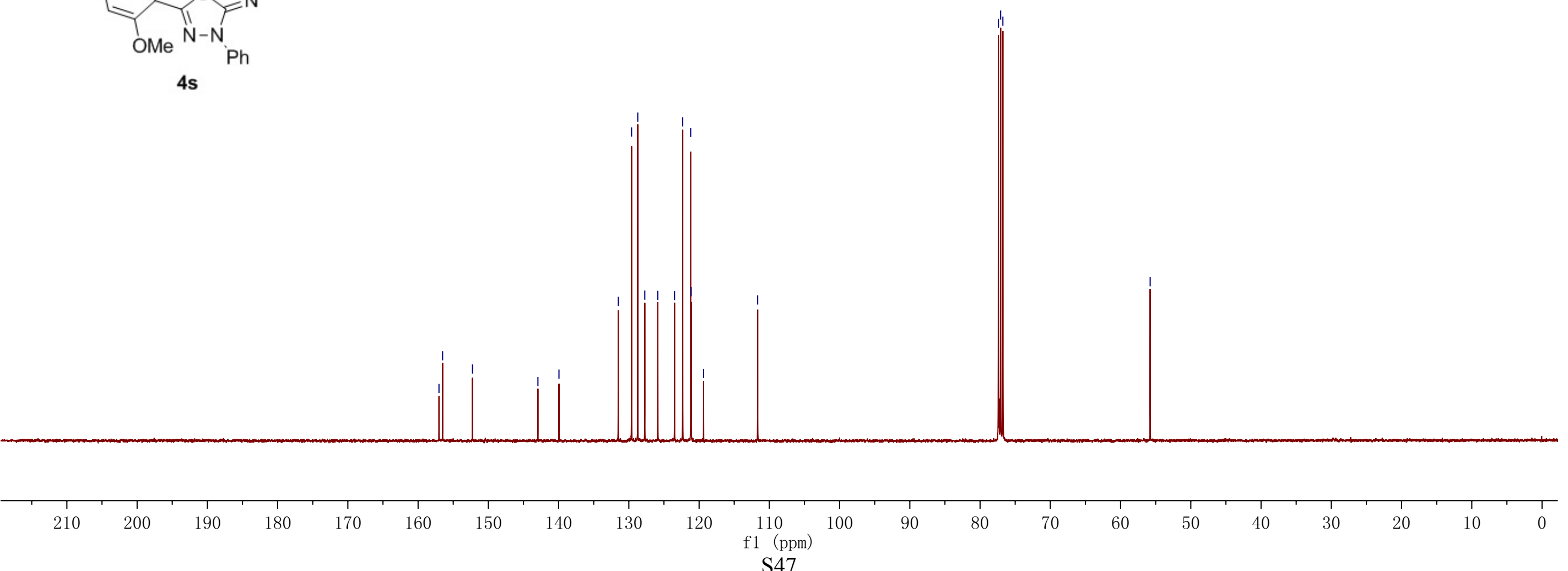
${ }^{1} \mathrm{H} \mathrm{NMR}\left(400 \mathrm{MHz}, \mathrm{CDCl}_{3}\right)$

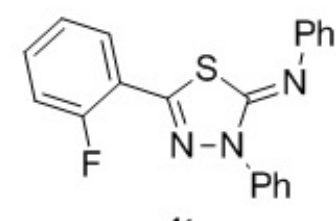

4t

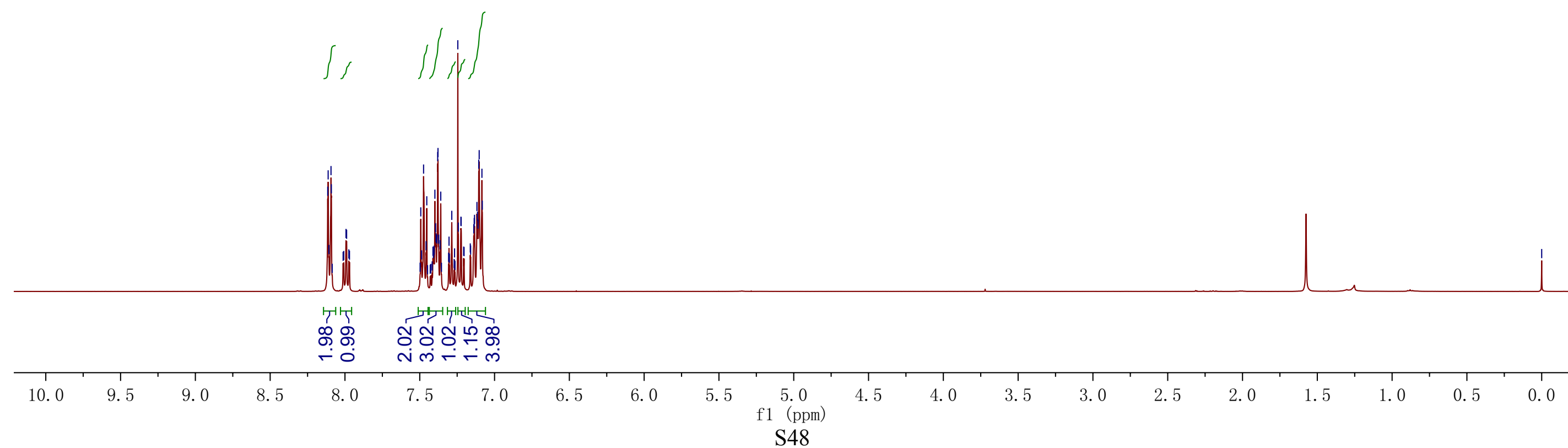


${ }^{13} \mathrm{C}$ NMR (100 MHz, $\mathrm{CDCl}_{3}$ )

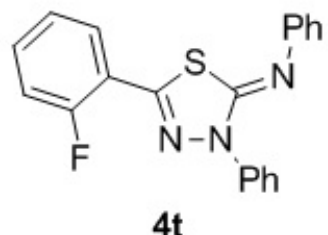

$4 t$
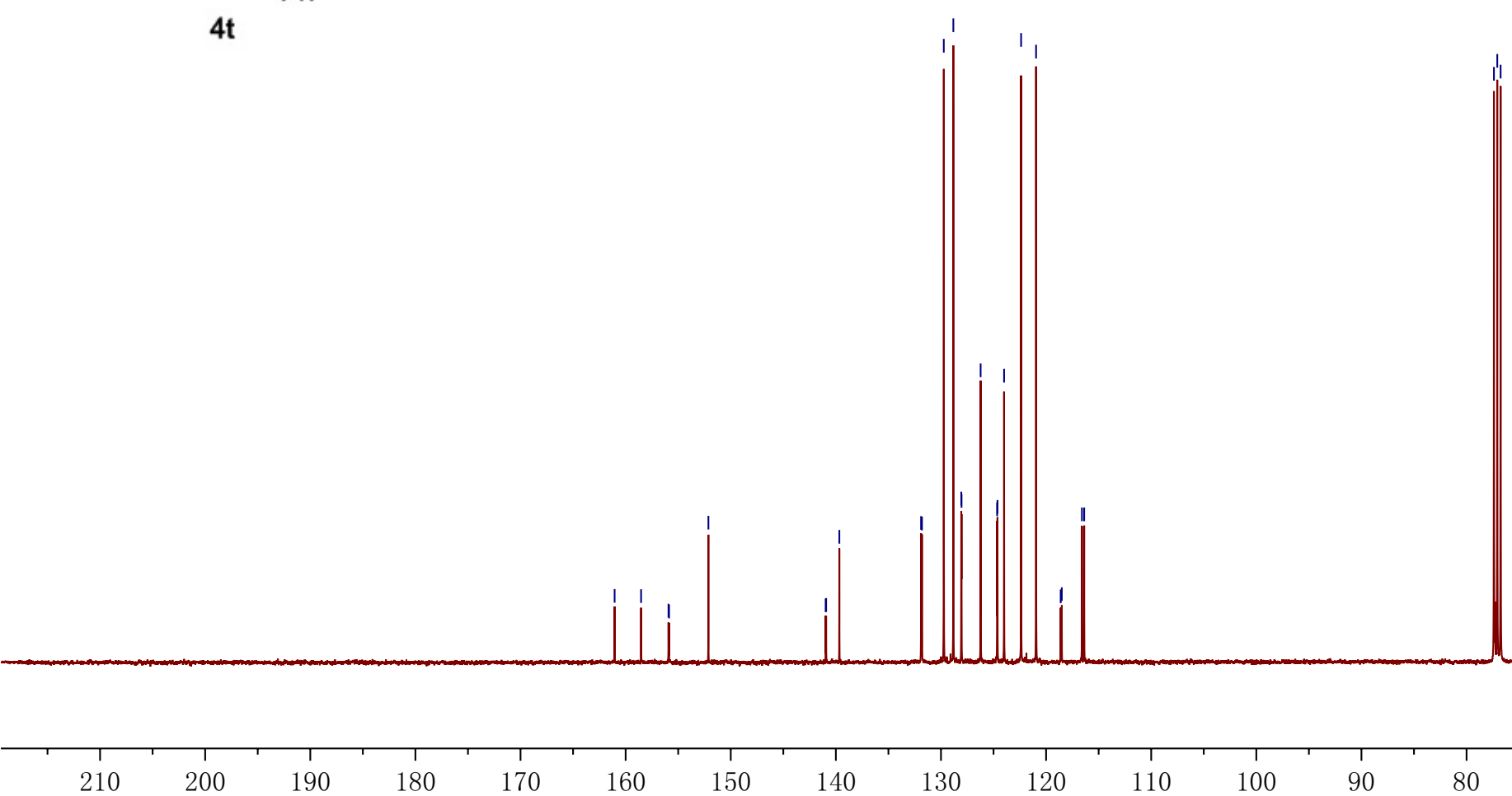

$200 \quad 190$

180

170

160

150

$140 \quad 130$

120

$110 \quad 100$

90

80

70

50 S49 
${ }^{1} \mathrm{H} \mathrm{NMR}\left(400 \mathrm{MHz}, \mathrm{CDCl}_{3}\right)$

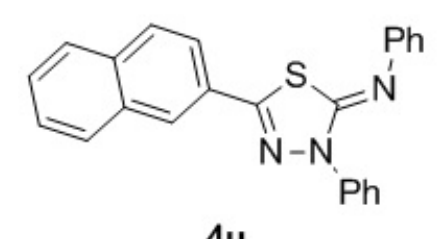

$4 \mathbf{u}$
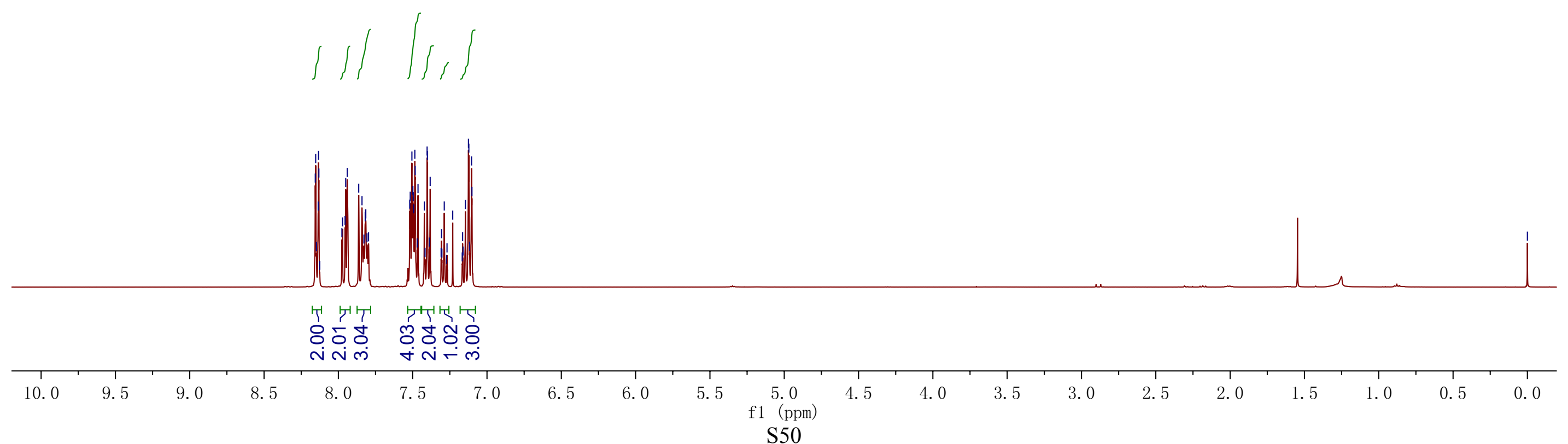
${ }^{13} \mathrm{C}$ NMR $\left(100 \mathrm{MHz}, \mathrm{CDCl}_{3}\right)$

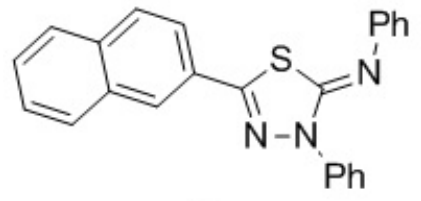

$4 \mathrm{u}$ 
${ }^{1} \mathrm{H}$ NMR (400 MHz, $\mathrm{CDCl}_{3}$ )

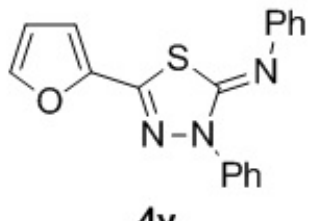

$4 v$

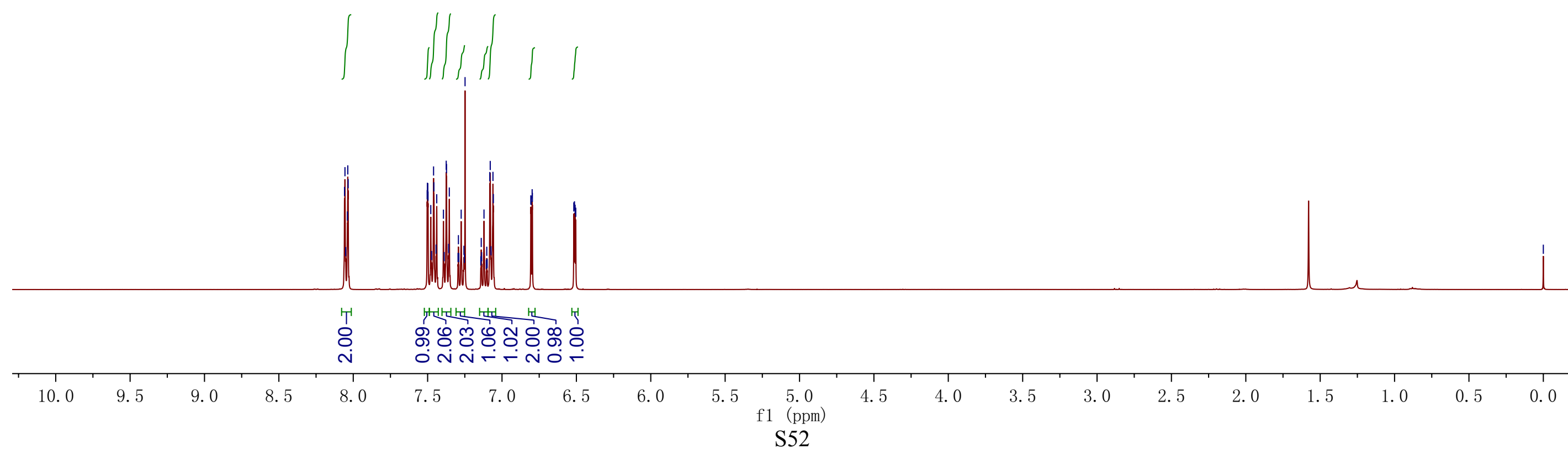


${ }^{13} \mathrm{C}$ NMR $\left(100 \mathrm{MHz}, \mathrm{CDCl}_{3}\right)$
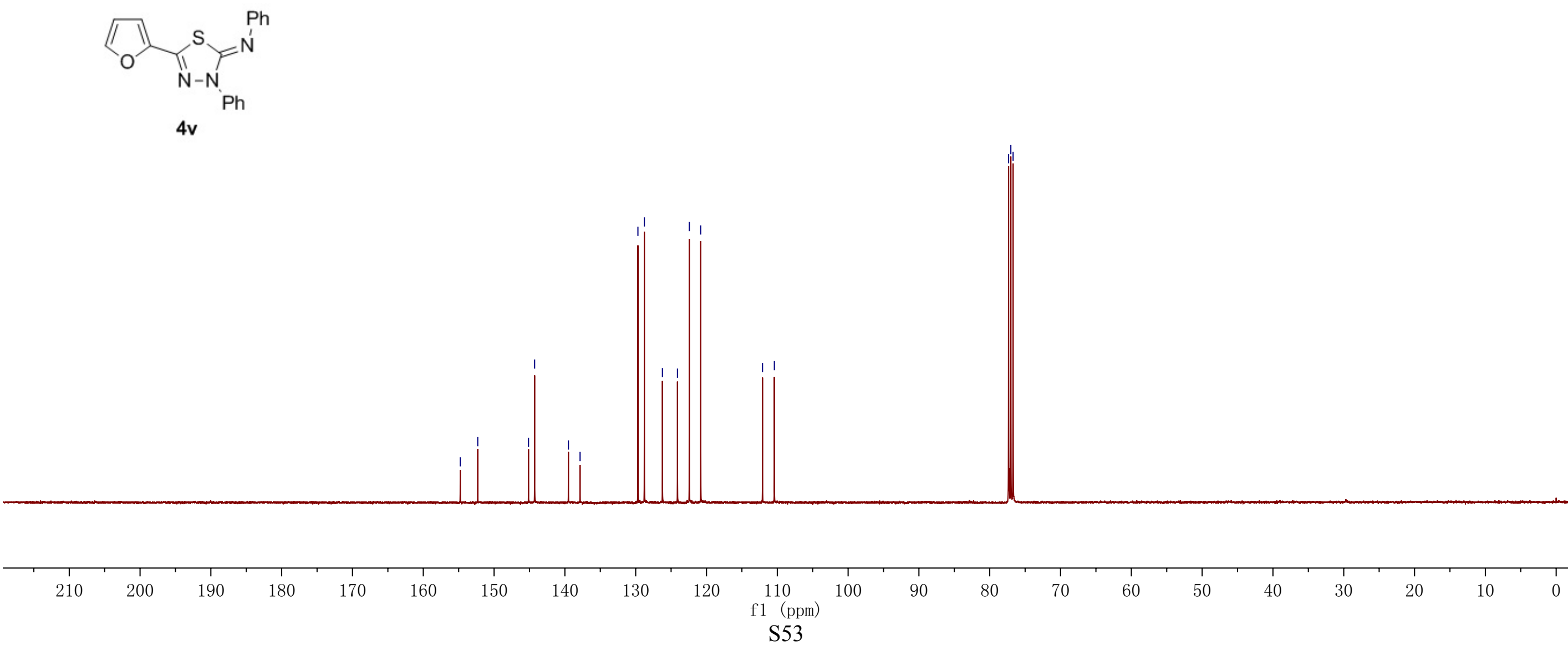
${ }^{1} \mathrm{H}$ NMR $\left(400 \mathrm{MHz}, \mathrm{CDCl}_{3}\right)$

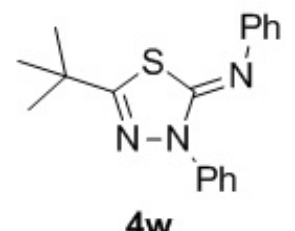

$4 w$

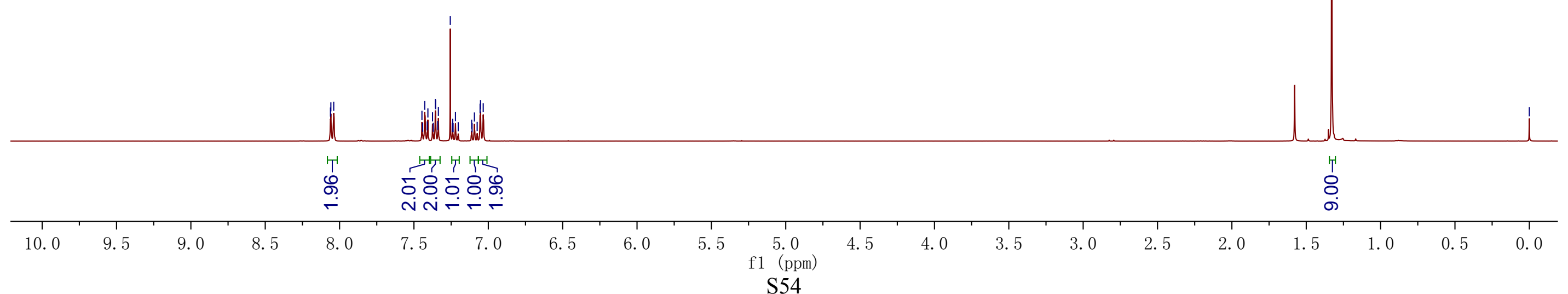


${ }^{13} \mathrm{C}$ NMR (100 MHz, $\left.\mathrm{CDCl}_{3}\right)$

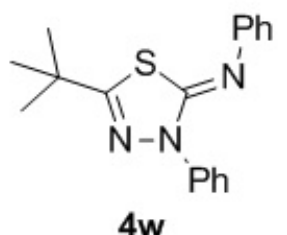

$$
\mathrm{Ph}
$$

w

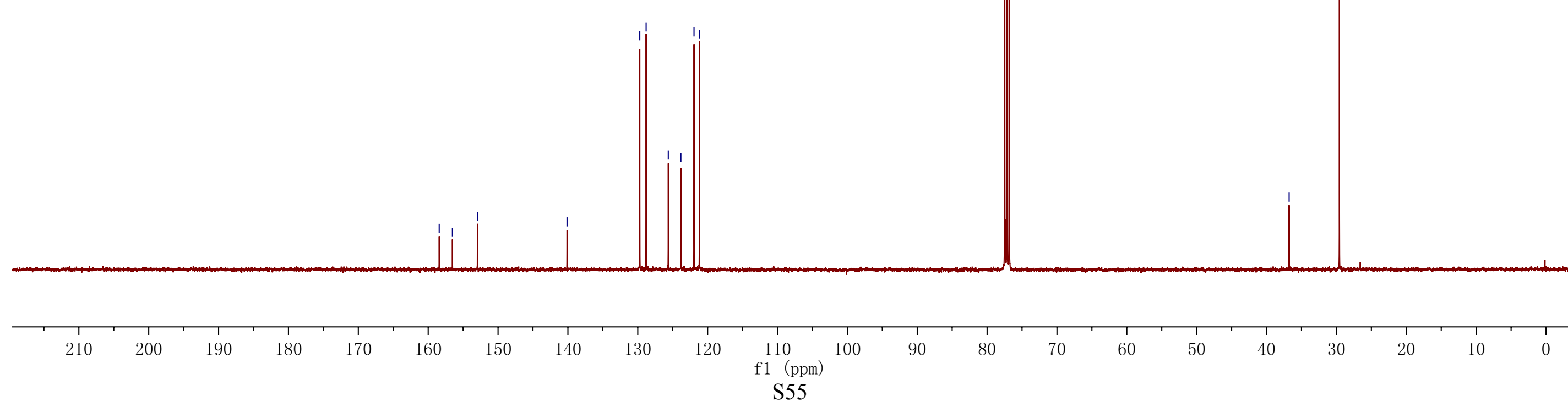




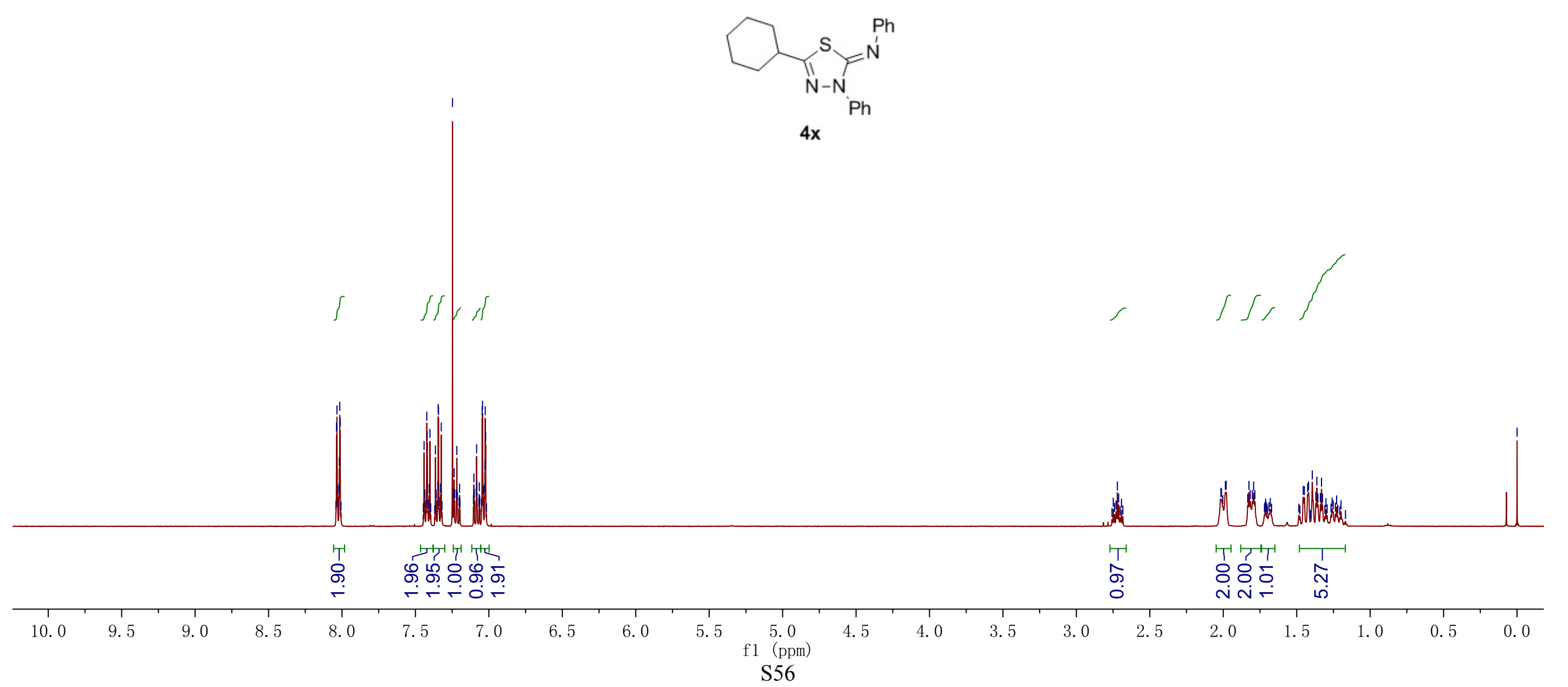


${ }^{13} \mathrm{C}$ NMR $\left(100 \mathrm{MHz}, \mathrm{CDCl}_{3}\right)$

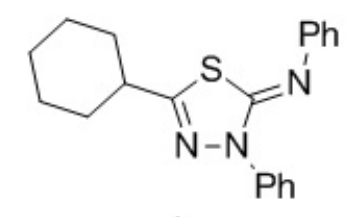

$4 x$

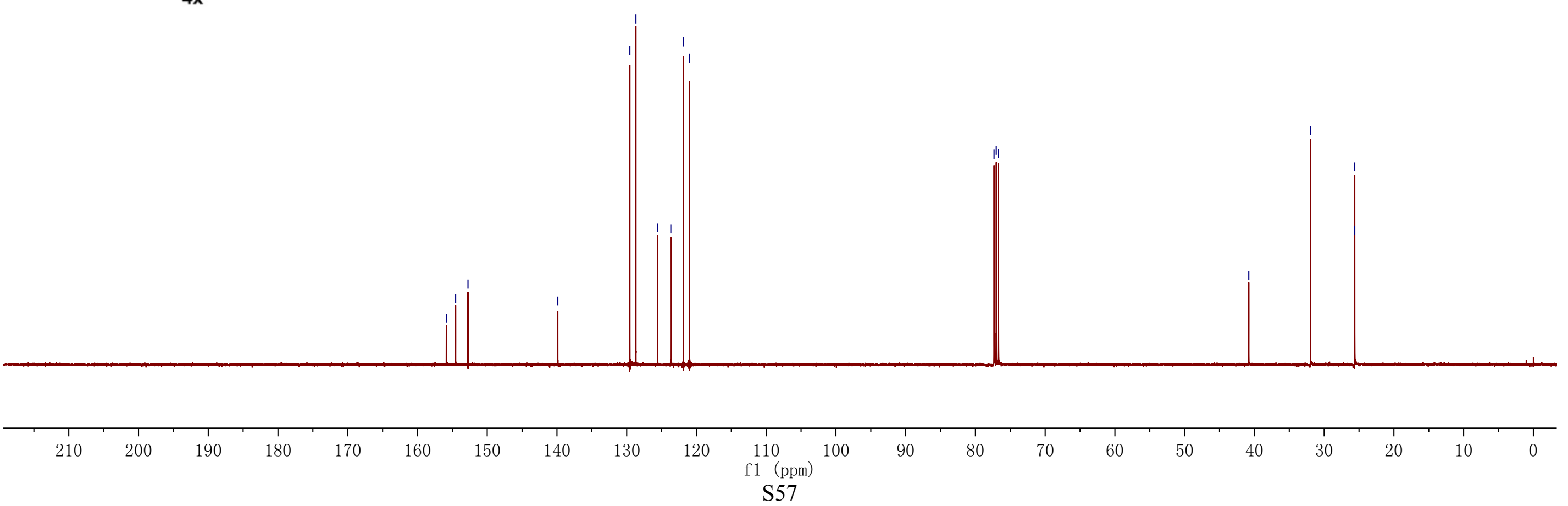


${ }^{1} \mathrm{H}$ NMR $\left(400 \mathrm{MHz}, \mathrm{CDCl}_{3}\right)$
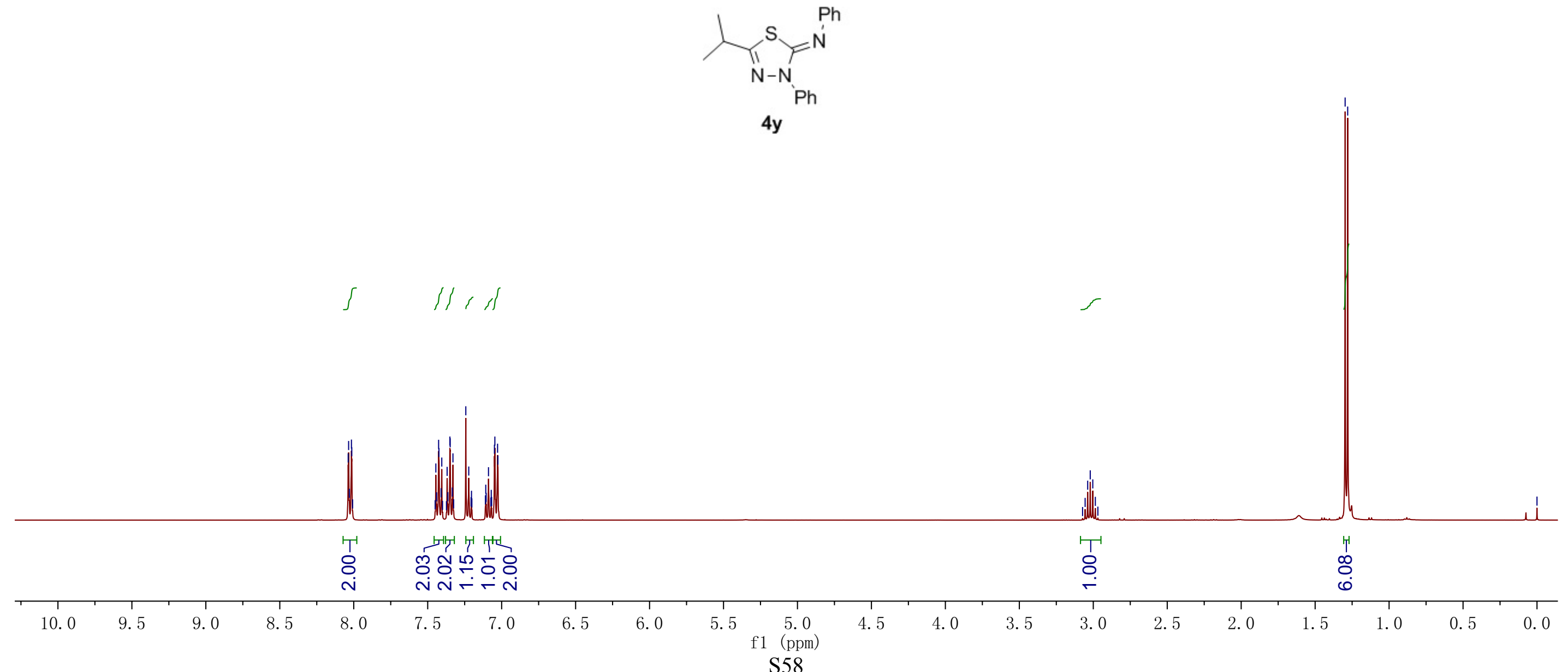
${ }^{13} \mathrm{C}$ NMR (100 MHz, $\left.\mathrm{CDCl}_{3}\right)$
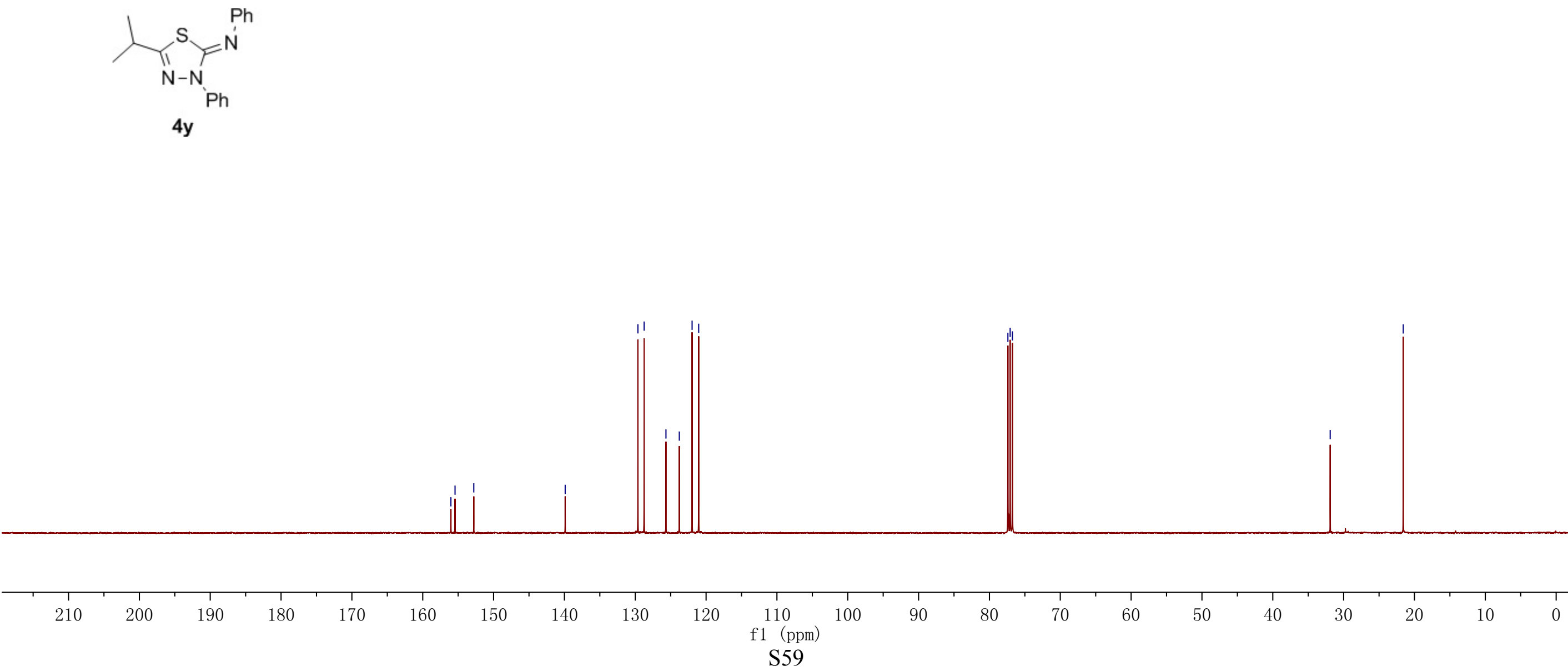
${ }^{1} \mathrm{H} \mathrm{NMR}\left(400 \mathrm{MHz}, \mathrm{CDCl}_{3}\right)$
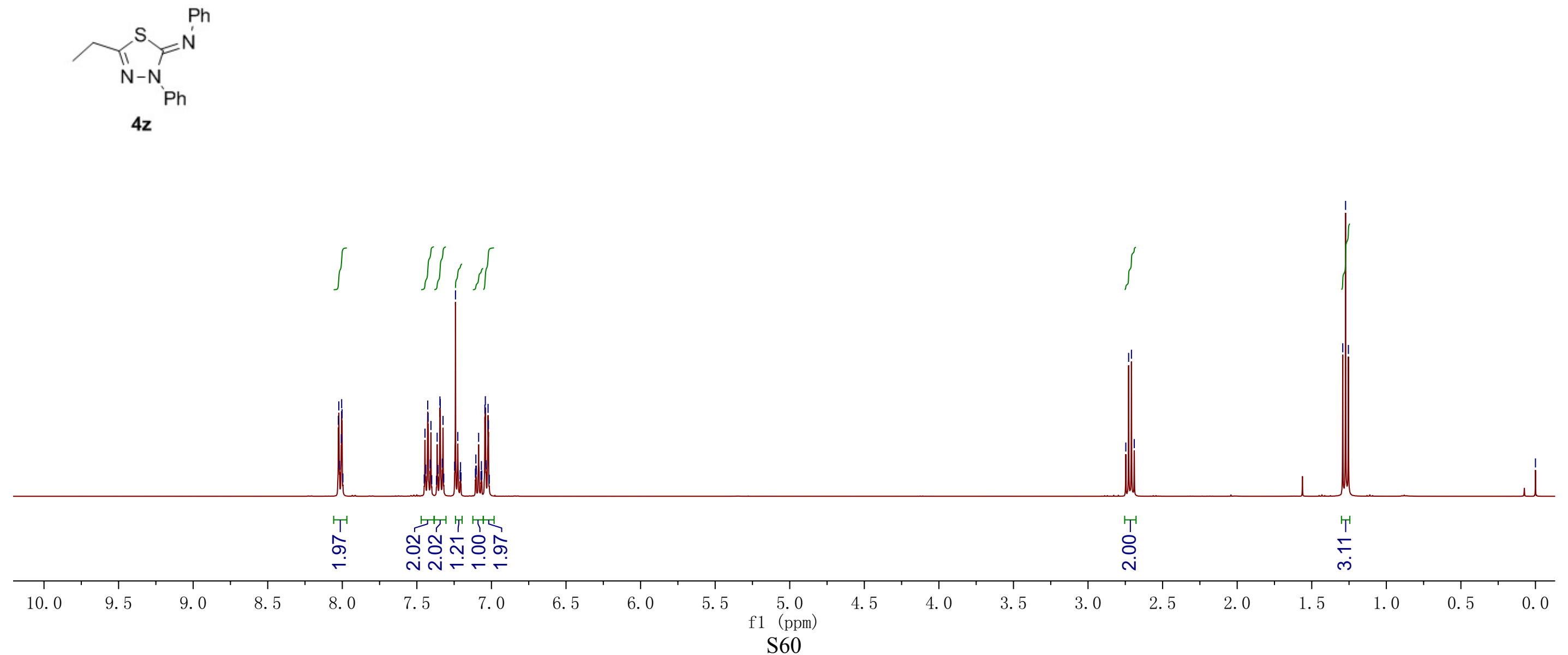
${ }^{13} \mathrm{C}$ NMR (100 MHz, $\left.\mathrm{CDCl}_{3}\right)$
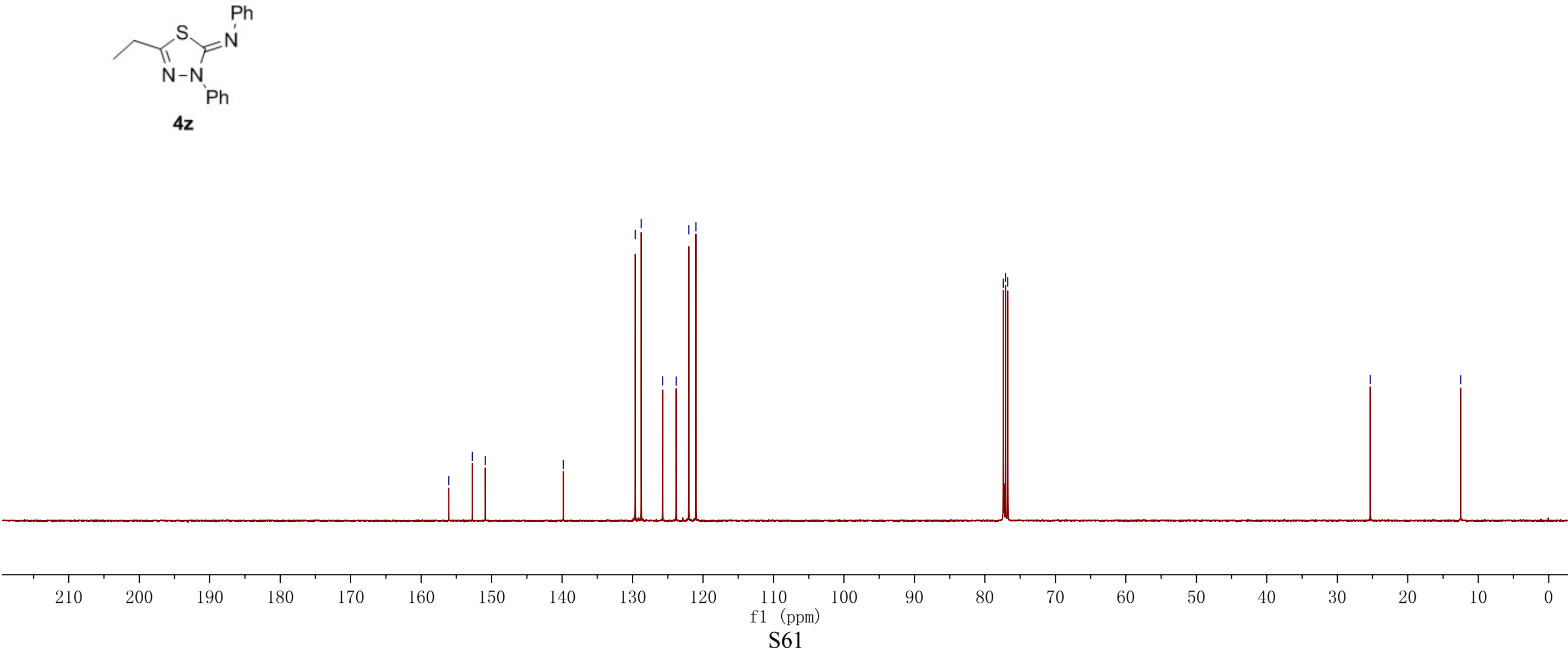
${ }^{1} \mathrm{H}$ NMR $\left(400 \mathrm{MHz}, \mathrm{CDCl}_{3}\right)$
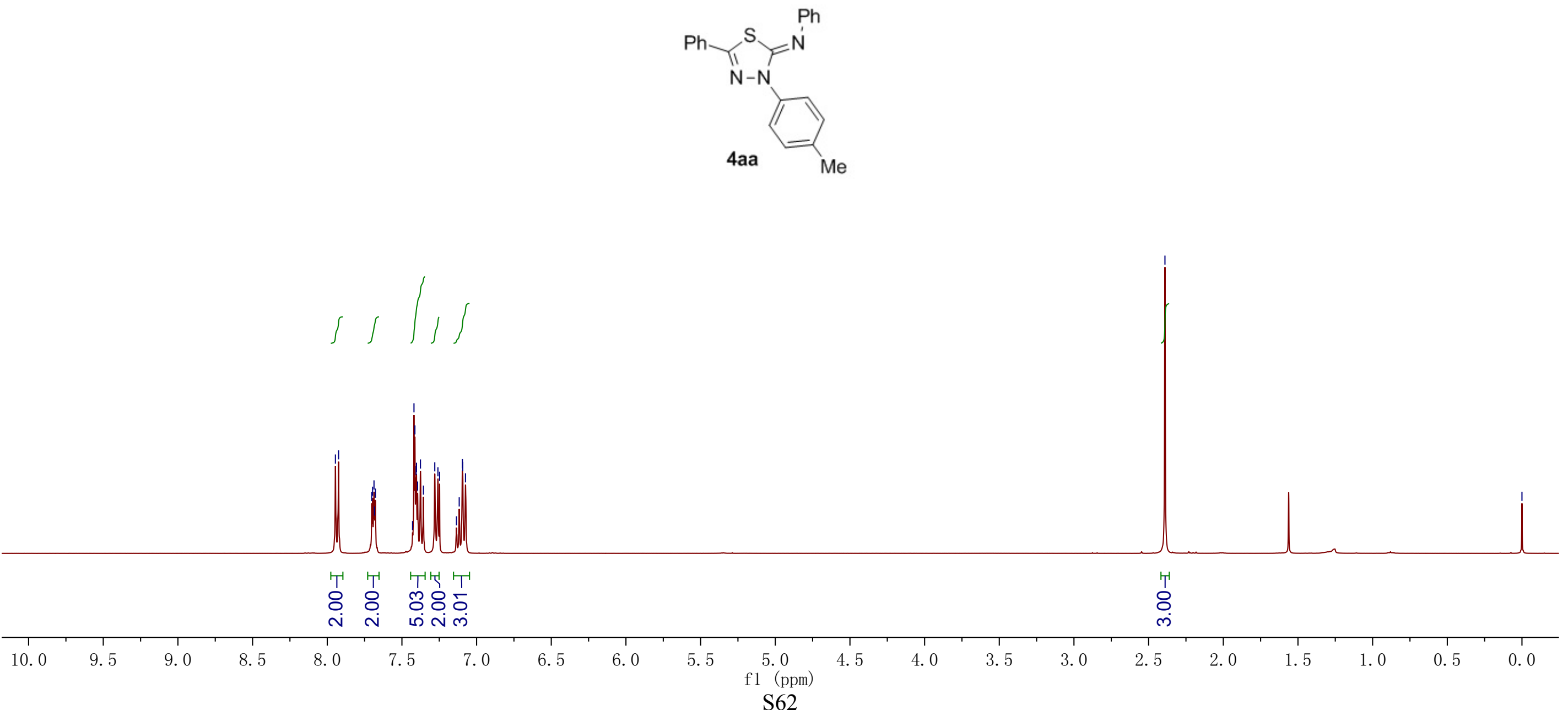
${ }^{13} \mathrm{C}$ NMR (100 MHz, $\left.\mathrm{CDCl}_{3}\right)$
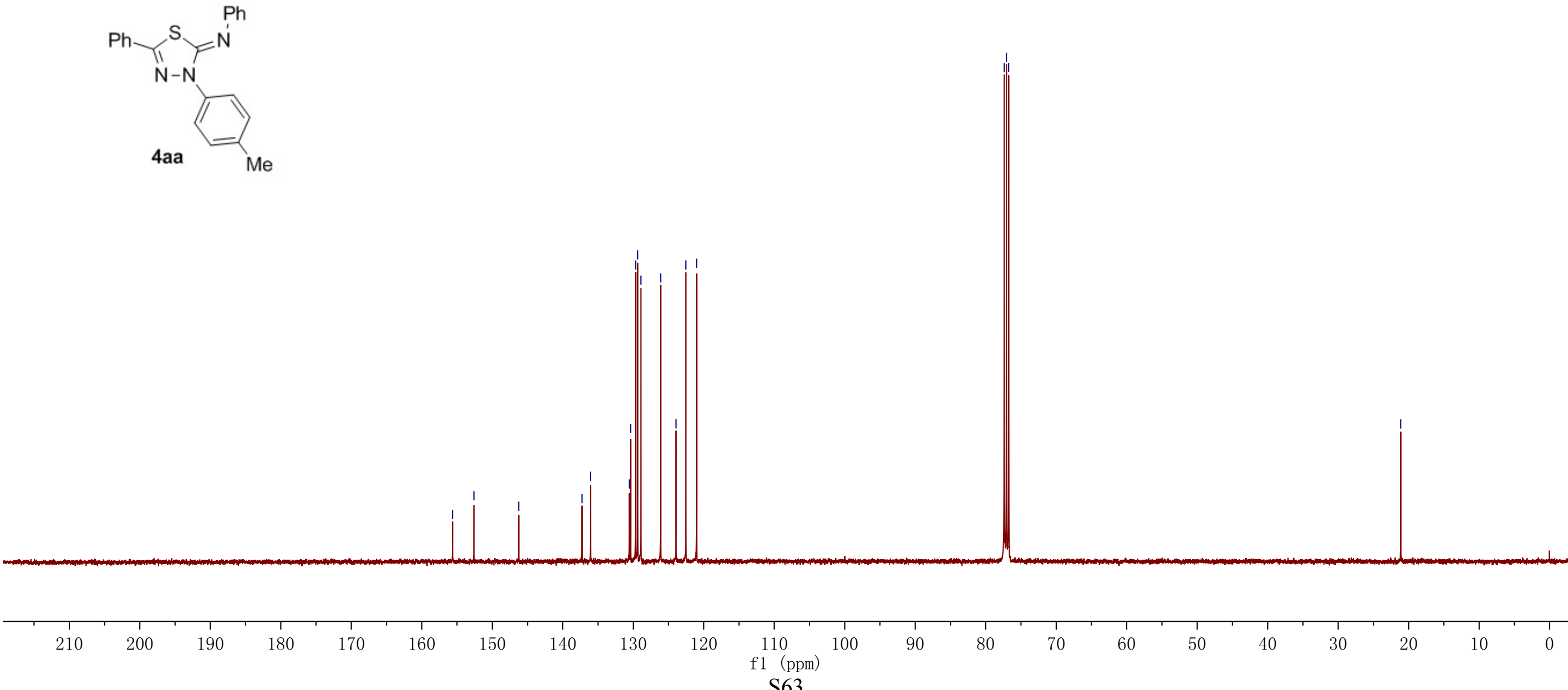
${ }^{1} \mathrm{H}$ NMR $\left(400 \mathrm{MHz}, \mathrm{CDCl}_{3}\right)$
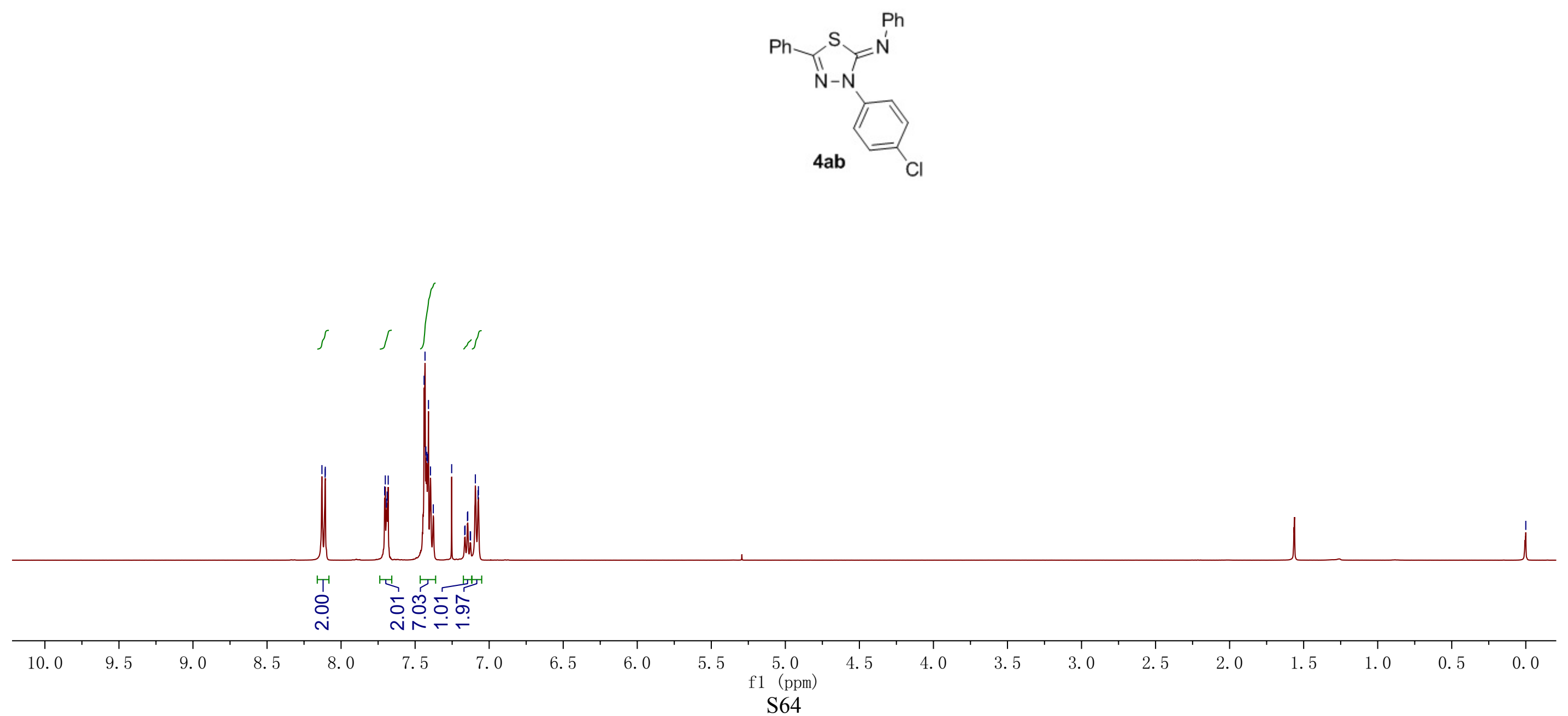
${ }^{13} \mathrm{C}$ NMR (100 MHz, $\left.\mathrm{CDCl}_{3}\right)$
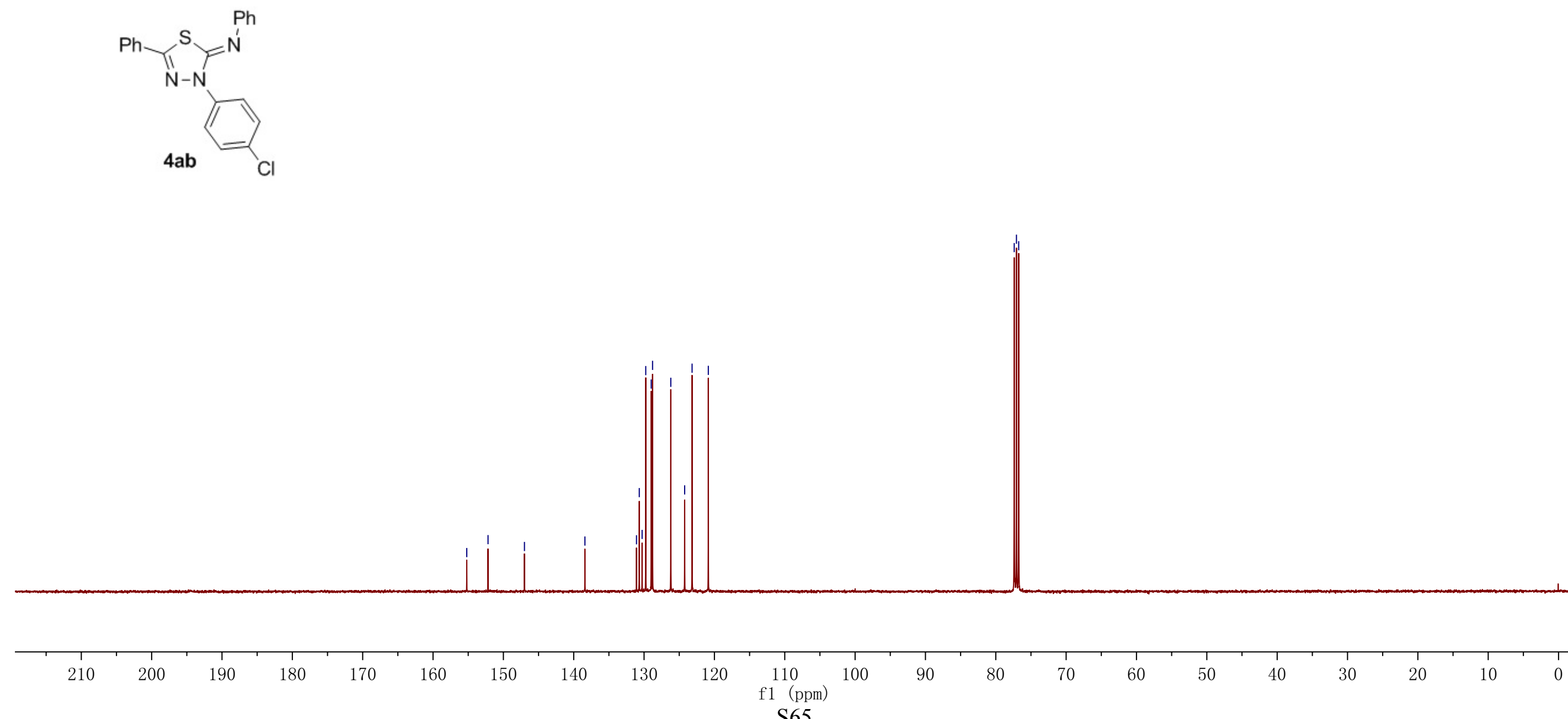
${ }^{1} \mathrm{H}$ NMR $\left(400 \mathrm{MHz}, \mathrm{CDCl}_{3}\right)$
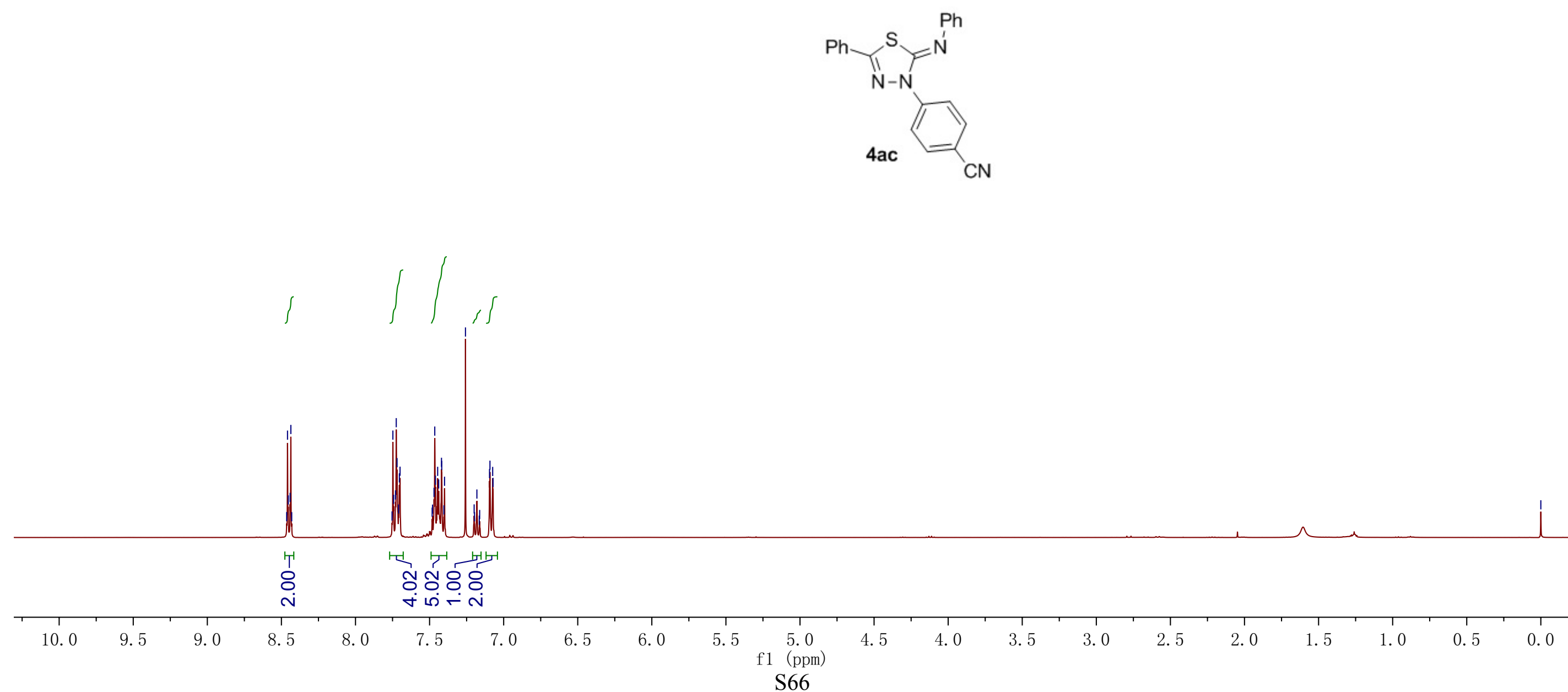
${ }^{13} \mathrm{C}$ NMR (100 MHz, $\left.\mathrm{CDCl}_{3}\right)$
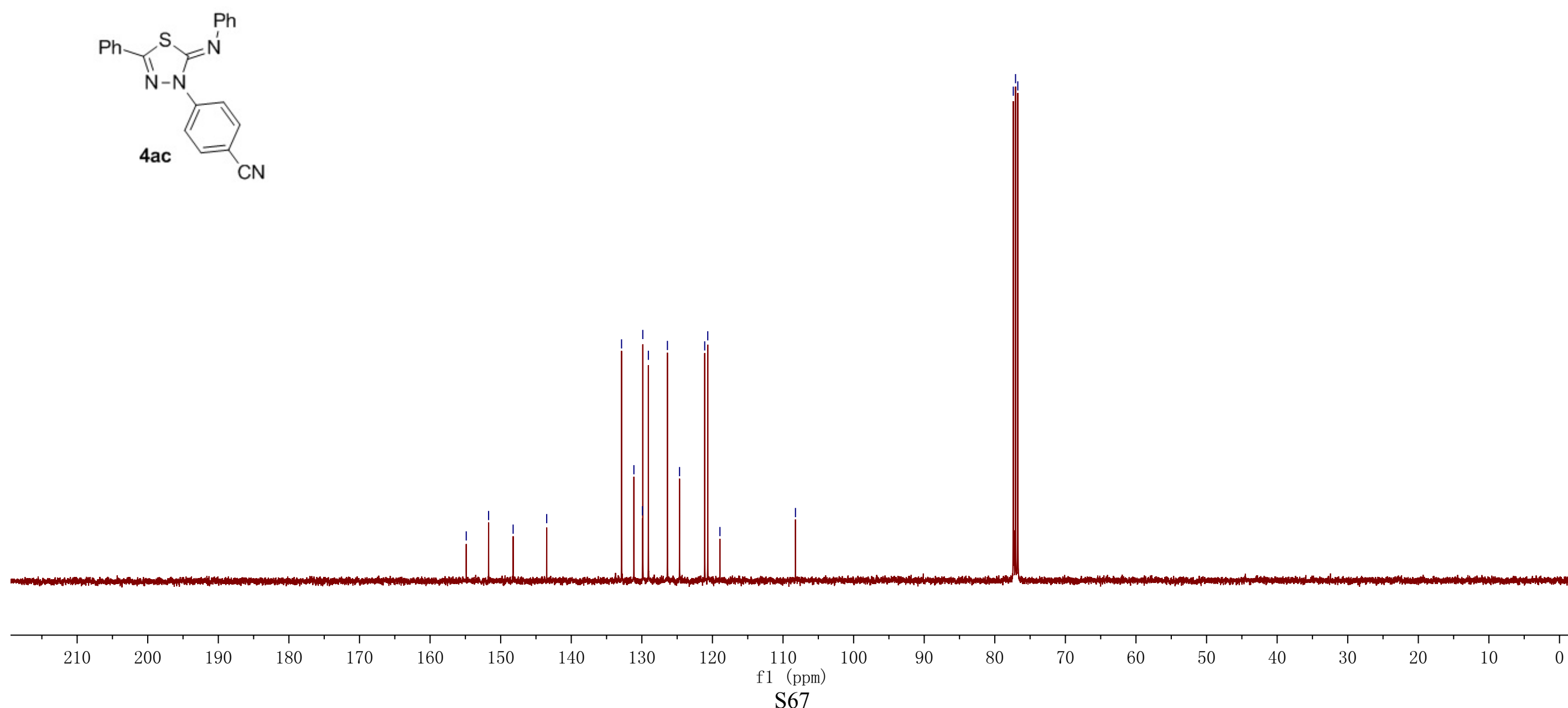
${ }^{1} \mathrm{H}$ NMR (400 MHz, $\left.\mathrm{CDCl}_{3}\right)$
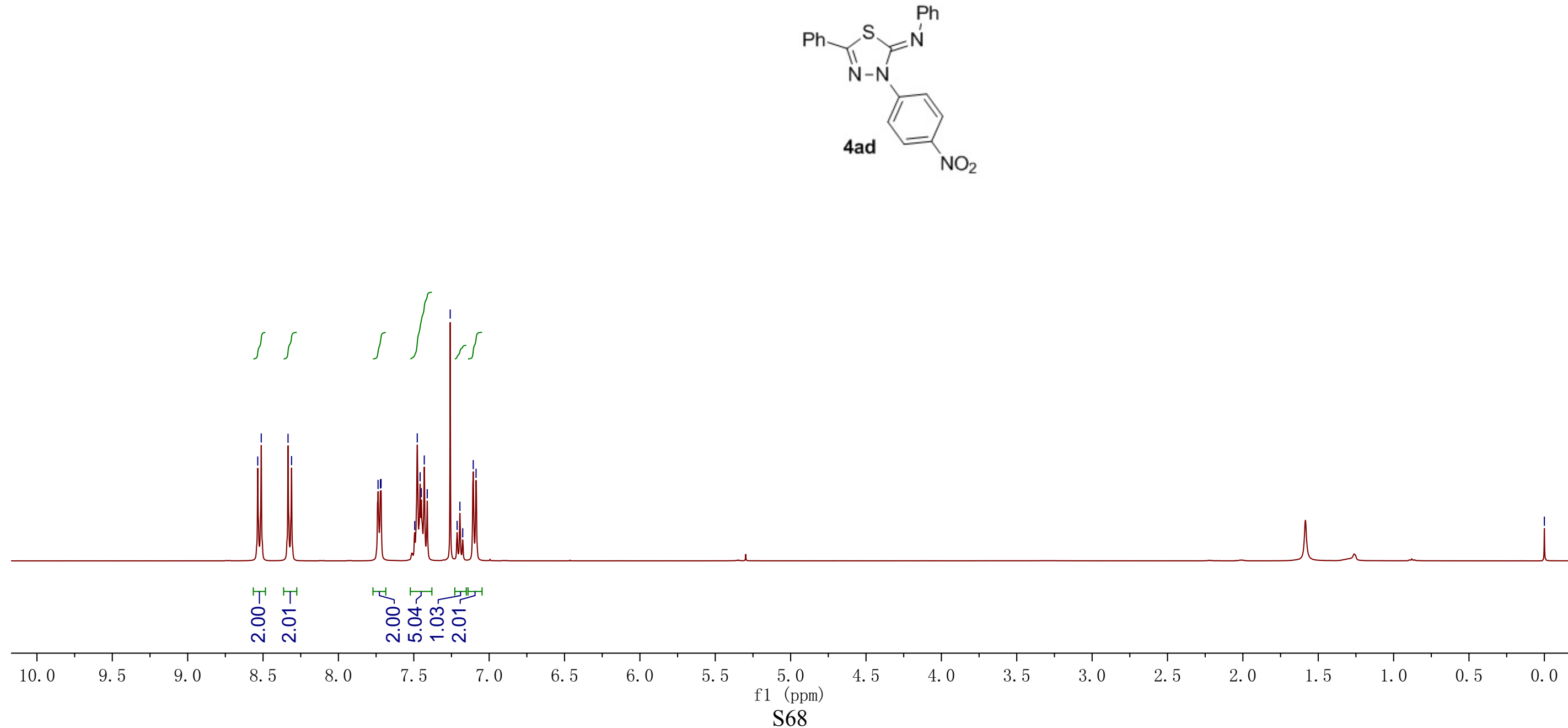
${ }^{13} \mathrm{C}$ NMR (100 MHz, $\left.\mathrm{CDCl}_{3}\right)$
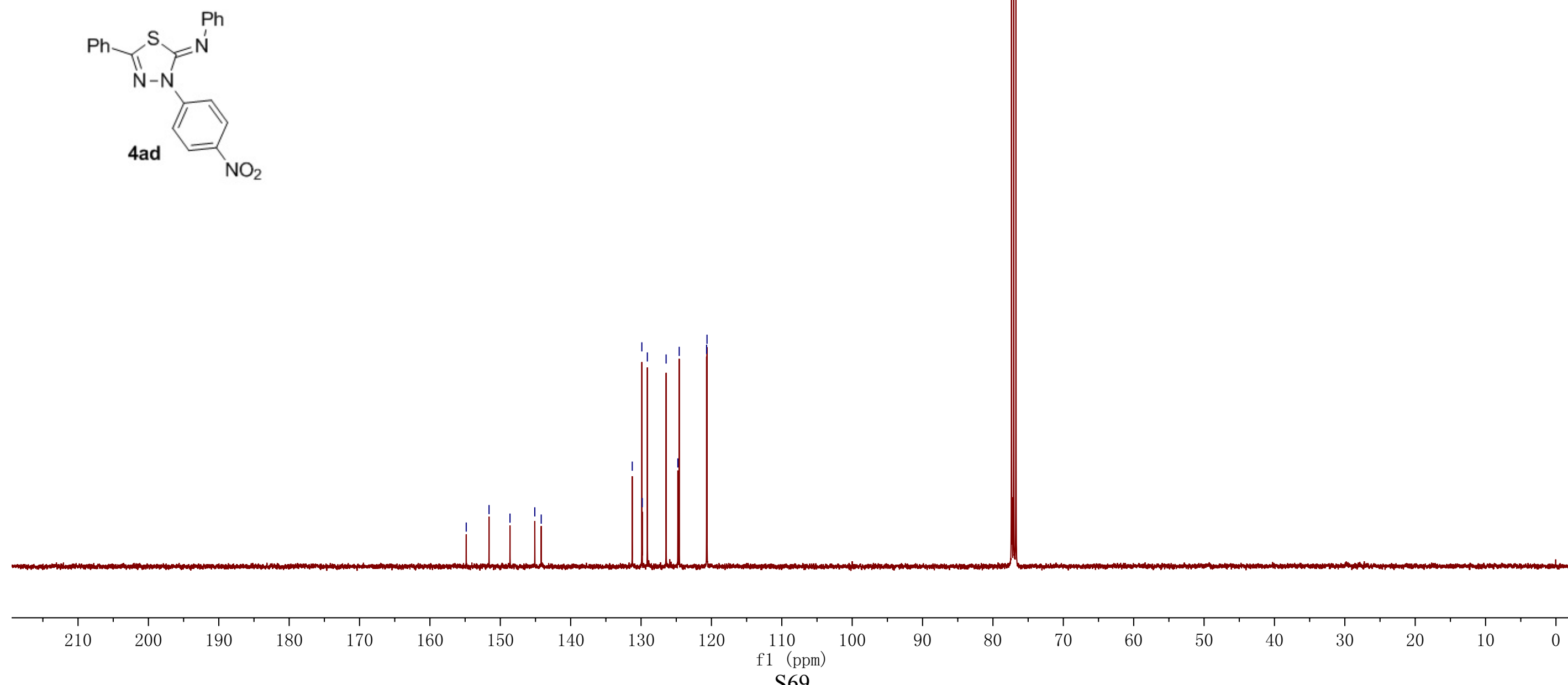
${ }^{1} \mathrm{H}$ NMR (400 MHz, $\mathrm{CDCl}_{3}$ )

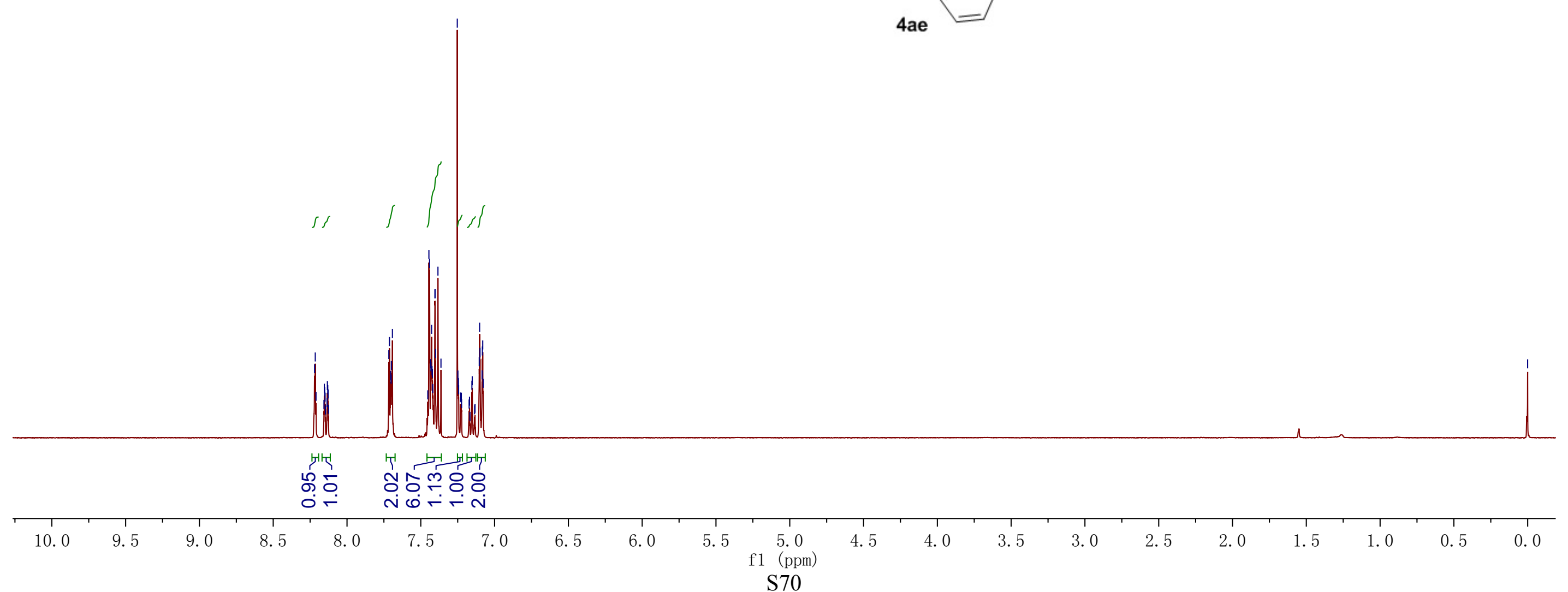


${ }^{13} \mathrm{C}$ NMR $\left(100 \mathrm{MHz}, \mathrm{CDCl}_{3}\right)$

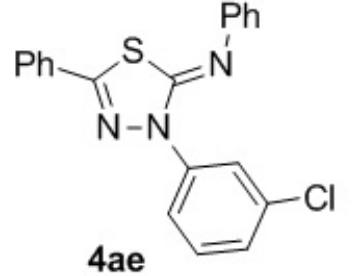

$4 \mathrm{ae}$
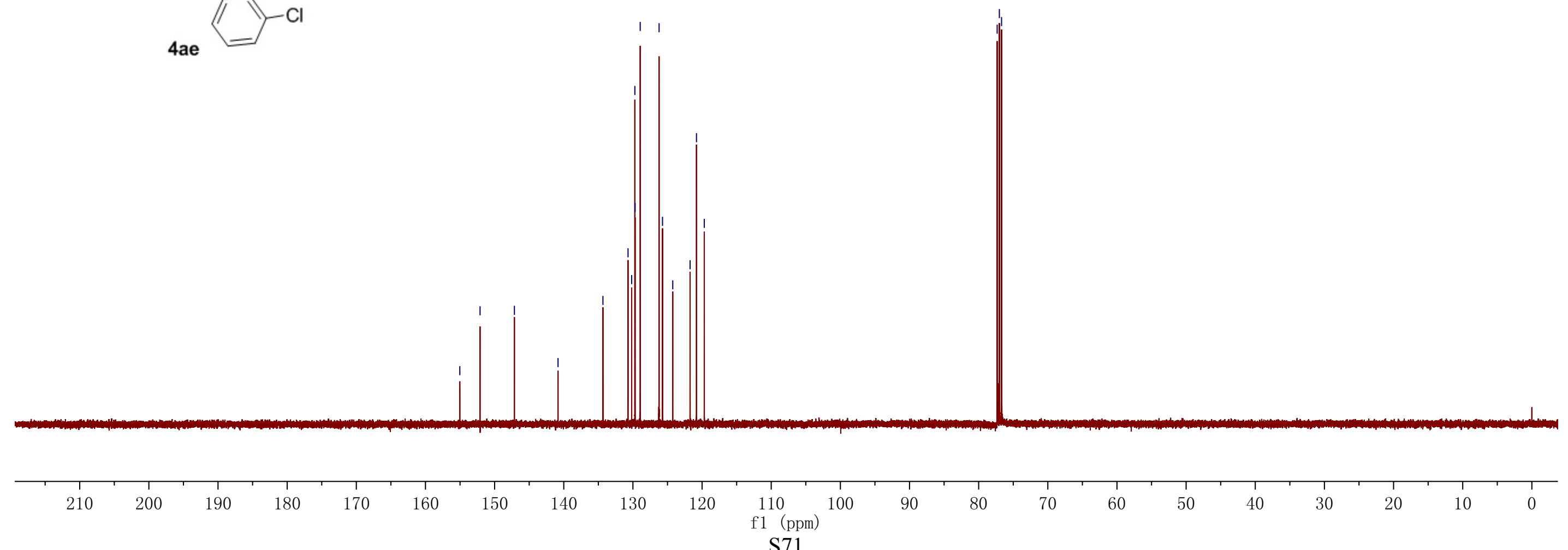
${ }^{1} \mathrm{H}$ NMR $\left(400 \mathrm{MHz}, \mathrm{CDCl}_{3}\right)$
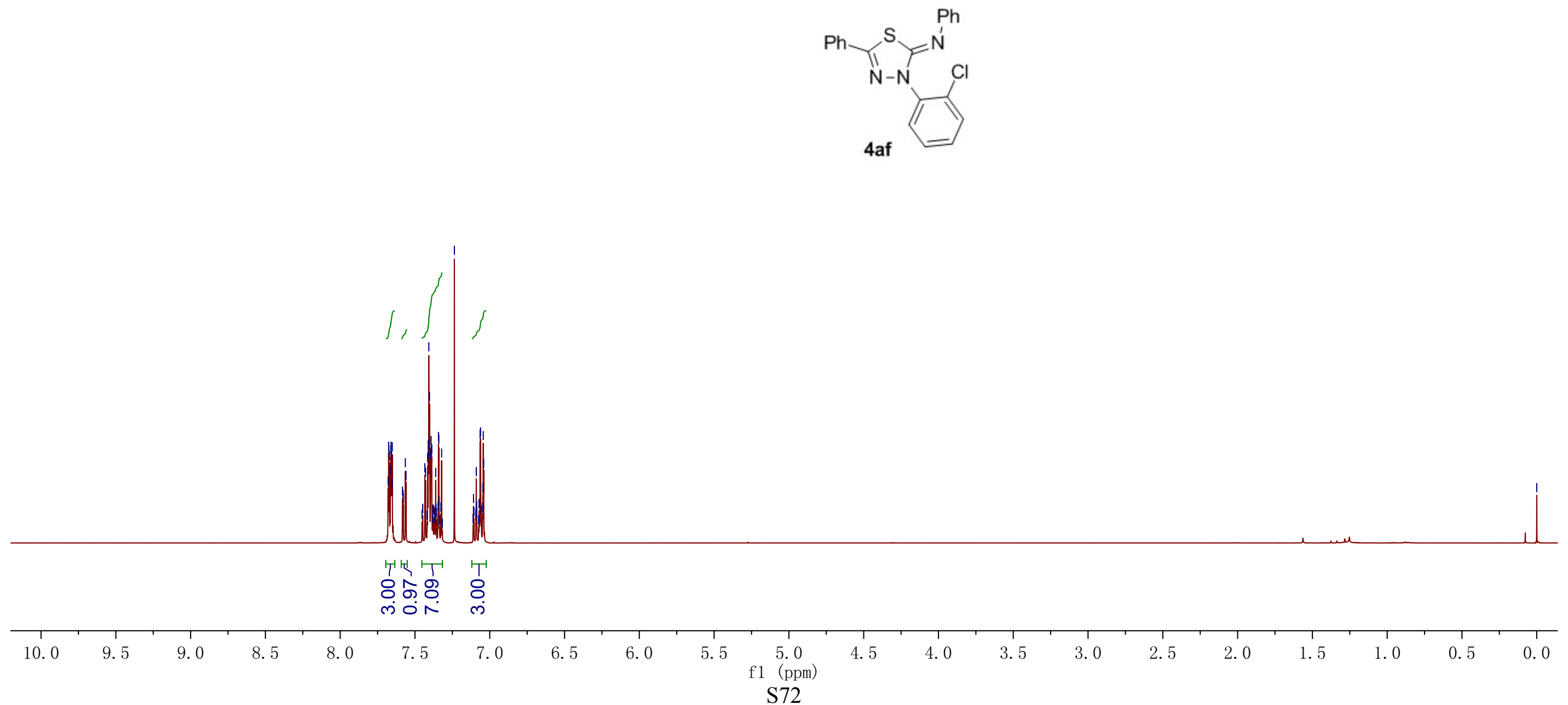
${ }^{13} \mathrm{C}$ NMR (100 MHz, $\left.\mathrm{CDCl}_{3}\right)$
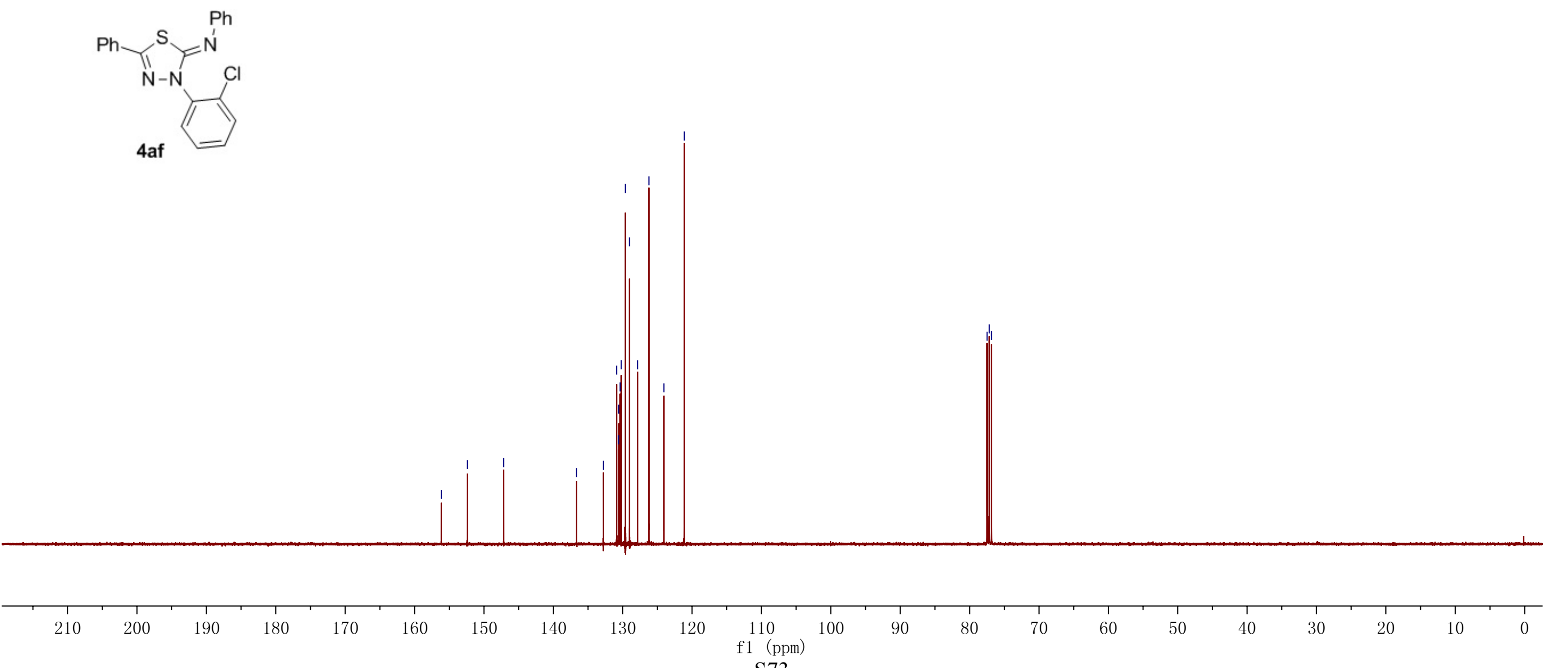
${ }^{1} \mathrm{H} \mathrm{NMR}\left(400 \mathrm{MHz}, \mathrm{CDCl}_{3}\right)$
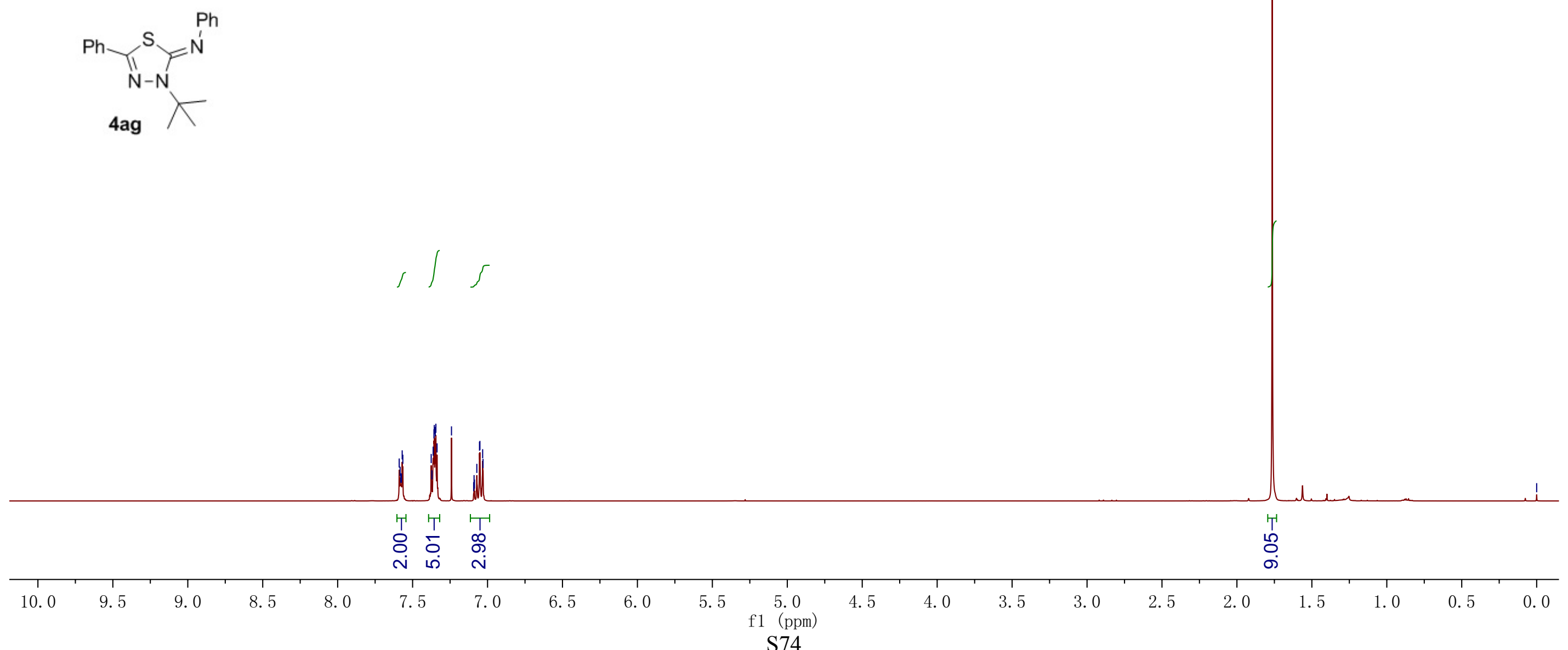
${ }^{13} \mathrm{C}$ NMR (100 MHz, $\left.\mathrm{CDCl}_{3}\right)$
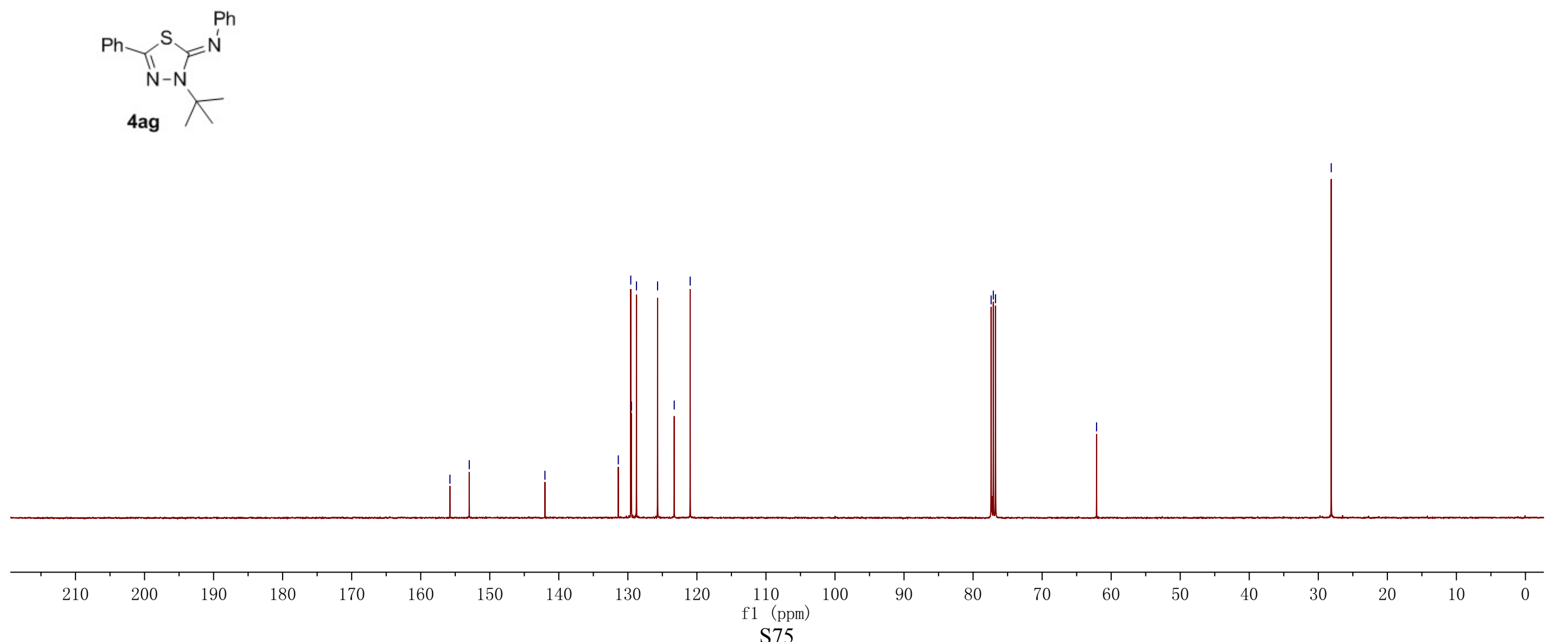